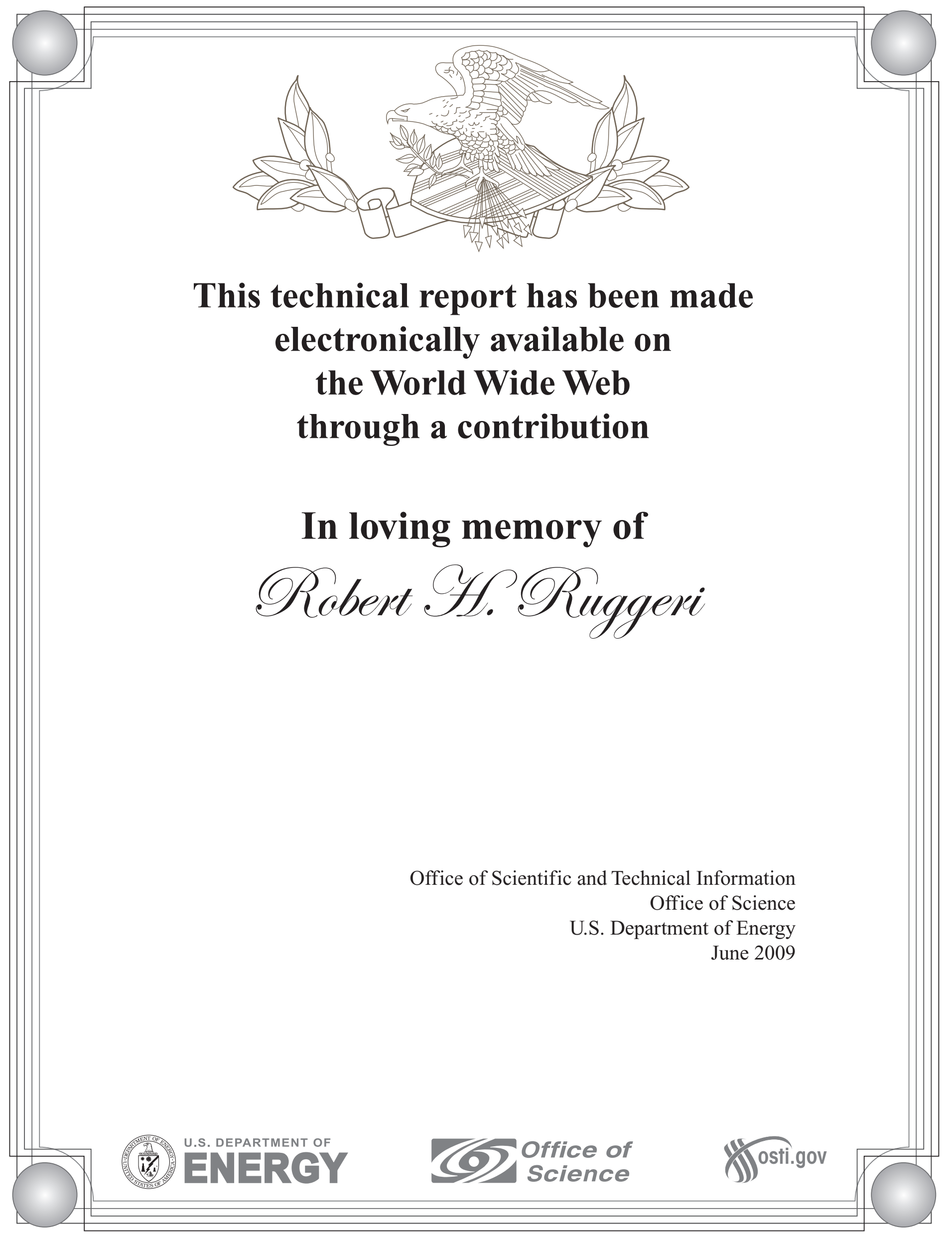


AUG 31964

UCRL-||4II

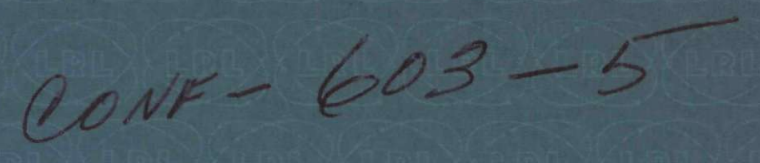

MGSTR

University of California

Ernest O. Lawrence
Radiation Laboratory

THE ELECTROMAGNETIC LEVITATION OF

Berkeley, California 


\section{DISCLAIMER}

This report was prepared as an account of work sponsored by an agency of the United States Government. Neither the United States Government nor any agency Thereof, nor any of their employees, makes any warranty, express or implied, or assumes any legal liability or responsibility for the accuracy, completeness, or usefulness of any information, apparatus, product, or process disclosed, or represents that its use would not infringe privately owned rights. Reference herein to any specific commercial product, process, or service by trade name, trademark, manufacturer, or otherwise does not necessarily constitute or imply its endorsement, recommendation, or favoring by the United States Government or any agency thereof. The views and opinions of authors expressed herein do not necessarily state or reflect those of the United States Government or any agency thereof. 


\section{DISCLAIMER}

Portions of this document may be illegible in electronic image products. Images are produced from the best available original document. 


\section{UNIVERSITY OF CALIFORNIA \\ Lawrence Radiation Laboratory \\ Berkeley, California}

AEC Contract No. W-7405-eng-48

THE ELECTROMAGNETIC LEVITATION OF METALS

Peter R. Rony

May 7, 1964 


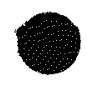

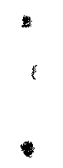


THE ELECTROMAGNETIC LEVITATION OF METALS

\section{Contents}

Abstract . . . . . . . . . . . . . . . . . . . . v

I. Introduction . . . . . . . . . . . . . . . . 1

II. Theory of the Electromagnetic Levitation of Solid Metals
A. Eddy Currents and Skin Depth.
2
B. Fundamental Levitation Equations. . . . . . . . . 8
C. Definition of $\mathrm{G}(\mathrm{x}), \mathrm{H}(\mathrm{x})$, and $\mathrm{F}(\mathrm{x}) \cdot$. . . . . . . 15
D. Effect of Coil Geometry . . . . . . . . . . . . 22

III. Application of the Levitation Equations

A. Simplified Levitation Equations . . . . . . . . . . 26

B. Typical Levitation Experiment . . . . . . . . . . 27

C. Levitation of Different Metals . . . . . . . . . . 32

D. Magnitude of the Magnetic Field . . . . . . . . . . 40

IV. Theory of the Electromagnetic Levitation of Liquid Metals. . . . 57

V. Future Developments
A. Improved Induction Heaters , . . . . . . . . . 62
B. Levitation of Dielectric Materials. . . . . . . . . . 65

VI. Summary and Conclusions . . . . . . . . . . . . . . 66

Appendix . . . . . . . . . . . . . . . . . . . 69

Notation . . . . . . . . . . . . . . . . . 83

Footnotes and References . . . . . . . . . . . . . . . . 86 


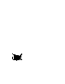

. 


\title{
THE ELECTROMAGNETIC LEVITATION OF METALS ${ }^{* \dagger}$
}

\author{
Peter R. Rony
}

\author{
Lawrence Radiation Laboratory and Department of Chemical Engineering \\ University of California \\ Berkeley, California \\ May 7, 1964
}

\begin{abstract}
A variety of subjects connected with the electromagnetic levitation of metals is considered. A complete theoretical description of the phenomenon is given, leading to the basic equations for the lifting force and power input for a metal sphere levitated in an inhomogeneous sinusoidally alternating magnetic field,
\end{abstract}

$$
\begin{aligned}
& \frac{\vec{F}}{W}=-\frac{3 G(x)}{2 \rho \mu_{0}}(\vec{B} \cdot \nabla) \vec{B} \\
& P=\frac{3 \pi R H(x)}{\sigma \mu_{0}^{2}}(\vec{B} \cdot \vec{B}),
\end{aligned}
$$

where

$$
x=R\left(\frac{1}{2} \omega \mu \sigma\right)^{1 / 2}
$$

$p, \sigma, \mu$, and $R$ are the metal density, electrical conductivity, permeability, and radius, respectively, $B$ is the magnetic-field strength, $\nabla$ is the vector del operator, and $\omega$ is the radian frequency of the magnetic field.

Both the force and power cannot be simultaneously controlled with the current-control knob of an induction heater. The suspended metal is suitably controlled either by operating at lower frequencies and supplying additional heat with an electron beam, plasma-arc torch, high-frequency induction heater, or reflection furnace, or by operating at high frequencies and employing high-thermal-conductivity gases such as hydrogen and helium to cool the metal. 
Values indicating the ease of levitation of $1-\mathrm{cm}$-radius spheres of more than 50 different metals at $0^{\circ} \mathrm{C}$ and at their respective melting points are given. When frequencies in the 10 to $100 \mathrm{kc} / \mathrm{sec}$ range are used, the density of the metal chiefly determines the ease of levitation. Refractory, semiconducting, or dielectric materials can be levitated either by heating them to high temperatures to decrease their electrical resistivity or by coating a light and inert metal of high melting point with the material to be studied.

Liquid sodium surrounded by a mineral oil of almost identical density can be used to study the magnetic-field distribution of levitation coils. Liquid mercury can be used to determine the ability of levitation coils to suspend high-temperature liquid metals.

Although the use of this technique with large commercial quantities of liquid metals appears discouraging, the levitation of long rods or plane sheets of liquid metals does appea to be feasible, provided that these configurations are not dynamically unstable.

More attention should be given to the improvement of commercial levitation equipment through the use of variable frequencies, solid-state circuits, and high-permeability magnetic core materials with low losses. The ideal levitation power supply would have a variable frequency for the range

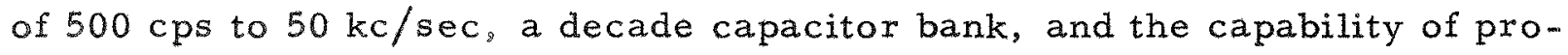
ducing a square or sine-wave alternating magnetic field up to several kilogauss over a $75 \mathrm{~cm}^{3}$ volume. 


\section{INTRODUCTION}

Levitation, the phenomenon of suspending a dense object without support, was first confirmed both theoretically and experimentally for metals in the liquid and solid states by the Westinghouse group of Okress, Wroughton, Comenetz, Brace, and Kelly early in 1950. In the succeeding decade, the use of this technique has spread to numerous areas of metallurgy. Two levitation melting furnaces have become commercially available ${ }^{1,2}$ and. other induction heaters have been adapted for such work. 3,4 Demonstrated applications have included alloy preparation, $1,5,6$ metal purification, 7 vapor plating, ${ }^{8}$ sintering, 9 determination of liquid-metal densities and emissivities, ${ }^{6,10,11}$ physicochemical studies of gas-metal reactions, ${ }^{12,13}$ metal supercooling, ${ }^{6}$ vaporization phenomena, ${ }^{14,15}$ and alloy thermodynamics. 16 Despite such advances, the levitation of liquid metals has often been considered to be an art. Furthermore, the technique itself has never fulfilled its optimistically stated earlier promise, ${ }^{\prime \prime}$ the full-scale [ commercial] production.. [of] quantities of the world"s purest metals and some of its finest alloys. 5

The driving force for this study is the great potential of electromagnetic levitation of metals as a tool in vacuum metallurgy, provided its assets and limitations are properly understood and appreciated. Since the metallurgist should have more than a casual knowledge of electromagnetic theory as applied to metal levitation, the derivation of the fundamental levitation equations is given in full detail in the appendix. In Sec. II it is shown that the fundamental equations are valid and do allow the quantitative prediction of the lifting force on and the power input to a levitated metal sample. Section III discusses the implications of these equations and Secs. IV and V point to future directions for both research on and practical applications of this technique. 
The symbols used in this paper are defined in a section (Notations) that follows the Appendix.

\section{HEORY OE ELECTROMAGNETIC LEVITATION OF SOLID METALS}

\section{A. Eddy Jurrents and Skin Depth}

The study of electromagnetic levitation is simply the determination of the forces that time-varying magnetic fields exert on any conducting material. This conducting material may be a metal, a semiconductor or insulator that has been heated to a high temperature to increase its electrical conductivity, a gaseous plasma, an electrolytic solution, or a molten electrolyte. The latter two systems usually do not have electrical conductivities high enough for the fields to have much of an effect.

What happens when a conducting material is placed in a time-varying magnetic field? To answer this question, consider a homogeneous metal sphere of electrical concuctivity $\sigma$, magnetic permeability $\mu$, and radius $R$ surrounded by a vacuum in which there exists a uniform $z$-directed sinusoidally alternating magnetic field of frequency $\omega$, as shown in Fig. 1. The application of Maxwell's equations and the proper boundary conditions to this system 17,18 shows that sinusoidally alternating circular currents, all of the same frequency, are induced in the metal sphere. These currents, commonly called eddy currents, are illustrated in Fig. 2. They have a maximum value at the equator of the sphere but vanish at the poles. Their magnitude, phase, and the extent to which they penetrate into the metal sphere depend upon $\sigma, \mu, R$, and the frequency and magnitude of the applied magnetic field.

For a quick estimate of the effect of an alternating magnetic field on a metal, it is convenient to define a quantity called the skin depth, which is given by the formula 


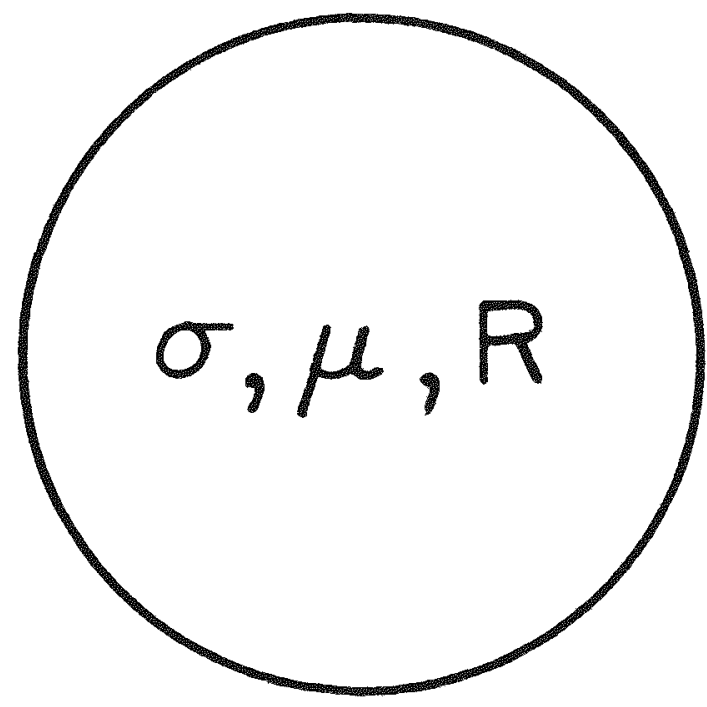

$\underset{m}{B}=B e^{j \omega \dagger} \vec{e}_{z}$
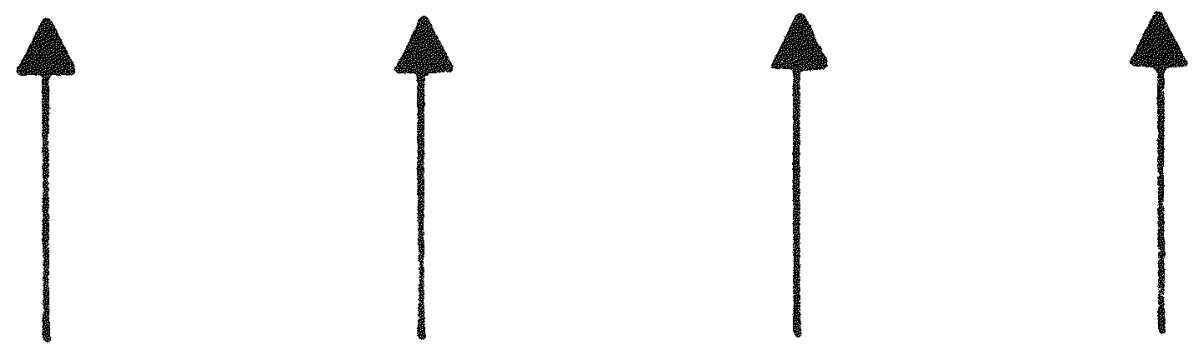

MUB -3033

Fig. 1. Homogeneous metal sphere of electrical conductivity $\sigma$, permeability $\mu$, and radius $R$ in a uniform $z$-directed sinusoidally alternating magnetic field. 


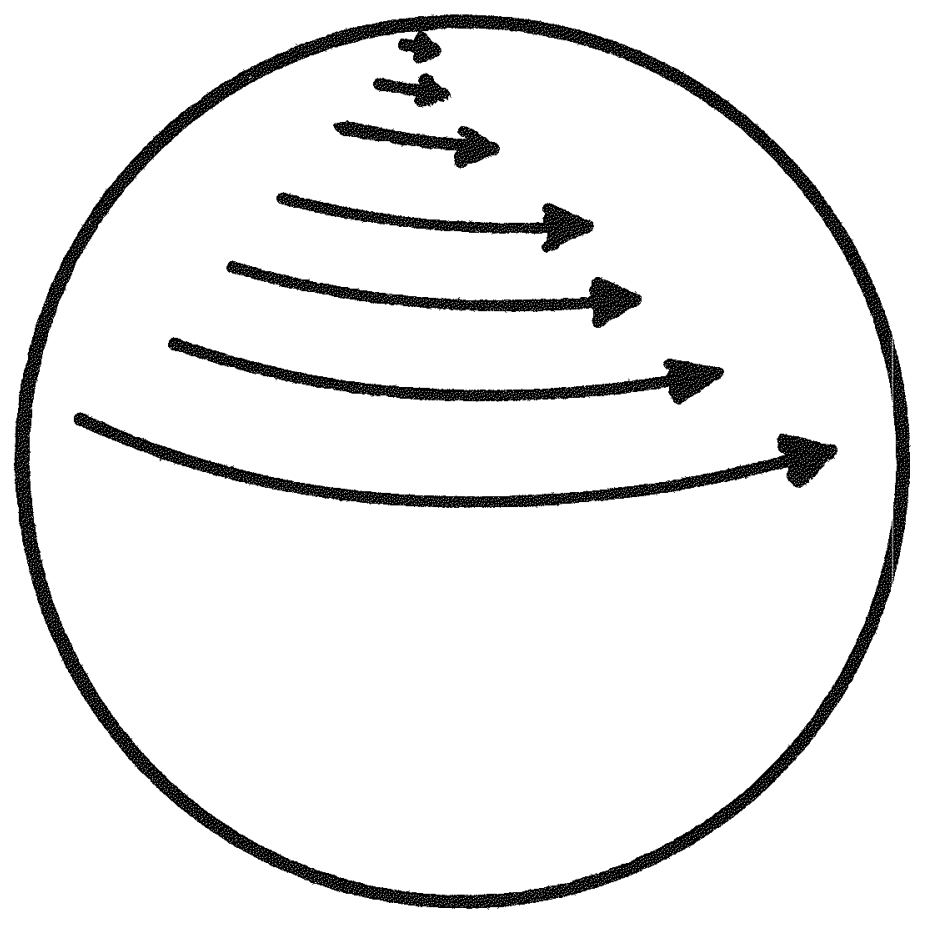

MUB -3015

Fig. 2. Eddy currents induced in the metal sphere. 


$$
\delta=\left(\frac{1}{2} \omega \mu \sigma\right)^{-1 / 2}
$$

This quantity appears quite naturally in solutions describing eddy currents derived from the appropriate Maxwell equations. As the skin depth is an important parameter, several conclusions can be made from a knowledge of its value alone.

In levitation and induction-heating applications, the effect of the eddy currents induced in the conductor is deliberately maximized. For other applications, such as in transformers, the presence of eddy currents constitutes a power loss and is thus quite undesirable; in such cases, the iron core is broken into thin insulated layers to minimize the effect of these currents. If the frequencies are so large that even this solution is unsatisfactory, highpermeability low-conductivity materials known as ferrites are used for the transformer cores.

There is another fundamental distinction between levitation and induction heating. In levitation, two variables must be controlled: the force on the metal sample and the power input to the metal. In induction heating, only the power input to the workpiece must be controlled. Whereas one knob on the induction heater, the current-control knob, suffices for induction-heating applications, it is not sufficient for levitation work. This point will be considered in a later section.

Physically, the skin depth is that surface thickness of a conductor whose dc resistance is equivalent to the total ac resistance. This definition, involving the resistance of a fictitious surface layer, is somewhat after-the. fact. It has been found to be a convenient descrpiton for an otherwise obscure collection of parameters. 
The skin depth is also commonly defined as that depth below the surface of a conductor at which the current density or magnetic field has decreased to $1 / \mathrm{e}$, or $37 \%$, of its value at the surface (Fig. 3). Beyond about three or four skin depths into the conductor, these two quantities have essentially disappeared. At high frequencies, neither an alternating magnetic field nor the induced eddy currents exist in the interior of a conducting material. The induced eddy currents reach a limiting value and, by any reasonable description, can be considered as surface currents, which explains the use of hollow metals for carrying high-frequency currents. The interior metal region is essentially superfluous and can be easily removed to conserve costly metal and to permit cooling by a circulating fluid such as water.

Once the concept of eddy currents has been grasped, the difference between levitation and induction heating can be understood. In levitation, ad.vantage is taken of the force of repulsion between the induced eddy currents and the time-varying current in the conductors producing the magnetic field, or, alternatively, the force of repulsion between the applied and eddy-current alternating magnetic fields. The dissipation of energy inside the conducling material occurs simultaneously, but this can be controlled generally by operating at the correct frequency. In induction heating, the importance of these physical effects is reversed and advantage is mainly taken of the heat dissipated by the eddy currents. By operating at high frequencies and moderate field strengths, a conducting material can be heated but not levitated. Thus, the two effects, force and heating, are somewhat independent of each other. For best results, one or the other should be maximized for a particular application。 


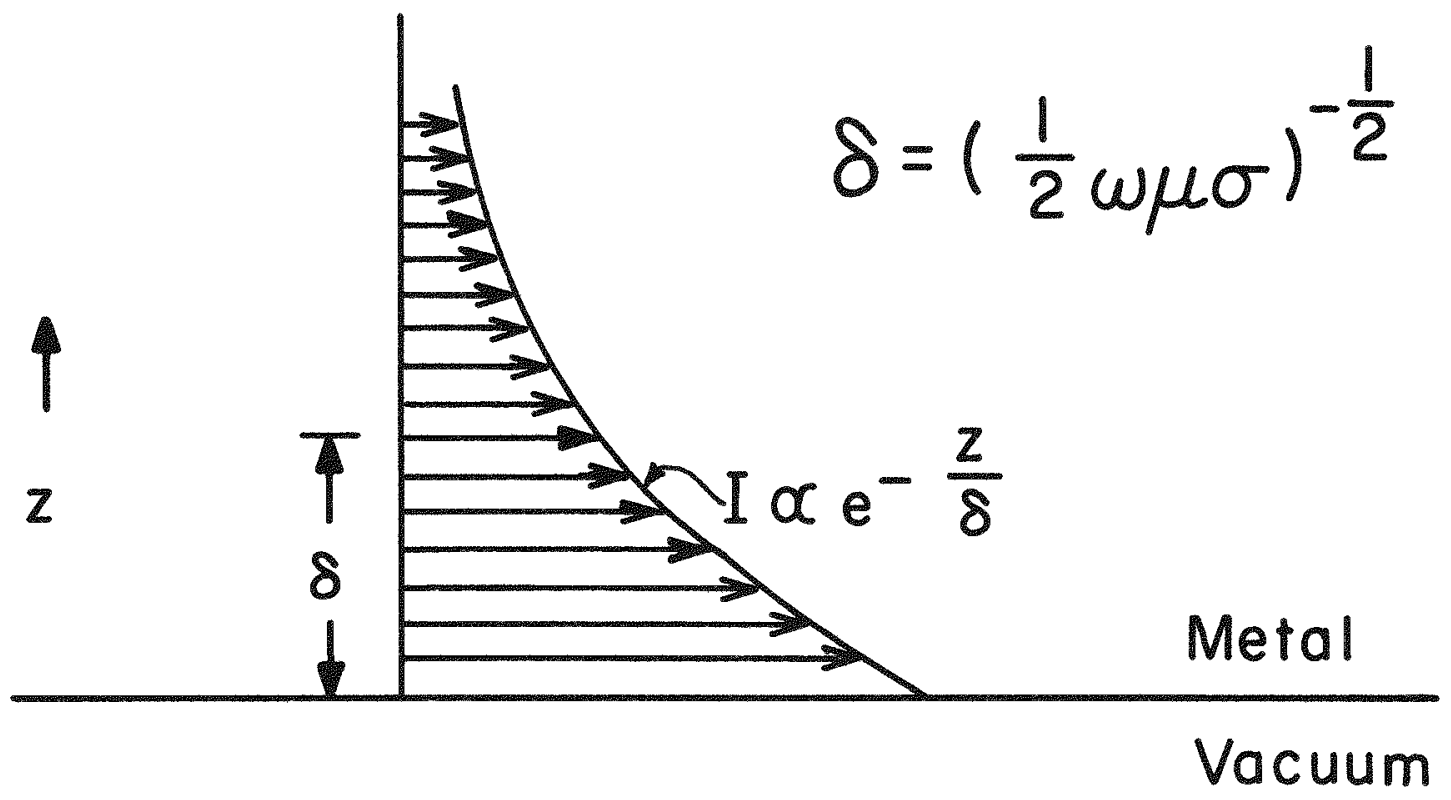

MUB -3016

Fig. 3. Skin depth for semi-infinite plane conductor. 


\section{B. Fundamental Levitation Equations}

The system shown in Fig. 1, a metal sphere of conductivity $\sigma$, permeability $\mu$, and radius $R$ located in a uniform unidirectional alternating magnetic field of frequency $\omega$, is precisely the one suggested by R.C. Mason ${ }^{19}$ and first used by Okress et al. ${ }^{19}$ to develop the fundamental equations of levitation theory. The detailed mathematical derivation is included in the appendix. The fundamental result of the calculations is that the metal sphere behaves exactly as an idealized magnetic dipole loop. For every metal sphere located in a uniform alternating magnetic field $\vec{B}$ a magnetic dipole moment $\vec{M}$ can be rigorously defined. The equivalence of the magnetic-field distributions for an idealized magnetic-dipole loop and a metal sphere with induced eddy currents is illustrated in Fig. 4. As a consequence of this result, the total force $\vec{F}$ acting on a rigid metal sphere is given by the relatively simple formula

$$
\vec{F}=(\vec{M} \cdot \nabla) \vec{B}
$$

where $\nabla$ is the vector del operator, which in cylindrical coordinates is defined as

$$
\nabla=\vec{e}_{r} \frac{\partial}{\partial r}+\frac{\vec{e}_{\theta}}{r} \frac{\partial}{\partial \theta}+\vec{e}_{z} \frac{\partial}{\partial z}
$$

An important simplification is made in deriving formula (2). This has been best stated by Okress et al.," "If now the sphere is set into a non-uniform alternating field, then to a first approximation the force may be calculated by replacing the sphere by the dipole that would be produced by a fictitious uniform alternating field having everywhere the value the given non-uniform field has at the center of the sphere. The actual non-uniform field would exert a force upon this dipole." If this simplification could not be made, the calculation of the total force upon the metal sphere would be prohibitively difficult. 

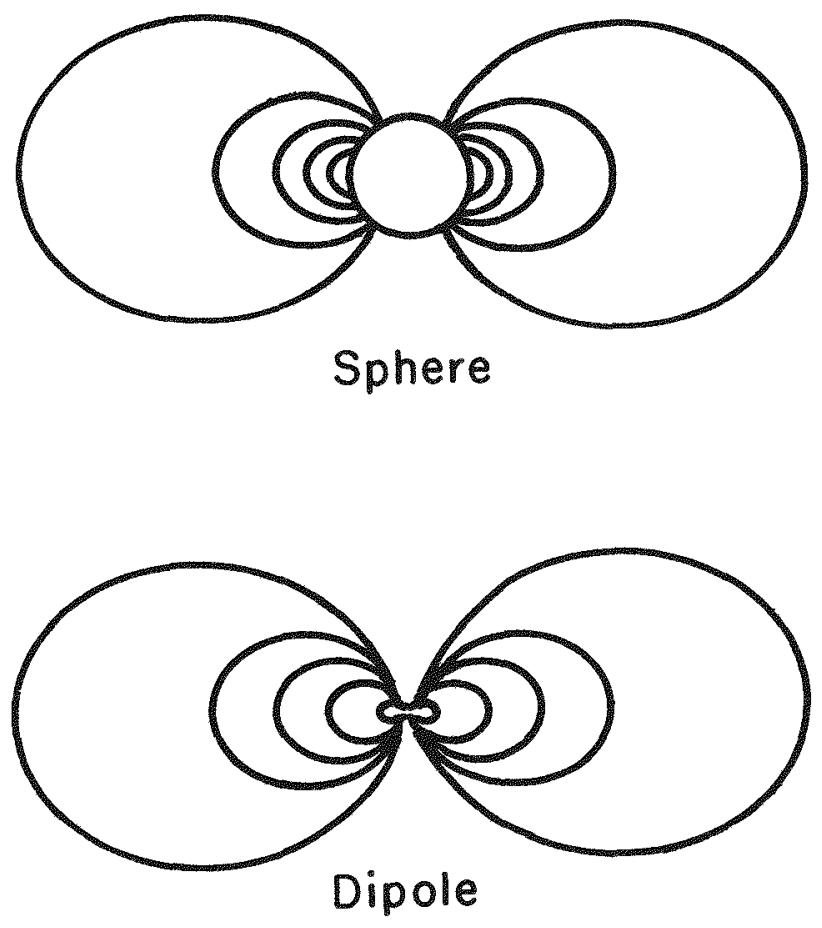

$M U B-3017$

Fig. 4. Lines of force for (a) a metal sphere with induced eddy currents and (b) a magnetic dipole. 
The complete equations for the force-to-weight ratio for the metal sphere and the power absorbed by it, as given in the appendix, are

$$
\begin{aligned}
& \frac{\vec{E}}{W}=-\frac{3 G(x)}{2 \rho \mu_{0}}(\vec{B} \cdot \nabla) \vec{B} \\
& P=\frac{3 \pi R H(x)}{\sigma \mu_{0}^{2}}(\vec{B} \cdot \vec{B}),
\end{aligned}
$$

where the parameter $\mathrm{x}$ is defined as the ratio of the metal sphere radius to the skin depth

$$
\mathrm{x}=\frac{\mathrm{R}}{\delta}
$$

and $G(x)$ and $H(x)$ are specified functions of this quantity。

The approximation used in deriving these equations is most valid for extremely small spheres and relatively small magnetic-field gradients.

Okress et al., however, have experimentally demonstrated that the theoretical result given in Eq. (3) is valid to a high degree of accuracy for 1 -in. brass balls suspended in 4.85 -in. copper coils using 600 amperes at $9.6 \mathrm{kc} / \mathrm{sec}$ and a Jolly balance. ${ }^{19}$ Their findings are verified by the coincidence of their theoretical and experimental curves for the coil configurations shown in Figs. 5,6, and 7. Theoretical calculations were not made for the configuration shown in Fig. 8. Since levitation calculations do not demand three or fourfigure accuracy: the above equations are more than sufficient for most cases.

Formulas (3) and (4) can be rewritten in the following manner

$$
\begin{aligned}
& \text { Force }=\left(\begin{array}{c}
\text { coil-geometry } \\
\text { Wunction }
\end{array}\right)\left(\begin{array}{c}
\text { skin-depth } \\
\text { function }
\end{array}\right)\left(\begin{array}{c}
\text { rms magnetic-field- } \\
\text { strength factor }
\end{array}\right)\left(\begin{array}{c}
\text { density } \\
\text { factor }
\end{array}\right) \mathrm{R}^{\mathrm{n}} \\
& \text { Power }=\left(\begin{array}{c}
\text { coil-geometry } \\
\text { function }
\end{array}\right)\left(\begin{array}{c}
\text { skin-depth } \\
\text { function }
\end{array}\right)\left(\begin{array}{c}
\text { rms magnetic-field- } \\
\text { strength factor }
\end{array}\right)\left(\begin{array}{c}
\text { conductivity } \\
\text { factor }
\end{array}\right) \mathrm{R}^{\mathrm{m}_{;}}
\end{aligned}
$$




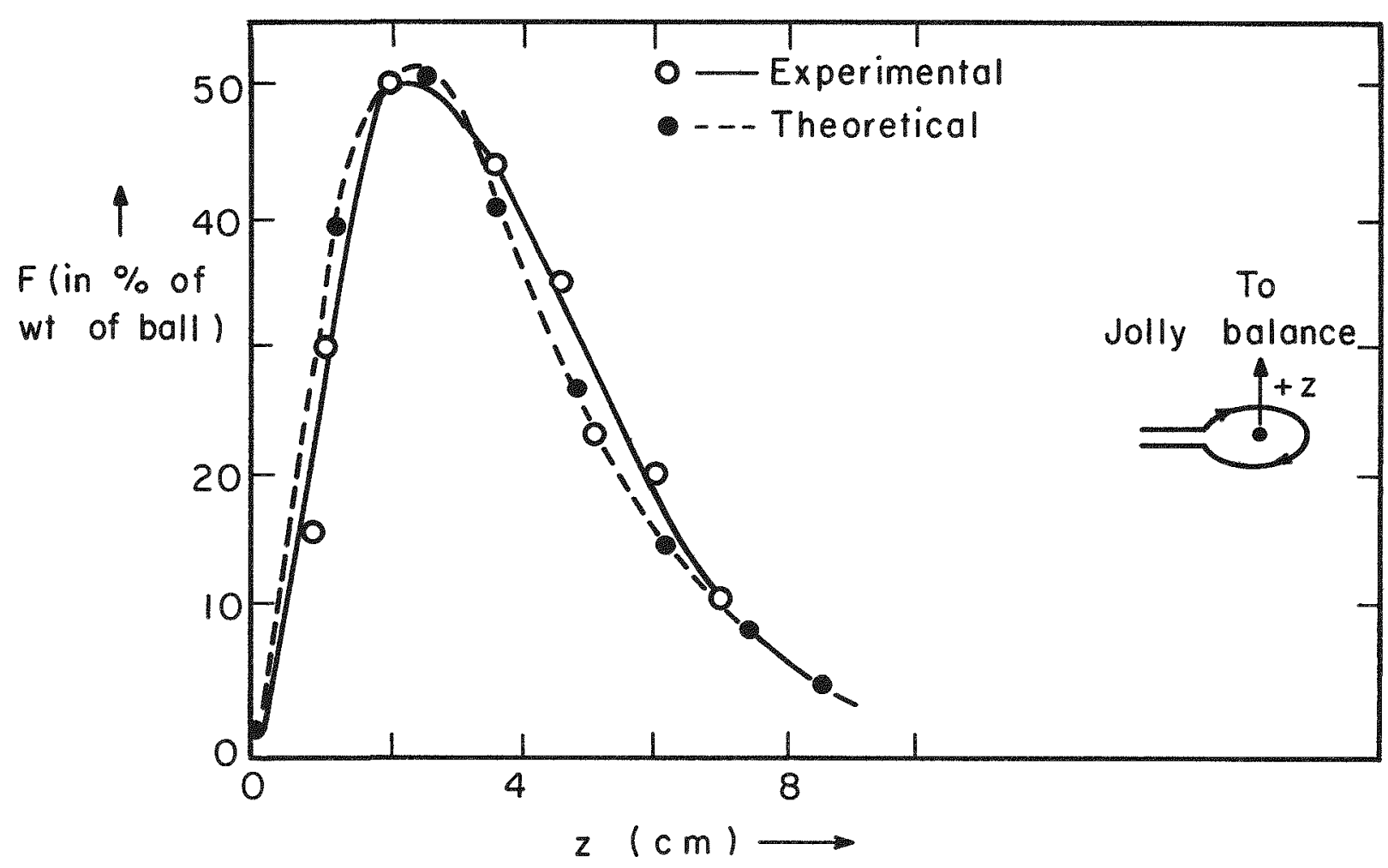

Lifting force vs position

Fig. 5. Lifting force on a solid metal sphere exerted by alternating magnetic field of a single circular loop along its axis. 


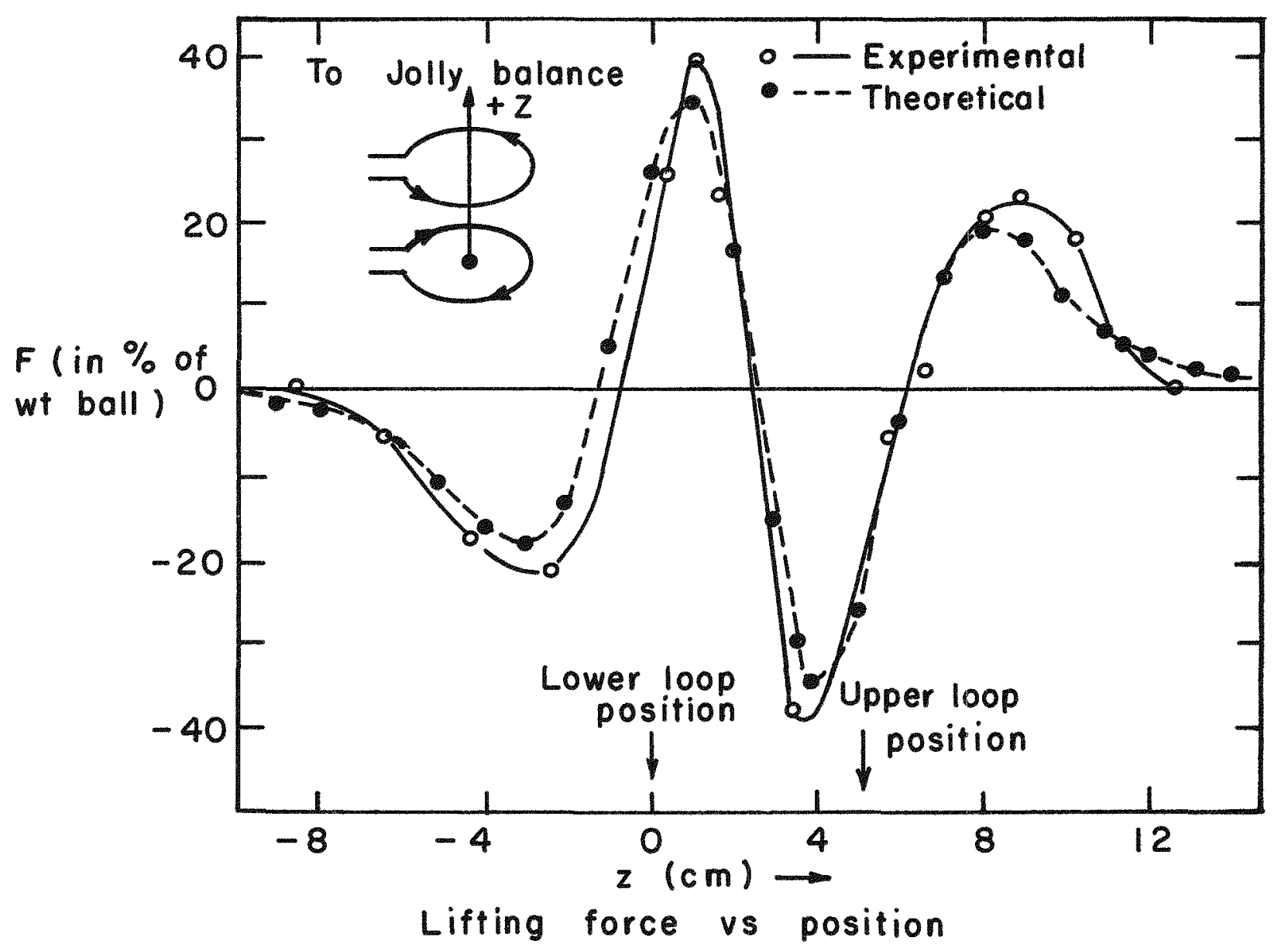

MUB - 3019

Fig. 6. Lifting force on a solid metal sphere exerted by alternating magnetic field of two coaxial circular loops (with current directions opposed as shown) along their common axis. 


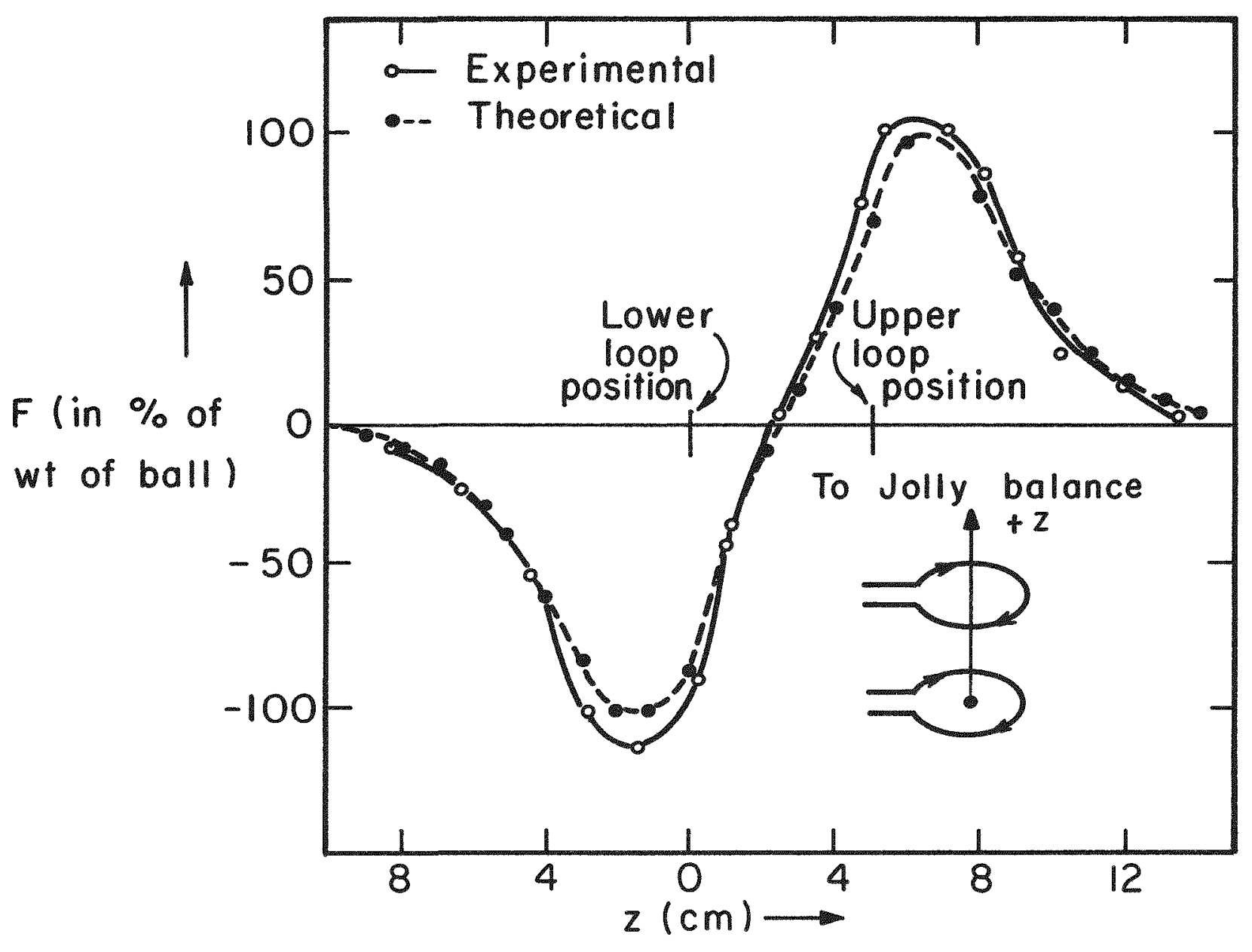

Lifting force vs position

MUB -3020

Fig. 7. Lifting force on a solid metal sphere exerted by alternating magnetic field of two coaxial circular loops (with current directions the same as shown) along their common axis. 


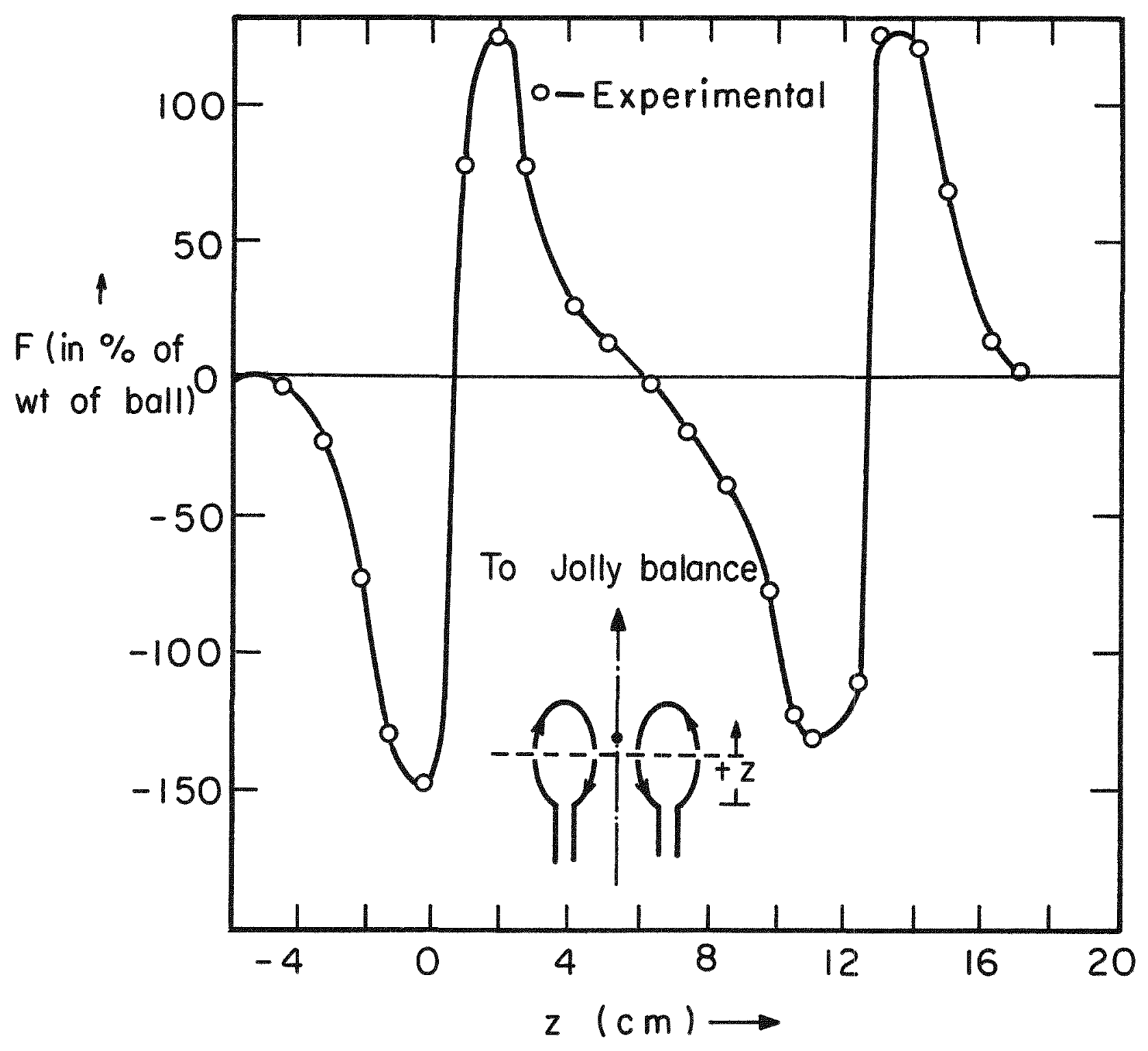

Lifting force vs position

Fig. 8. Lifting force on a solid metal sphere exerted by alternating magnetic field of two coaxial circular loops (with current directions opposed as shown) along a loop radius projected onto plane midway between planes of the loops. 
where $n$ and $m$ are specified integral powers of the sphere radius $R$. The significance of this type of formulation is that both the force and power equations can be divided into two independent and separable multiplicative functions, one relating to the geometry of the field distribution and the other relating to the value of the skin depth. These functions are different in the force and power equations, a result that has important consequences for the practical application of levitation.

\section{Definition of $\mathrm{G}(\mathrm{x}), \mathrm{H}(\mathrm{x})$, and $\mathrm{F}(\mathrm{x})$}

The skin-depth functions, $G(x)$ and $H(x)$, can be immediately discussed without reference to any particular field distribution or intensity. These are the two basic functions of levitation theory. For purposes of discussion, it is convenient to define their ratio,

$$
F(x)=\frac{G(x)}{H(x)}
$$

$F(x)$ is a measure of the effect of the skin depth on the force-to-power ratio for the sphere.

In Figs. 9 through 13, the quantities $G(x), H(x)$, and $F(x)$ are plotted for both low and high values of $\mathrm{x}$. The correct formulas for these functions for nonferrous metals are

$$
\begin{aligned}
& G(x)=1-\frac{3}{2 x} \frac{\sinh 2 x-\sin 2 x}{\cosh 2 x-\cos 2 x} \\
& H(x)=\frac{x(\sinh 2 x+\sin 2 x)}{\cosh 2 x-\cos 2 x}-1 \\
& F(x)=\frac{2 x(\cosh 2 x-\cos 2 x)-3(\sinh 2 x-\sin 2 x)}{2 x^{2}(\sinh 2 x+\sin 2 x)-2 x(\cosh 2 x-\cos 2 x)}
\end{aligned}
$$




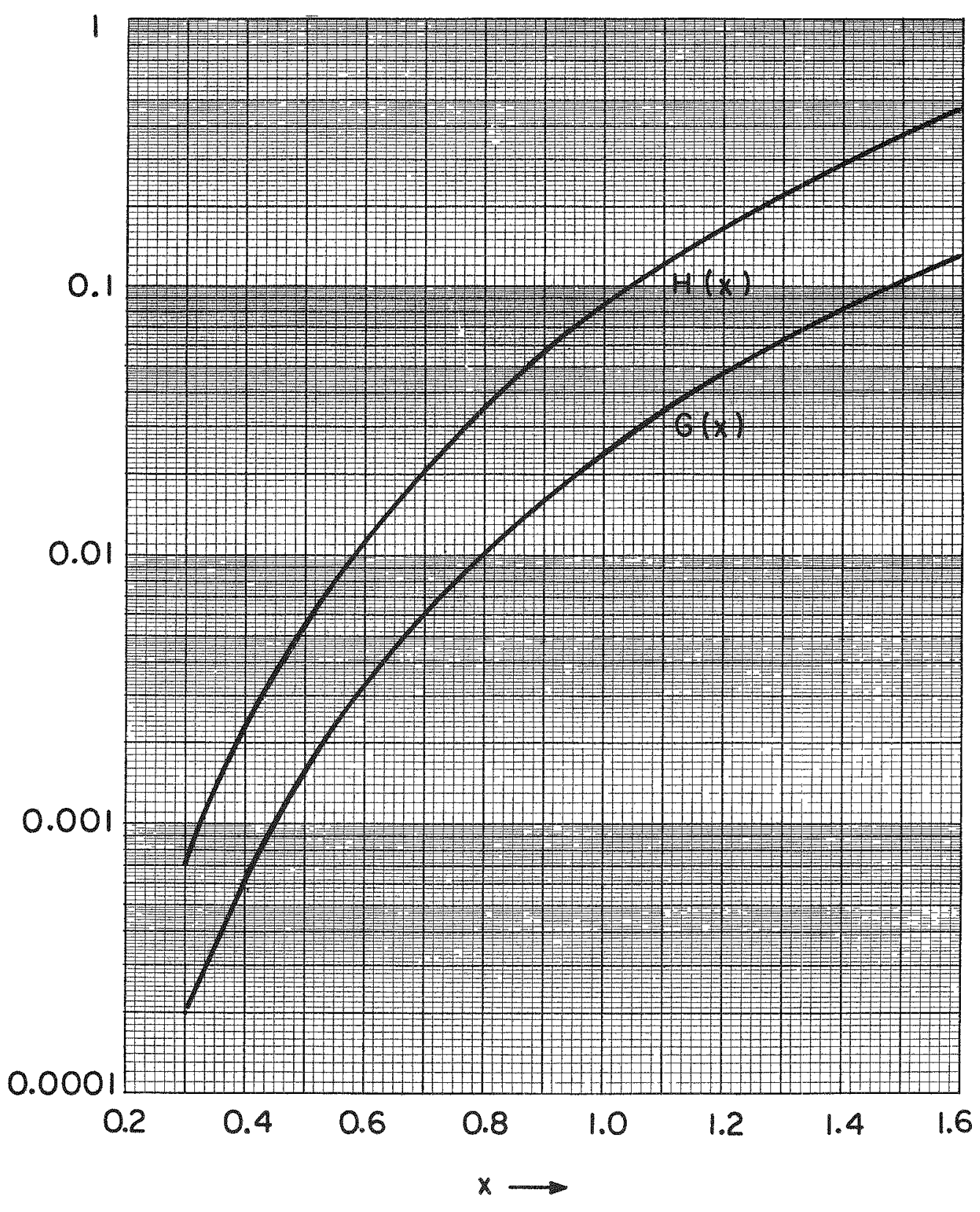

$M \cup B-3034$

Fig. 9. $G(x)$ and $H(x)$ plotted as a function of $x$ (low values of $x$ ). 


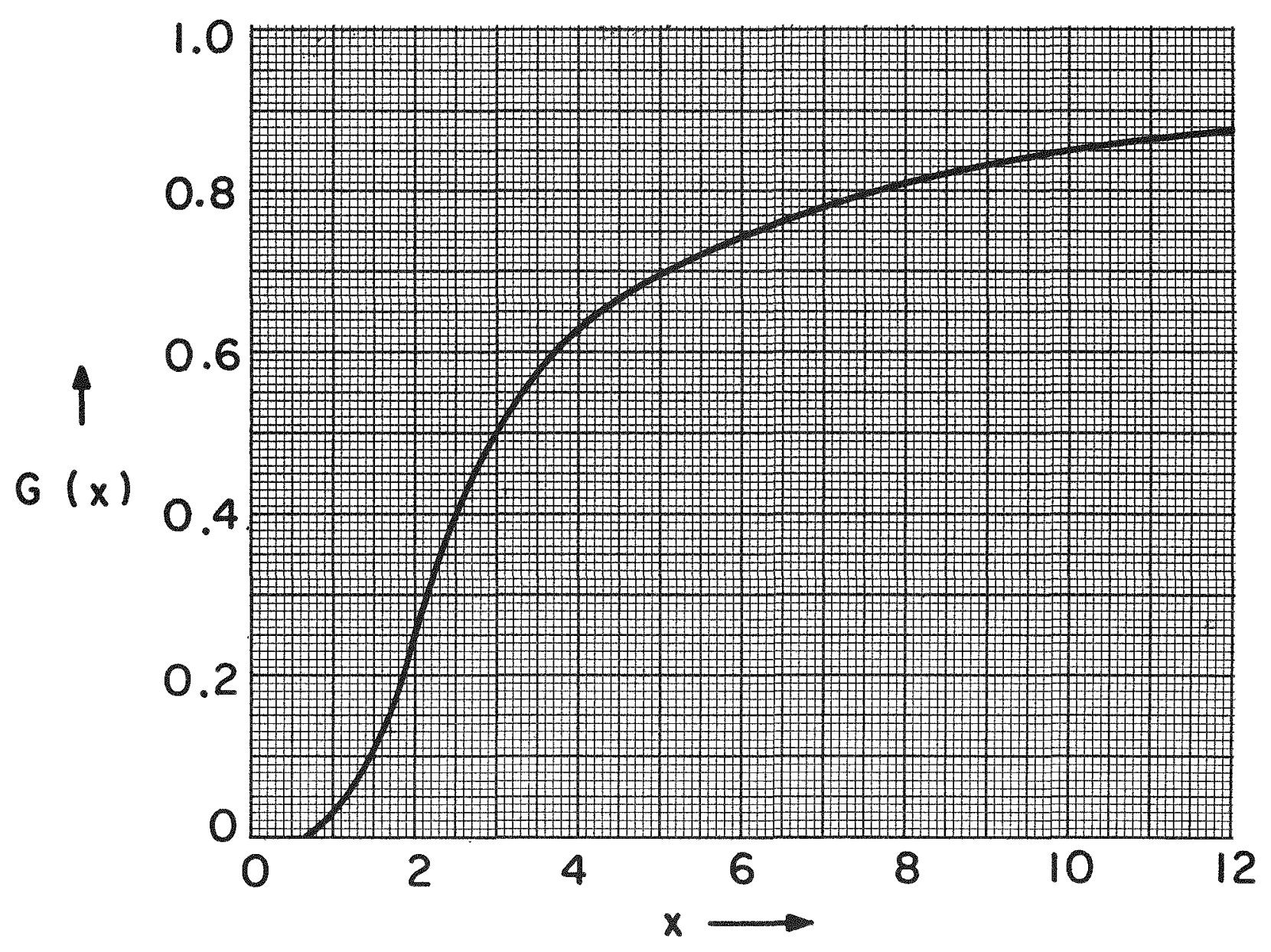

MUB -3035

Fig. 10. $G(x)$ plotted as a function of $x$ (high values of $x$ ), where $G(x)=1-\frac{3}{2 x} \frac{\sinh 2 x-\sin 2 x}{\cosh 2 x-\cos 2 x}$. 


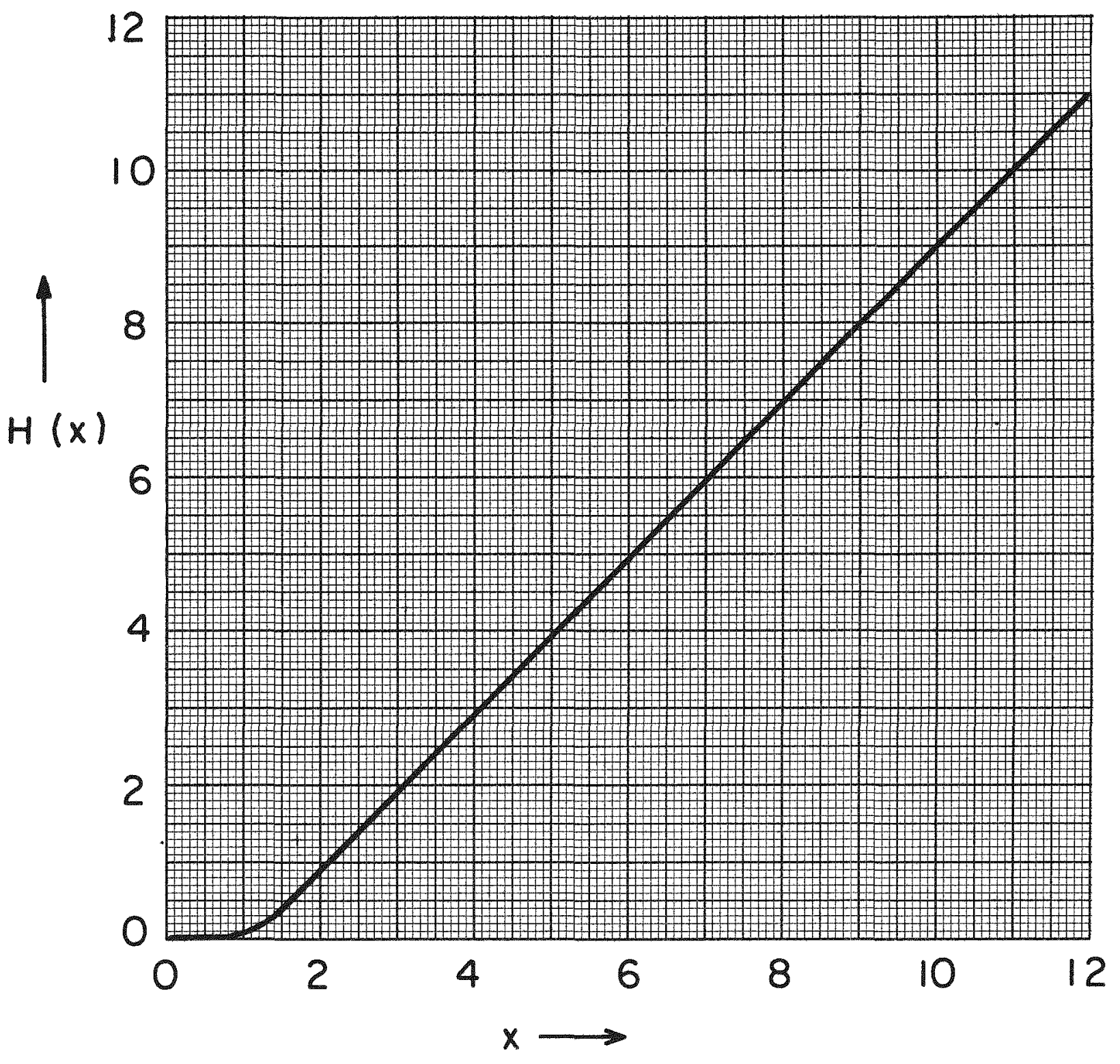

MUB -3036

Fig. 11. $H(x)$ plotted as a function of $x$ (high values of $x$ ), where

$$
H(x)=\frac{x(\sinh 2 x+\sin 2 x)}{\cosh 2 x-\cos 2 x}-1
$$




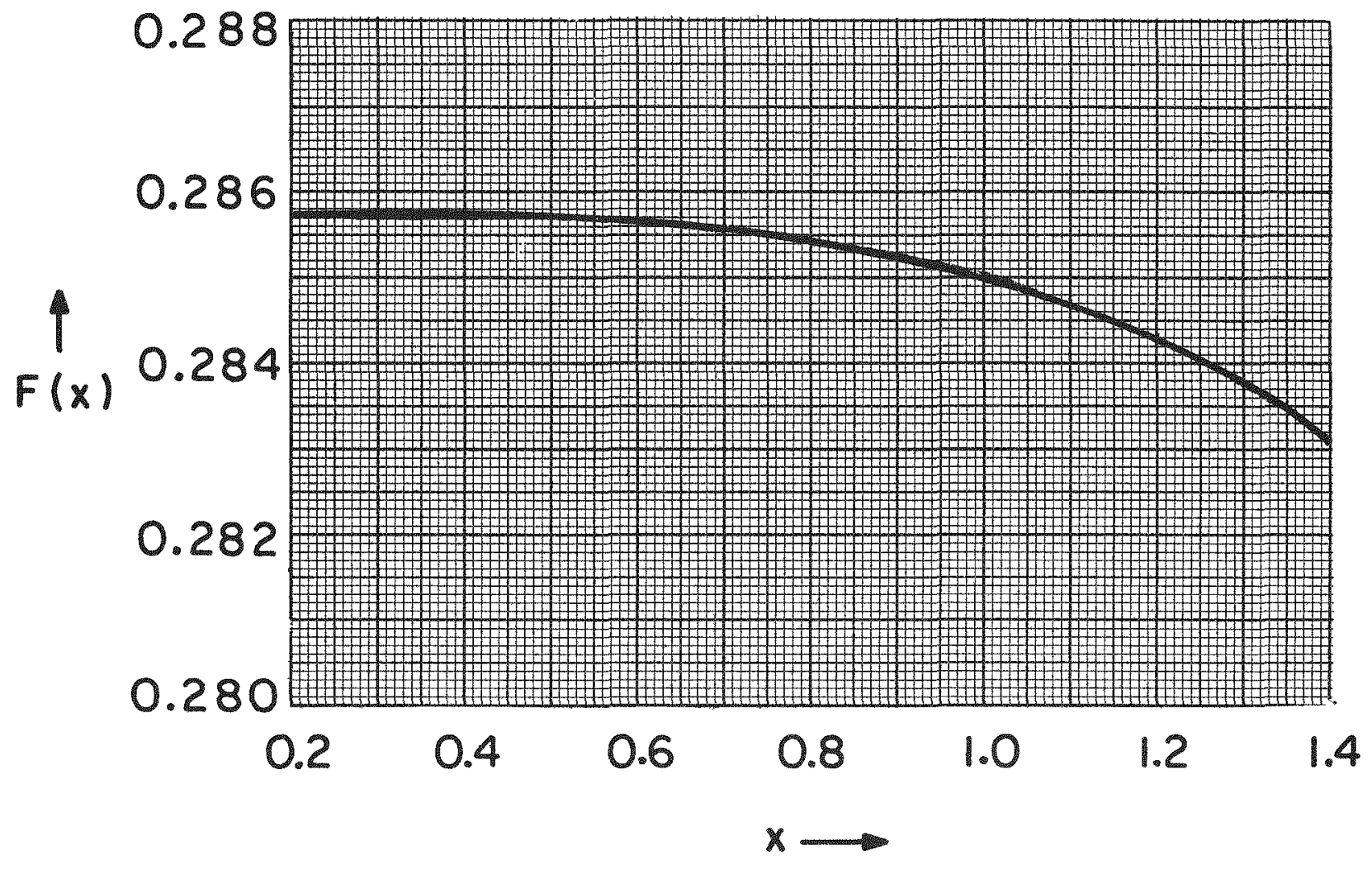

$M U B-3037$

Fig. 12. $F(x)$ plotted as a function of $x$ (low values of $x$ ). 


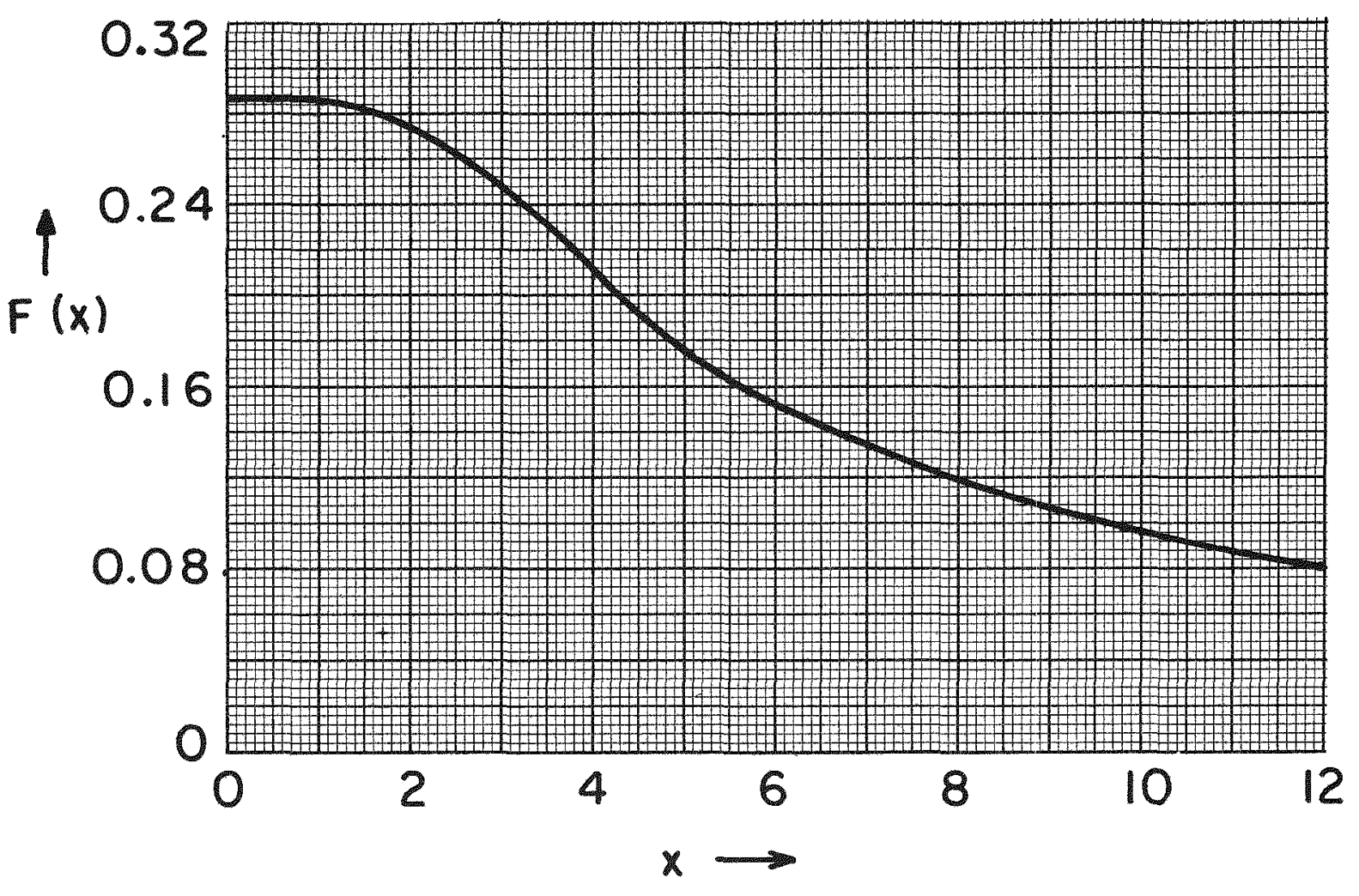

MUB -3038

Fig. 13. $F(x)$ plotted as a function of $x$ (high values of $x$ ), where

$$
F(x)=\frac{G(x)}{H(x)} \text {. }
$$


In the limit of large $x, x \geqslant 2$, they reduce to

$$
\begin{aligned}
& G(x) \approx 1-\frac{3}{2 x} \\
& H(x) \approx x-1 \\
& F(x) \approx \frac{2 x-3}{2 x(x-1)} .
\end{aligned}
$$

For low values of $x$, they are given by the approximate formulas

$$
\begin{aligned}
& G(x) \approx 0.025 x^{4} \\
& H(x) \approx 0.091 x^{4} \\
& F(x) \approx 0.28,
\end{aligned}
$$

where $\mathrm{x} \leqq 1$

Equations (1) and (5) show that the parameter $\mathrm{x}$ is proportional to the square root of the frequency. Thus, the higher the frequency, the larger the value of $x$. In the limit as $x$ becomes very large, the force function $G(x)$ approaches 1.000 at relatively low values of $x$, whereas the heating function $H(x)$ increases essentially without bound. For metals of moderate electrical conductivity, no significant increase in the levitation force results by operation at very high frequencies. At these very high frequencies, however, the energy a.bscrbed by the sphere becomes so large that the sample melts and may even Bburn up. Because of this, the use of high frequencies is ideal for inductionheating applications. Figures 12 and 13 show that the ratio of the force and heating functions, $F(x)$, is relatively constant for low values of $x$. From these observations, the following two conclusions can be made:

a. All other conditions of field geometry and magnitude being the same, if it is desired to levitate a metal but not to heat it, "low" frequencies and high field strengths and gradients should be used. 
b. Alternatively, if only heating is desired, moderate field strengths and high to very high frequencies should be used.

These conclusions define quite explicitly the useful operating regions for levitation and induction heating, respectively. The importance of frequency in such applications is certainly apparent from the above considerations. The exact value of the "low" frequency to be used depends completely on the nature of the metal, the magnitude of the radius, and the operating temperature desired (see Charts 6 and 12). For a $1-\mathrm{cm}$ radius sphere of liquid mercury at $20^{\circ} \mathrm{C}$, the frequency should be about $40 \mathrm{kc} / \mathrm{sec}$.

\section{Effect of Coil Geometry}

To demonstrate how the coil geometry affects the levitation experiment, consider a metal sphere levitated in a field produced by two coaxial loops, separated by a distance $2 \mathrm{~d}$, in which opposed alternating currents are present (Fig. 14). From simple magnetostatic considerations such as those given by Smythe ${ }^{17}$ or Van Blade1, ${ }^{18}$ the $r$ and $z$ components of the field are

$$
\begin{gathered}
B_{z}=B_{z I}+B_{z I I}=\frac{\mu_{0}}{2 R_{1}}\left[\frac{I_{I}}{\left(1+y_{I}\right)^{3 / 2}}+\frac{I_{I I}}{\left(1+y_{I I}^{2}\right)^{3 / 2}}\right] \\
B_{r}=B_{r I}+B_{r I I}=\frac{\mu_{0}}{2 \pi R_{1}}\left\{\frac{y_{I}^{I}}{\rho\left[(1+\rho)^{2}+y_{I}^{2}\right]^{1 / 2}}\left[-K_{I}+\frac{1+\rho y_{I}^{2}}{(1-\rho)^{2}+y_{I}^{2}} E_{I}\right]\right. \\
\left.+\frac{y_{I I I I}^{I}}{\rho\left[(1+\rho)^{2}+y_{I I}^{2}\right]^{1 / 2}}\left[-K_{I I}+\frac{1+\rho^{2}+y_{I I}^{2}}{(1-\rho)^{2}+y_{I I}^{2}} E_{I I}\right]\right\}
\end{gathered}
$$

where $\mathrm{K}$ and $\mathrm{E}$ are complete elliptic integrals of the first and second kinds of the moduli 


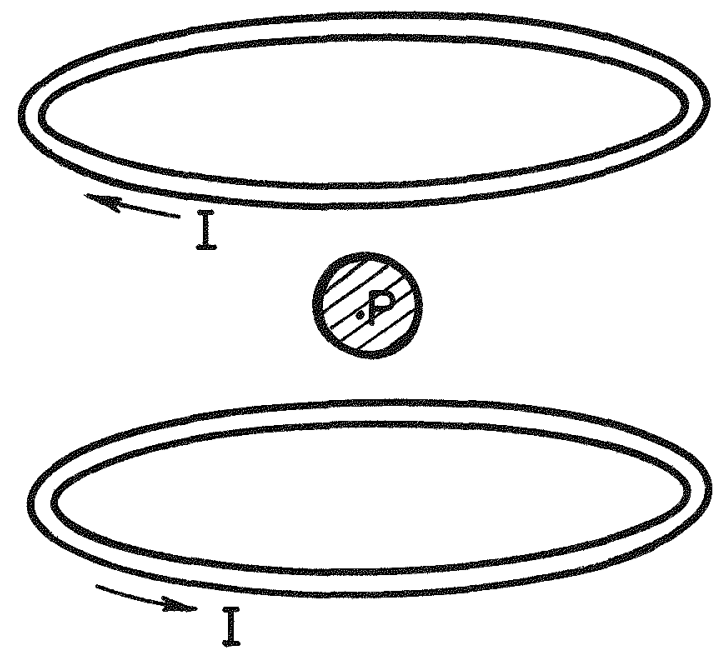

MUB -3022

Fig. 14. Metal sphere levitated at point $P$ between two coaxial loops with opposed current directions. 


$$
k_{I}^{2}=\frac{4 \rho}{(1+\rho)^{2}+y_{I}^{2}} \quad \text { and } \quad k_{I I}^{2}=\frac{4 p}{(1+\rho)^{2}+y_{I I}^{2}}
$$

$I_{I}$ and $I_{I I}$ are the currents in coils $I$ and II, respectively, and $\rho, y_{I}$, and $y_{I I}$ are'defined as

$$
y_{I}=\frac{z+d}{R_{1}} \quad y_{I I}=\frac{z-d}{R_{1}} \quad \rho=\frac{r}{R_{1}} .
$$

When $z=0$, the following simplifications appear:

$$
\begin{aligned}
& \mathrm{y}_{I}=-\mathrm{y}_{\mathrm{II}}=\mathrm{y}=\frac{\mathrm{d}}{\mathrm{R}_{1}} \\
& \mathrm{k}_{\mathrm{I}}^{2}=\mathrm{k}_{\mathrm{II}}^{2}=\mathrm{k}^{2} \\
& \mathrm{~K}_{\mathrm{I}}=\mathrm{K}_{\mathrm{II}}=\mathrm{K} \\
& \mathrm{E}_{\mathrm{I}}=\mathrm{E}_{\mathrm{II}}=\mathrm{E} .
\end{aligned}
$$

Also, if the alternating currents in coils I and II are opposed to each other,

$$
I_{I}=-I_{I I}=I \text {. }
$$

With these simplifications, the expressions for the field and field gradients in the $z$ and $r$ directions become

$$
\begin{gathered}
B_{z}=B_{z I}-B_{z I I}=0 \\
\frac{\partial B_{z}}{\partial z}=2 \frac{\partial B_{z I}}{\partial z}=2 \frac{\partial B_{z I I}}{\partial z}=-\frac{3 \mu_{0} I}{R_{1}^{2}} \frac{y}{\left(1+y^{2}\right)^{5}} \\
B_{r}=2 B_{r I}=2 B_{r I I}=\frac{\mu_{0} I}{\pi R_{1}^{2}} \frac{y}{p\left[(1+p)^{2}+y^{2}\right]^{1 / 2}}\left[-K+\frac{1+p^{2}+y^{2}}{(1-p)^{2}+y^{2}}\right]
\end{gathered}
$$




$$
\frac{\partial B_{r}}{\partial r}=2 \frac{\partial B_{r I}}{\partial r}=2 \frac{\partial B_{r I I}}{\partial r} .
$$

Therefore, in the $\mathrm{z}$ direction the gradients are additive and the fields tend to cancel each other. In the $r$ direction, both the fields and the field gradients are additive. The origin is taken at a point on the axis midway between the two coils, so tile above considerations result directly from the symmetry of the system.

According to Eqs. (3) and (4), the levitation force on a metal sphere is proportional to the product of the field and field gradient, and the power is proportional to the square of the field intensity. Thus, there is a greater force and a greater rate of heating acting on the metal sphere when the axis of the two colls is horizontal instead of vertical. The first of these conclusions nas been verified experimentally by okress et al. ${ }^{19}$ A comparison of their data, shown in Figs, 6 and 8 , demonstrates that the maximum force on the I-in. bronze ball is approximately 3.5 times greater when the axis of the twocoil system is horizontal than when it is vertical.

In practice, the coil turns have a finite thickness and the above formulas do not strictly apply. Fortunately, a very thorough analysis of this particular point as well as the geometrical design and characteristics of commonly used levitation coils has been given by Hulsey. 20 


\section{APPLICATION OF THE LEVITATION EQUATIONS}

\section{A. Simplified Levitation Equations}

Equations (3) and (4) can be simplified along the lines of Eqs. (6) and (7),

$$
\begin{aligned}
& \frac{F}{W}=\lambda_{1} B^{2} \frac{G(x)}{\rho} \\
& P=\lambda_{2} B^{2} \frac{H(x)}{\sigma},
\end{aligned}
$$

where $\lambda_{1}$ and $\lambda_{2}$ depend on the spatial characteristics of the magnetic field, the size of the levitated sphere, and the point in the field at which it is levitated, and $B^{2}$ is defined by

$$
B^{2}=\frac{\mu_{0}^{2} I^{2}}{4 R_{1}^{2}}
$$

For the coil system shown in Fig. 13 and considered in Sec. II. D., the values of the parameters $\lambda_{1}$ and $\lambda_{2}$ are

$$
\begin{gathered}
\lambda_{1}=\frac{9}{2 R_{1} \mu_{0}}\left[\frac{1}{\left(1+y_{I}^{2}\right)^{3 / 2}}-\frac{1}{\left(1+y_{I I}^{2}\right)^{3 / 2}}\right]\left[\frac{y_{I}}{\left(1+y_{I}^{2} 5 / 2\right.}-\frac{y_{I I}}{\left(1+y_{I I}^{2}\right)^{5 / 2}}\right] \\
\lambda_{2}=\frac{3 \pi R_{2}}{\mu_{0}^{2}}\left[\frac{1}{\left(1+y_{I}^{2}\right)^{3 / 2}}-\frac{1}{\left(1+y_{I I}^{2}\right)^{3 / 2}}\right]^{2}
\end{gathered}
$$

For a particular metal sphere levitated at constant temperature at various points in a given magnetic-field, only the values of $\lambda_{1}, \lambda_{2}$, and $B$ are needed to describe the relative behavior at the different points. For spheres of identical size but different metals located in the same magnetic field and levitated at the same point, only the values of $p, \sigma, x$, and $B$ are needed to describe the relative behavior of the different metals. These two cases are considered in Secs. III. B and III. C. 


\section{B. Typical Levitation Experiment}

The current in the coils producing a given magnetic-field distribution and intensity usually is controlled by one knob, the current-control knob on the induction heater. With this one knob, attempts are made to control both the force on and the heating of a metal sphere levitated by the field. The frequent failure to achieve this control has been one of the chronic deficiencies of levitation heating. It is therefore worthwhile to discuss the pitfalls inherent in such an attempt.

Consider the case of a metal sphere levitated in a vacuum at point a by a given magnetic-field distribution and intensity, as illustrated in Figs 。 15 and 16. Since the sphere is exactly levitated,

$$
\text { Force }=\text { Weight. }
$$

From Eqs. (25) and (26), the force and power are

$$
\begin{aligned}
& \lambda_{1 a} B_{a}^{2} \frac{G(x)}{\rho}=1 \\
& P_{a}=\lambda_{2 a} B_{a}^{2} \frac{H(x)}{\sigma} .
\end{aligned}
$$

What happens when the induction-heater current is (a) increased, (b) decreased, and (c) left unchanged?

When the current in the loops is increased, the metal sphere is lifted to a new point $b$ in the field. Equations (25) and (26) are now

$$
\begin{aligned}
& \lambda_{1 b} B_{b}^{2} \frac{G(x)}{\rho}=1 \\
& P_{b}=\lambda_{2 b} B_{b}^{2} \frac{H(x)}{\sigma},
\end{aligned}
$$

where $B_{b}>B_{a}$ and $\lambda_{1 b}<\lambda_{1 a}$. With the field distributions used in typical levitation applications, it is usually observed that

$$
\lambda_{2 b} B_{b}^{2}<\lambda_{2 a} B_{a}^{2}
$$




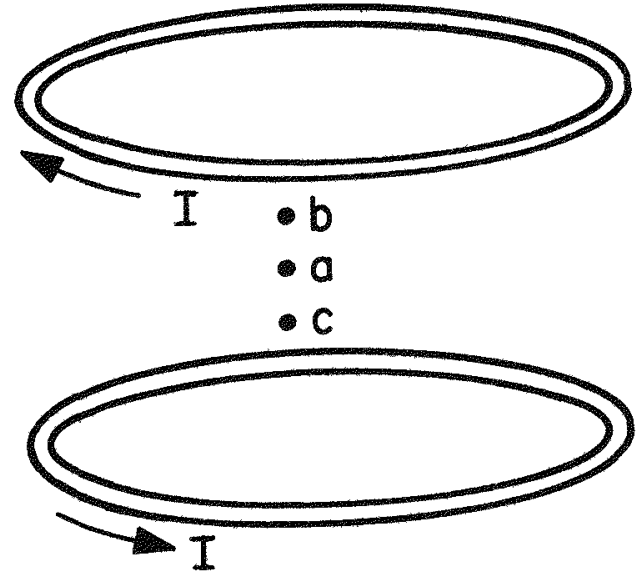

$M \cup B-3023$

Fig. 15. Location of points $a, b$, and $c$ in the coaxial-1oop levitation system. 


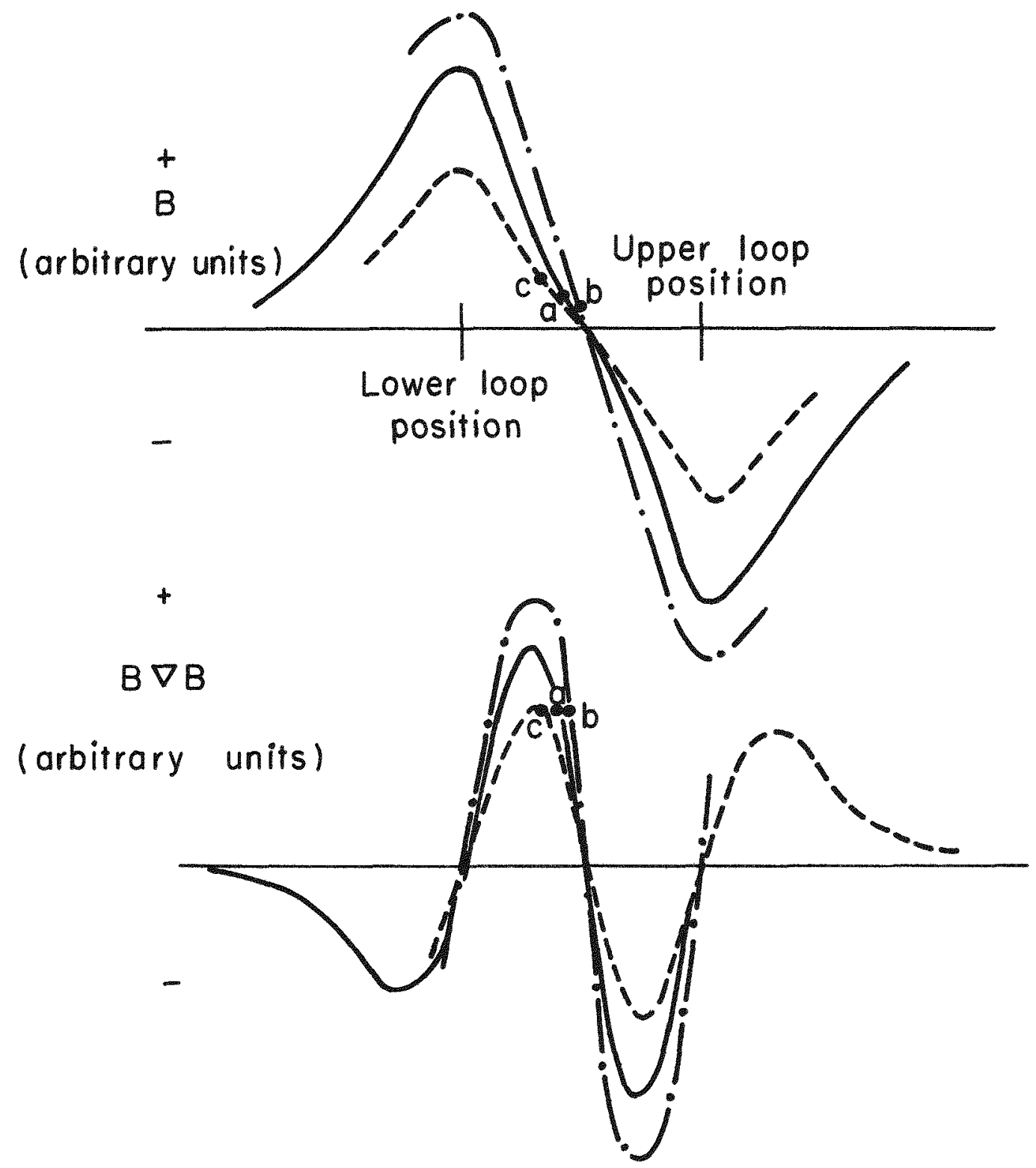

MUB -3024

Fig. 16. Schematic plots of $B$ and $B \nabla B$ along the common axis for two coaxial circular loops with opposed current directions.

- initial coil current, _- - decreased coil current, - - increased coil current. 
with the result that the power actually decreases

$$
\mathrm{P}_{\mathrm{b}}<\mathrm{P}_{\mathrm{a}}
$$

causing the metal to decrease in temperature despite the increase in coil current. This phenomenon is illustrated in graphical form in Fig. 15.

When the current is decreased, the force on the metal is diminished and it drops to a lower point $c$ in the field. Now the relationships are

$$
\begin{aligned}
& \lambda_{1 c} B_{c}^{2} \frac{G(x)}{\rho}=1 \\
& P_{c}=\lambda_{2 c} B_{c}^{2} \frac{H(x)}{\sigma} \\
& B_{c}<B_{a} \\
& \lambda_{1 c}>\lambda_{1 a} \\
& \lambda_{2 c} B_{c}^{2}>\lambda_{2 a} B_{a}^{2} \\
& P_{c}>P_{a} .
\end{aligned}
$$

Thus, despite the decrease in coil current, the temperature of the metal increases.

Finally, if the current is left unchanged and if the metal is levitated in a vacuum environment, it gradually increases in temperature due to the energy being absorbed. As it increases in temperature, the metal resistivity also increases, with the result that $G(x)$, and consequently the force, decreases. The metal drops to lower positions in the coil field distribution where the power absorption is greater. The value of $x$, and consequently the force, continues to decrease until either thermal steady state is reached or else the metal falls 
or pours out of the magnetic-field region. In vacuums, the latter usually occurs. In the presence of gases of high thermal conductivity (such as helium and hydrogen), thermal steady state can be achieved and the metal can be maintained at any reasonable temperature in the solid or liquid states. 11,21

The conclusion that can be derived from the above description of a. typical levitation experiment is

FOR LONG DURATIONS, THE FORCE ON AND THE TEMPERATURE OF A METAL LEVITATED IN A VACUUM CANNOT BE SIMULTANEOUSLY CONTROLLED WITH ONLY THE CURRENT -CONTROL KNOB ON THE INDUCTION HEATER.

The restriction "long duration" in this conclusion must be emphasized. Because of the fortuitous design of a levitation coil, the use of small samples, the choice of metal, or the use of low frequencies, it is possible to levitate certain solid and liquid metals as long as 20 or $30 \mathrm{~min}$ in a vacuum. The procedures for achieving such stability have not been completely elucidated, so this area of levitation can still be considered an art.

For achieving the long-duration levitation of metals at a specifically chosen temperature, the only alternatives are

a. Levitate at high frequencies, but have a separate method of cooling the metal sphere and establishing thermal steady-state conditions. An example is the use of gases of high thermal conductivity.

3. Levitate at "low" frequencies, but have a separate method of heating the metal sphere and establishing thermal steady-state conditions. An example is the use of an arc, electron beam, or small high-frequency induction-heating coil.

In both of these alternatives, independent controls on the levitation force and the metal temperature are achieved. This is the basic requirement for a satisfactory levitation system. For levitation experiments in a vacuum, 
only the second alternative is available. It will be shown in Sec. III. C that individual metals vary in their energy-absorption properties. Thus, the applicability and usefulness of the above alternatives depend entirely on the metal levitated.

For applications such as alloy preparation, sintering, vapor plating, and metal purification, the necessity for long duration levitation of the metal sample is not so great. This fact is the basis for the usefulness of the existing commercial levitation units.

\section{Levitation of Different Metals}

Along with the use of only one knob to control both the force on and the heating of a metal sample, only one fixed frequency, $450 \mathrm{kc} / \mathrm{sec}$, is used in commercial levitation devices to hopefully levitate different sizes and shapes of some 60 different metals and countless more alloys. The varying levitation behavior of the different metals tried and the inability to compensate for this variation has been another one of the major deficiencies of the levitation heating technique. In addition, although considerable attention has been given to the design and construction of levitation coils, no systematic attempt has been made to calculate the relative ease of levitation of different metals. It is thus worthwhile to inquire into the limitations on the types of metals or semiconductors that can be levitated and the usefulness of operating at frequencies other than $450 \mathrm{kc} / \mathrm{sec}$.

Consider the case of spheres of different metals with identical radii $R$ levitated at the same point in the same magnetic-field distribution in separate experiments. The metals differ from each other only in the values of their density and electrical conductivity. Since each sphere is exactly levitated, formula (30)

$$
\text { Force }=\text { Weight }
$$


applies, as do Eqs. (25) and (26)

$$
\begin{aligned}
& \lambda_{1} B^{2} \frac{G(x)}{\rho}=1 \\
& P=\lambda_{2} B^{2} \frac{H(x)}{\sigma} .
\end{aligned}
$$

Two distinct cases can now be considered:

1. Operation at a constant skin depth for every metal, and

2. Operation at constant frequency for every metal.

Case 1

In this case, $\lambda_{1}, \lambda_{2}, G(x)$, and $H(x)$ are constant for every metal. A suitable value for the parameter $x$ is 4.0 , which corresponds to $G(x)=0.625$ and $H(x)=3.00$. Solid sodium, one of the easiest metals to levitate, is chosen as a standard. The field needed to levitate it is given by the formula

$$
\mathrm{B}_{\mathrm{Na}}^{2}=\frac{\rho_{\mathrm{Na}}}{\lambda_{1} \mathrm{G}(\mathrm{x})} .
$$

The field needed to levitate any other metal, Me, is given by

$$
\mathrm{B}_{\mathrm{Me}}^{2}=\frac{\rho_{\mathrm{Me}}}{\rho_{\mathrm{Na}}} \mathrm{B}_{\mathrm{Na}}^{2} \text {. }
$$

The power for the different metal spheres is

$$
\begin{aligned}
& P_{\mathrm{Na}}=\frac{\lambda_{2}}{\lambda_{1}} \frac{\rho_{\mathrm{Na}}{ }^{\top} \mathrm{Na}}{\mathrm{F}(\mathrm{x})}
\end{aligned}
$$

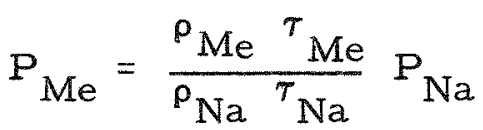

where $\tau=1 / \sigma$. 
Case 2

If the metals are levitated at constant frequency, the skin-depth functions $G(x)$ and $H(x)$ no longer remain the same for each metal. Now, the magnetic field needed to levitate the spheres is

$$
\begin{aligned}
\mathrm{B}_{\mathrm{Na}}^{2} & =\frac{\rho_{\mathrm{Na}}}{\lambda_{1} \mathrm{G(x_{ \textrm {Na } } )}} \\
\mathrm{B}_{\mathrm{Me}}^{2} & =\frac{\rho_{\mathrm{Me}}{ }^{\mathrm{G}\left(\mathrm{x}_{\mathrm{Na}}\right)}}{{ }_{\mathrm{Na}}^{\mathrm{G}\left(\mathrm{x}_{\mathrm{Me}}\right)}} \mathrm{B}_{\mathrm{Na}}^{2},
\end{aligned}
$$

and the power is

$$
\begin{aligned}
& P_{\mathrm{Na}}=\frac{\lambda_{2}}{\lambda_{1}} \frac{p_{\mathrm{Na}}{ }^{\tau} \mathrm{Na}}{\mathrm{F}\left(\mathrm{x}_{\mathrm{Na}}{ }^{l}\right.} \\
& P_{\mathrm{Me}}=\frac{\rho_{\mathrm{Me}} \tau_{\mathrm{Me}} F\left(\mathrm{x}_{\mathrm{Na}}\right)}{\rho_{\mathrm{Na}}{ }^{\tau} \mathrm{Na}{ }^{\mathrm{F}\left(\mathrm{x}_{\mathrm{Me}}\right)}} \mathrm{P}_{\mathrm{Na}} .
\end{aligned}
$$

At low values of $x$, the ratio $\left[F\left(x_{N a}\right] /\left[F\left(x_{M e}\right)\right]\right.$ may not vary much for different metals. For the levitation of 1 -cm-radius spheres of silver, sodium, and graphite at $10 \mathrm{kc} / \mathrm{sec}$,

$$
\frac{F\left(x_{N a}\right)}{F\left(x_{A g}\right)}=1.66 \text { and } \frac{F\left(x_{N a}\right)}{F\left(x_{C}\right)}=0.344 .
$$

All other metals fall between these two limits at $10 \mathrm{kc} / \mathrm{sec}$. Thus, the power for the metals in both cases can be approximated by Eq. (44).

The fields needed to levitate the metals differ widely for the two cases. The ratio $\left[G\left(x_{N a}\right)\right] /\left[G\left(x_{M e}\right)\right]$ for $1-\mathrm{cm}$ spheres of silver, sodium, and graphite at $10 \mathrm{kc} / \mathrm{sec}$ is

$$
\frac{G\left(x_{N a}\right)}{G\left(x_{A g}\right)}=0.932 \text { and } \frac{G\left(x_{N a}\right)}{G\left(x_{C}\right)^{\prime}}=137 \text {. }
$$


From Eq. (46), it is seen that at $10 \mathrm{kc} / \mathrm{sec}$ exceedingly high fields are needed to levitate a 1 -cm-radius sphere of graphite. At $450 \mathrm{kc} / \mathrm{sec}$, these ratios are

$$
\frac{G\left(x_{N a}\right)}{G\left(x_{A g}\right)}=0.99 \text { and } \frac{G\left(x_{N a}\right)}{G\left(x_{C}\right)}=1.43 .
$$

When levitating graphite, there is a definite advantage to operating at this frequency instead of $10 \mathrm{kc} / \mathrm{sec}$ : The magnetic field strength required at 450 $\mathrm{kc} / \mathrm{sec}$ is less by a factor of ten.

By calculations such as these, it is possible to determine an optimum frequency, or, more correctly, a minimum frequency for the levitation of a metal of specified size and electrical conductivity. Operation at frequencies considerably greater than this frequency has the following disadvantages:

1. Coil power losses are increased.

2. It is more difficult to obtain high-magnetic-field strengths.

3. The sample may become excessively heated and difficult to control.

4. Ferrites and high-permeability laminated-core materials are less us eful.

5. It is more difficult to employ repetitively switched solid-state power devices.

Operation at frequencies considerably lower than this frequency has the following fundamental disadvantage: Considerably greater magnetic field strengths must be used.

This is the explanation for the distinction "near-optimum frequency" given to the frequencies listed for 1 - cm-radius metal spheres in Charts 6 and 12. Considerations of cost, feasibility, efficiency, and sample control should not substantially alter this choice of frequency. A change of size of the metal sphere, by virtue of Eq. (5), definitely alters it. 
To demonstrate the practical applications of Eqs. (42), (44), (46), and (48), it is convenient to summarize the data and calculations for more than 50 different elements by a series of periodic charts. Chart 1 gives the density of the elements at $20^{\circ} \mathrm{C},{ }^{22}$ and Chart 2 gives their electrical resistivities at $0^{\circ} \mathrm{C} .{ }^{23}$ The values of the element densities can be used directly in Eq. (42) to calculate the field ratios.

Chart 3 lists the product of these two quantities, a result that can be used to compute the power ratios given in Eqs. (44) and (48). For the purpose of these levitation calculations, the difference in the density of the metals between $0^{\circ} \mathrm{C}$ and $20^{\circ} \mathrm{C}$ is insignificant.

Chart 4 gives the values of the function, $G(x) / \rho$, for $1-c m$-radius metal spheres in a $10 \mathrm{kc} / \mathrm{sec}$ magnetic field. This quantity can be used in Eq. (46) to determine the field ratios required to levitate the metals, in comparison with sodium, at the frequency given. For comparison, Chart 5 gives the values of $G(x) / \rho$ for 1 -cm-radius metal spheres levitated at a constant skin depth of $1 / 4 \mathrm{~cm}$.

Chart 6 gives a near-optimum value of the frequency for the levitation of metal spheres of 1 - cm radius. This frequency is calculated from Eq. (5)

$$
x=R\left(\frac{1}{2} \omega \mu \sigma\right)^{1 / 2}
$$

with the assumptions that $\mathrm{x}=4.0, \mathrm{R}=1 \mathrm{~cm}$, and the densities are those given in Chart 1.

Charts 7 through 12 give the same set of data and calculations for the liquid or solid elements at either their respective melting points or the temperatures given. If no value of the density of the liquid metal is available, the density of the solid at $20^{\circ} \mathrm{C}$ is used. Since the data are less plentiful and precise at these temperatures, the quantities given in these charts must be used with caution. The dramatic change in the levitation properties of the 


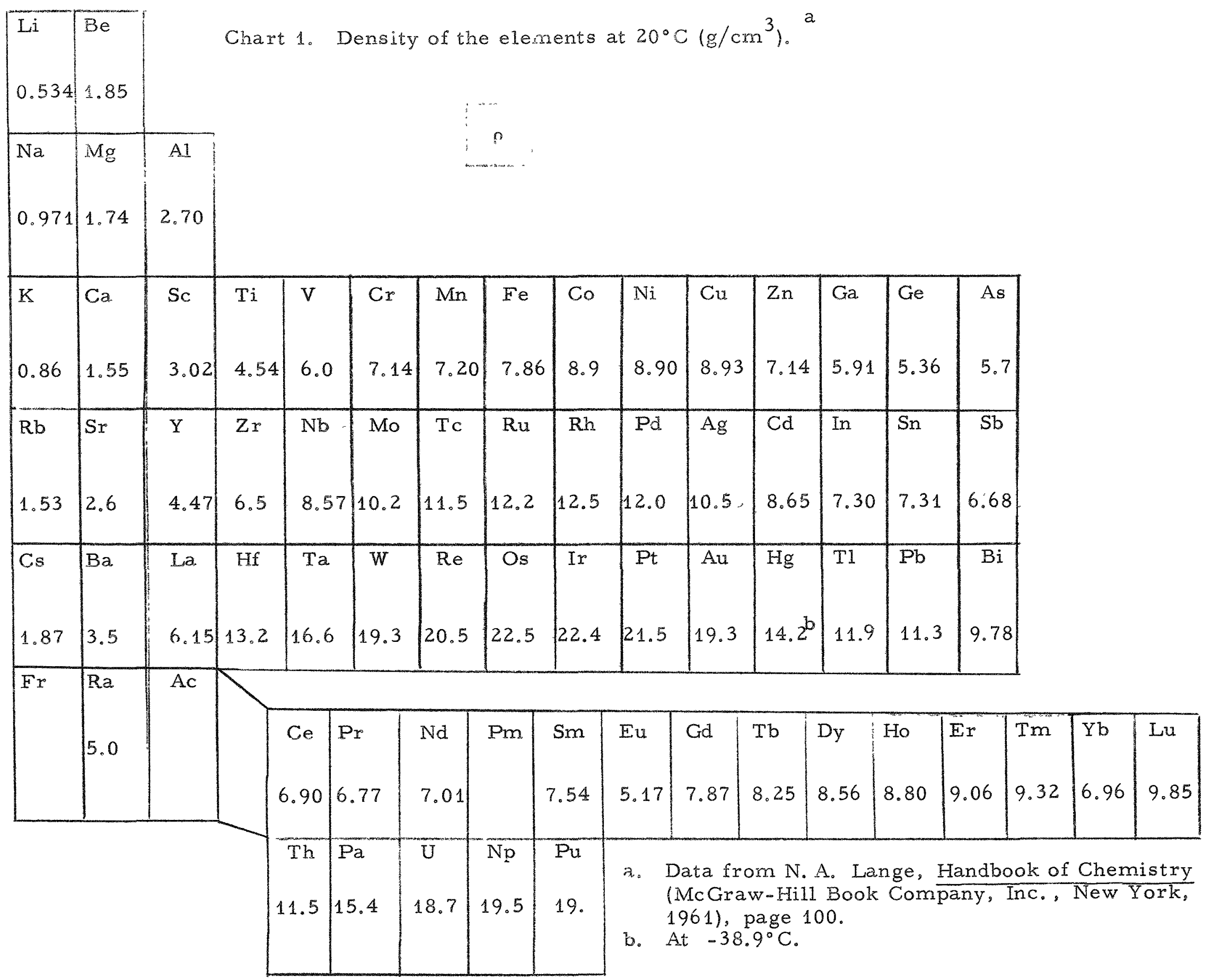




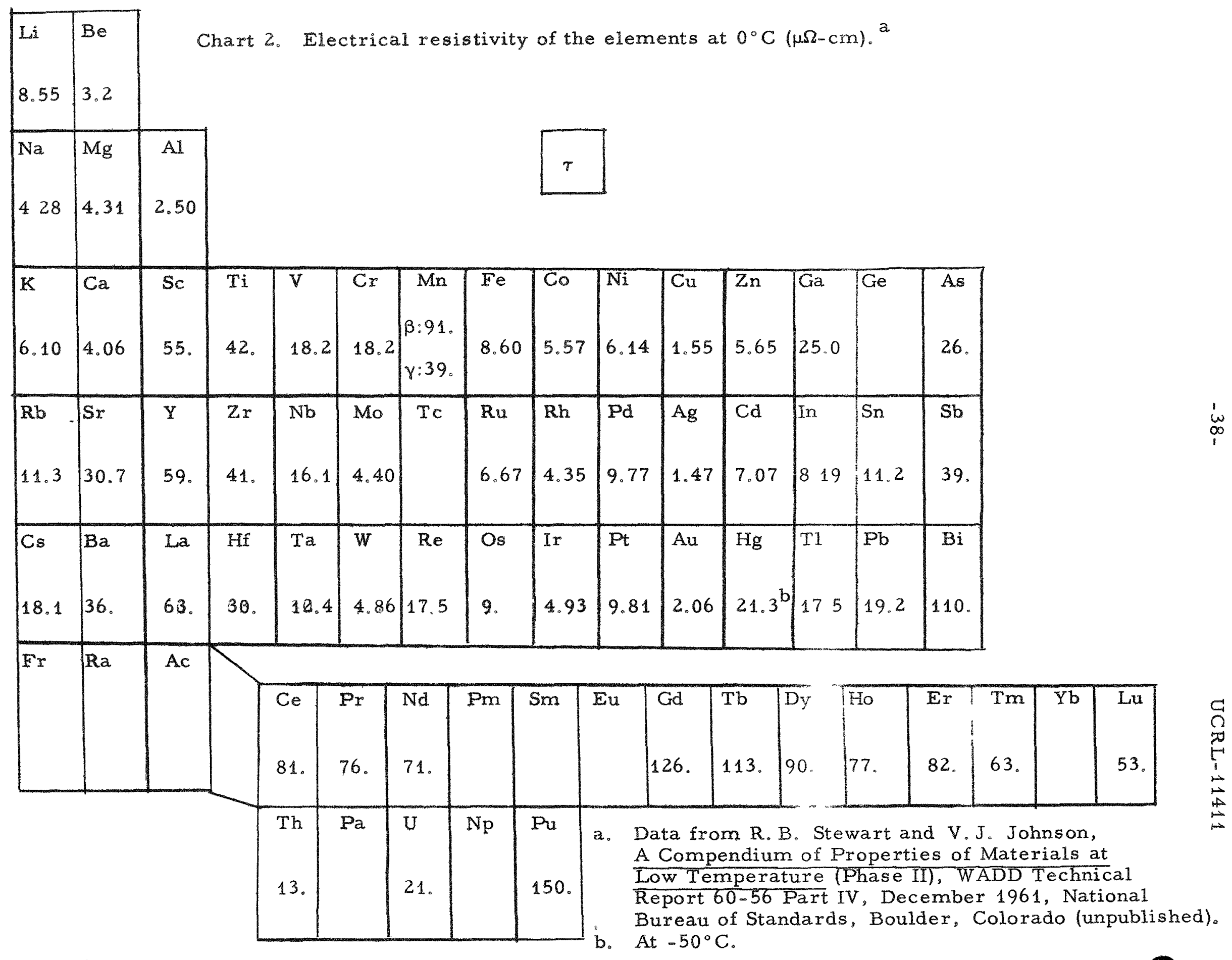




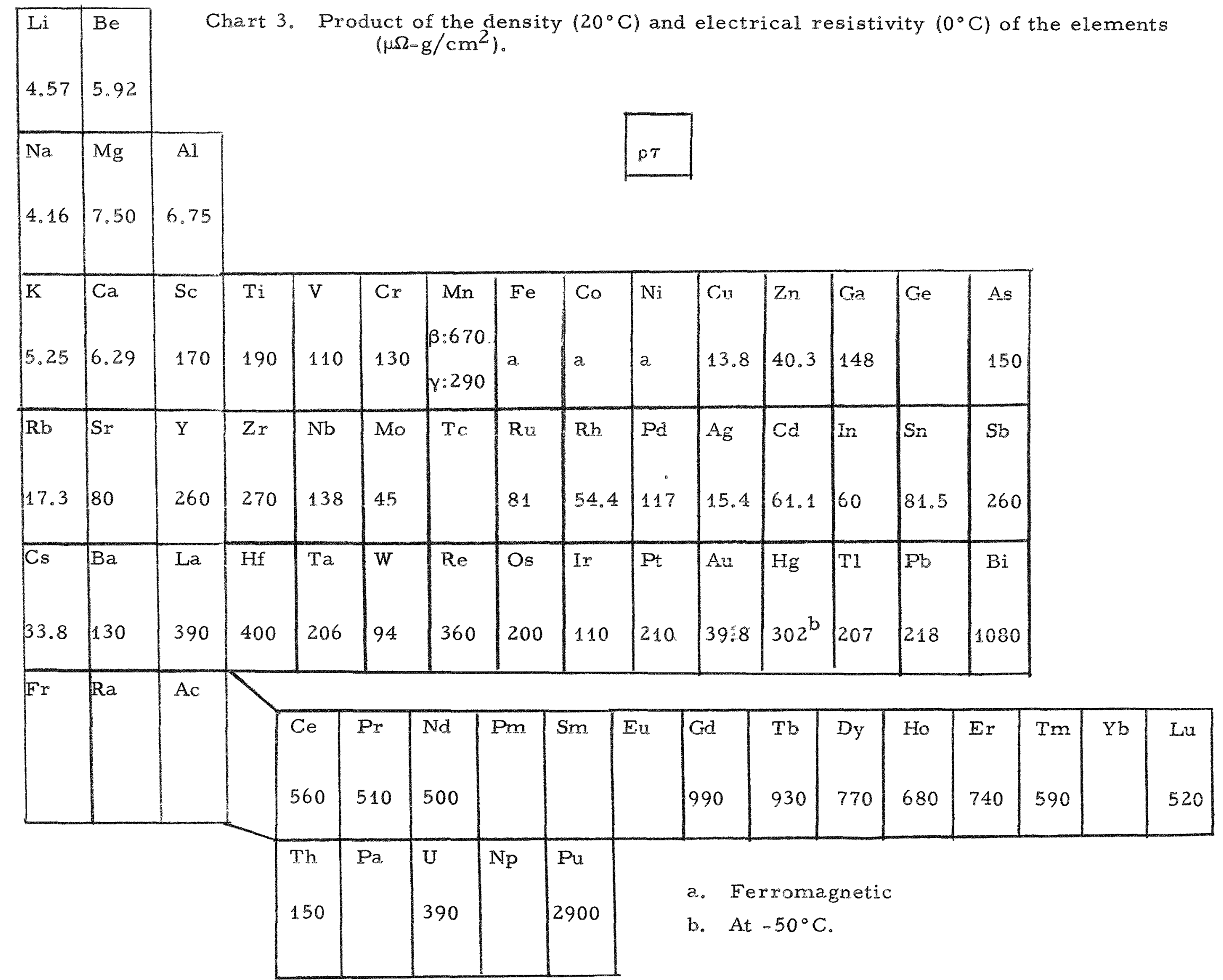


elements at higher temperatures is well illustrated by these charts, even if they are accurate only to within 10 or $20 \%$ 。

Figures 17 and 18 correspond to Case 1 in Sec. III.C., summarizing pictorially the data for the levitation of some common metals at a constant skin depth and radius. Figures 19 and 20 correspond to Case 2 , levitation at a constant frequency, $10 \mathrm{kc} / \mathrm{sec}$. Terms not involving $\omega, \tau, \rho$, or $\mathrm{R}$ have been eliminated from Eqs. (3) and (4), leaving

$$
\begin{array}{ll}
\text { Force } \propto G(x) & \text { (dimensionless) } \\
\text { Force/weight } \propto \frac{G(x)}{p} & \left(\mathrm{~cm}^{3} / g\right) \\
\text { Power } \propto \frac{p T}{F(x)} & \left(\mu \Omega-g / \mathrm{cm}^{2}\right) .
\end{array}
$$

This is the explanation for the unusual units given for the quantities at the bottom of the columns. It is apparent that density plays an extremely important role in levitation phenomena, accounting for the fact that the alkali and alkaline earth metals, and aluminum, are the easiest to levitate.

Figure 21 can be conveniently used to determine values of $x$ if $\sigma, v$, and $R$ are known.

\section{Magnitude of the Magnetic Field}

With the sole exception of Hulsey's, ${ }^{20}$ no measurements have been made of the strength of the magnetic field for levitation-coil shapes and designs currently used. Even he measured only the magnetic-field strength for a 3-in.-diam standard solenoid. Fortunately, the analytical solutions for a circular loop can be used with good accuracy, and only the diameter, the relative location of each loop of the multi-turn coil, and the rms current are needed to calculate the rms field strength.

The minimum field needed to levitate a $1 / 2-\mathrm{cm}$-diam sphere of aluminum or beryllium is about 200 Gauss. Typical values for coils used by 

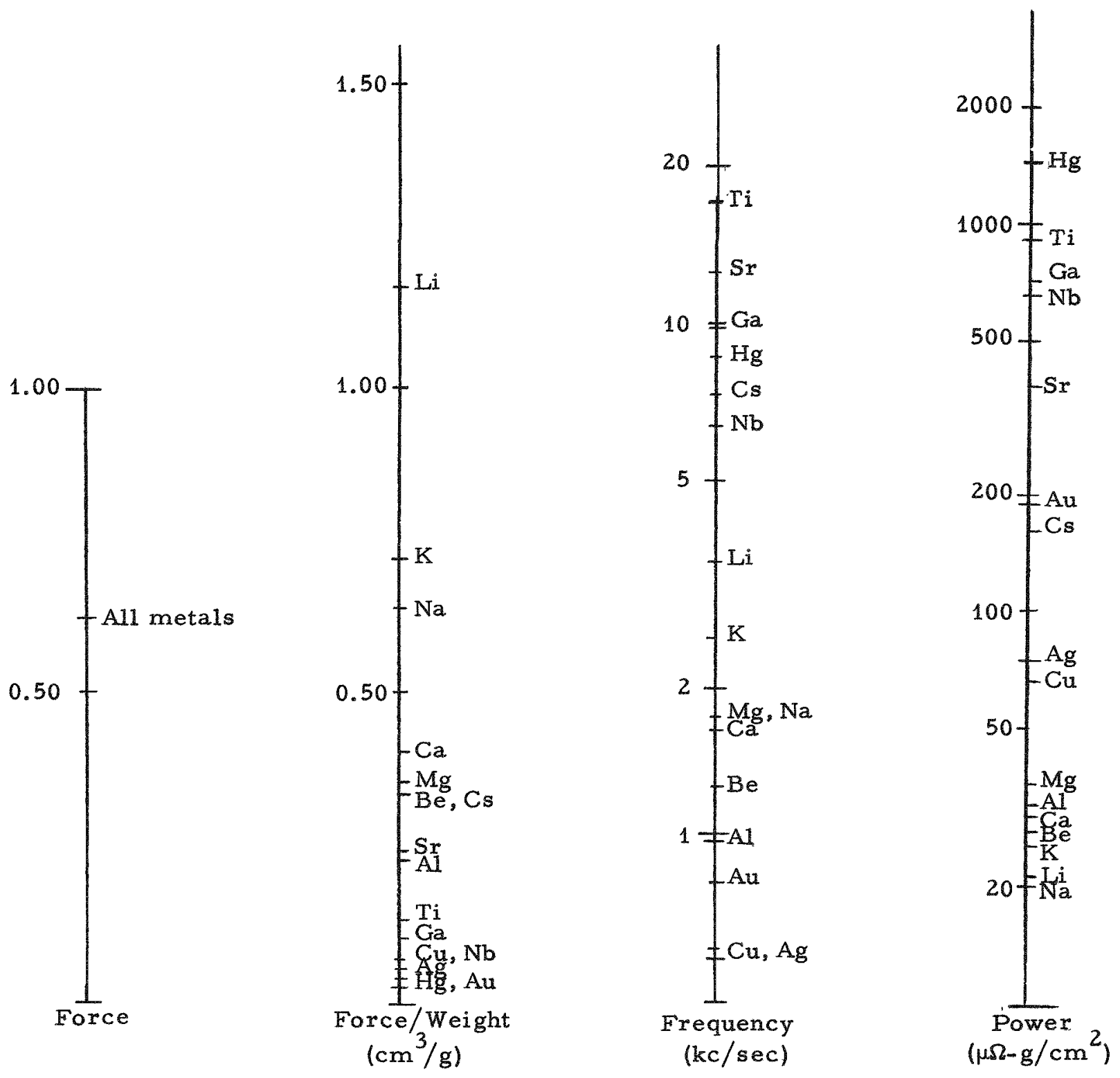

Fig. 17. Levitation at constant $\mathrm{skin}$ depth and radius with $\mathrm{x}=4.0$ and $R=1 \mathrm{~cm}$. Data is from Charts 1 through 6 . 


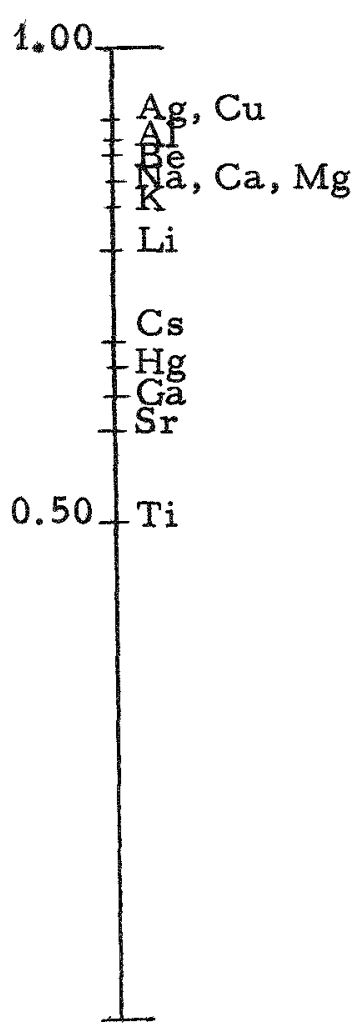

Force

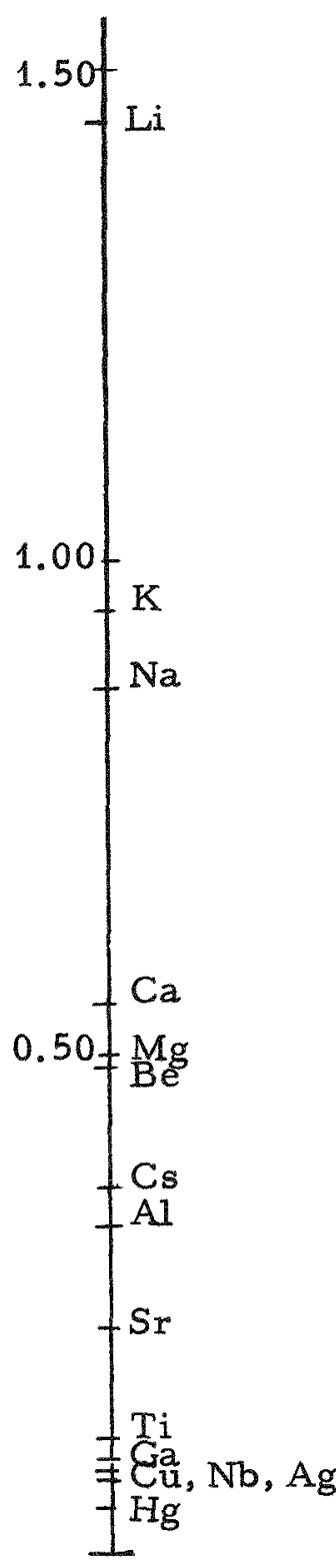

Force/Weight $\left(\mathrm{cm}^{3} / \mathrm{g}\right)$

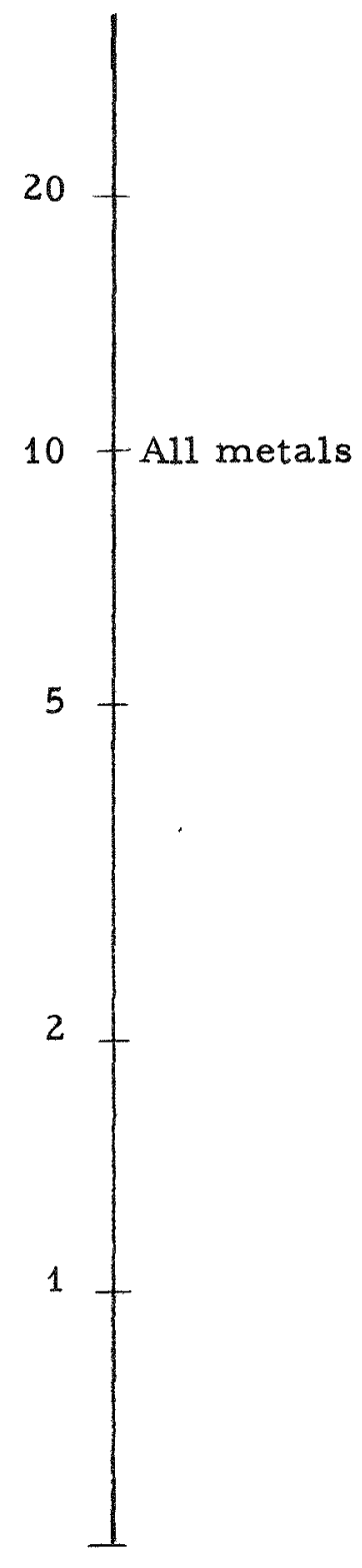

Frequency
$(\mathrm{kc} / \mathrm{sec})$

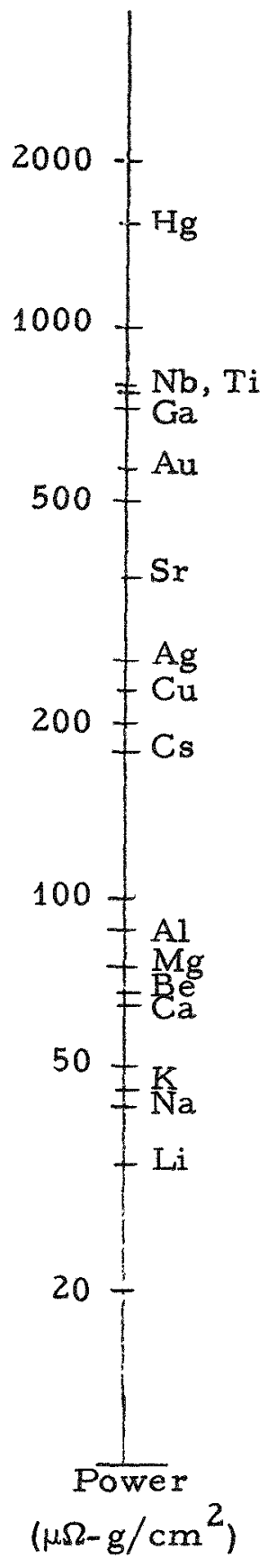

Fig. 18. Levitation at constant frequency and radius with $\nu=10 \mathrm{kc}$ and $R=1 \mathrm{~cm}$. Data from Charts 1 through 6 . 

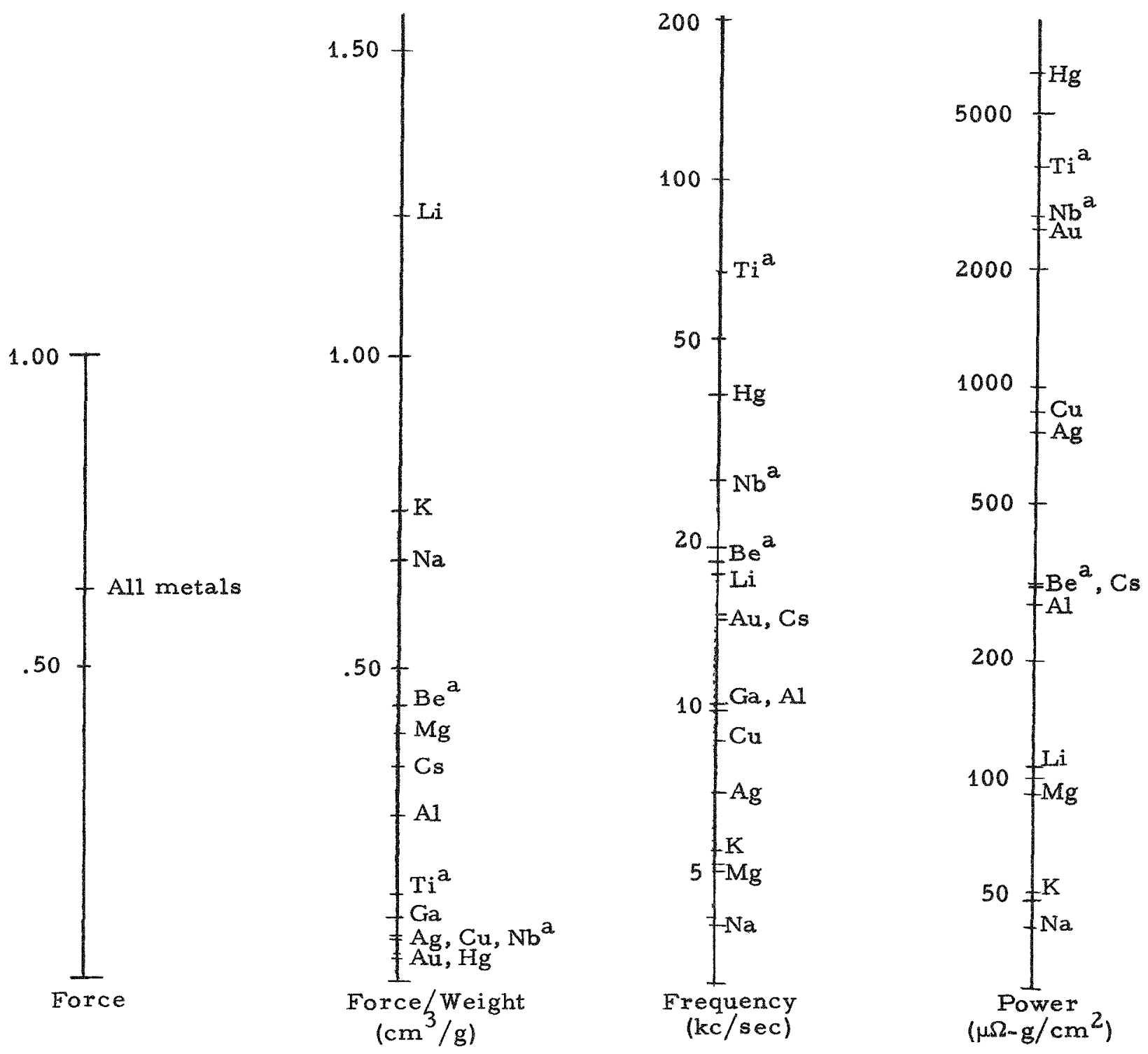

a. solid

Fig. 19. Levitation at constant skin depth and radius with $x=4.0$ and $\mathrm{R}=1 \mathrm{~cm}$. Data is from Charts 7 through 12 . 


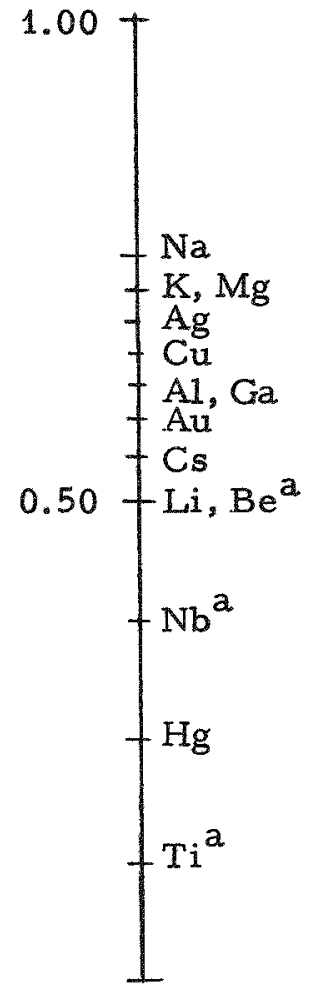

Force
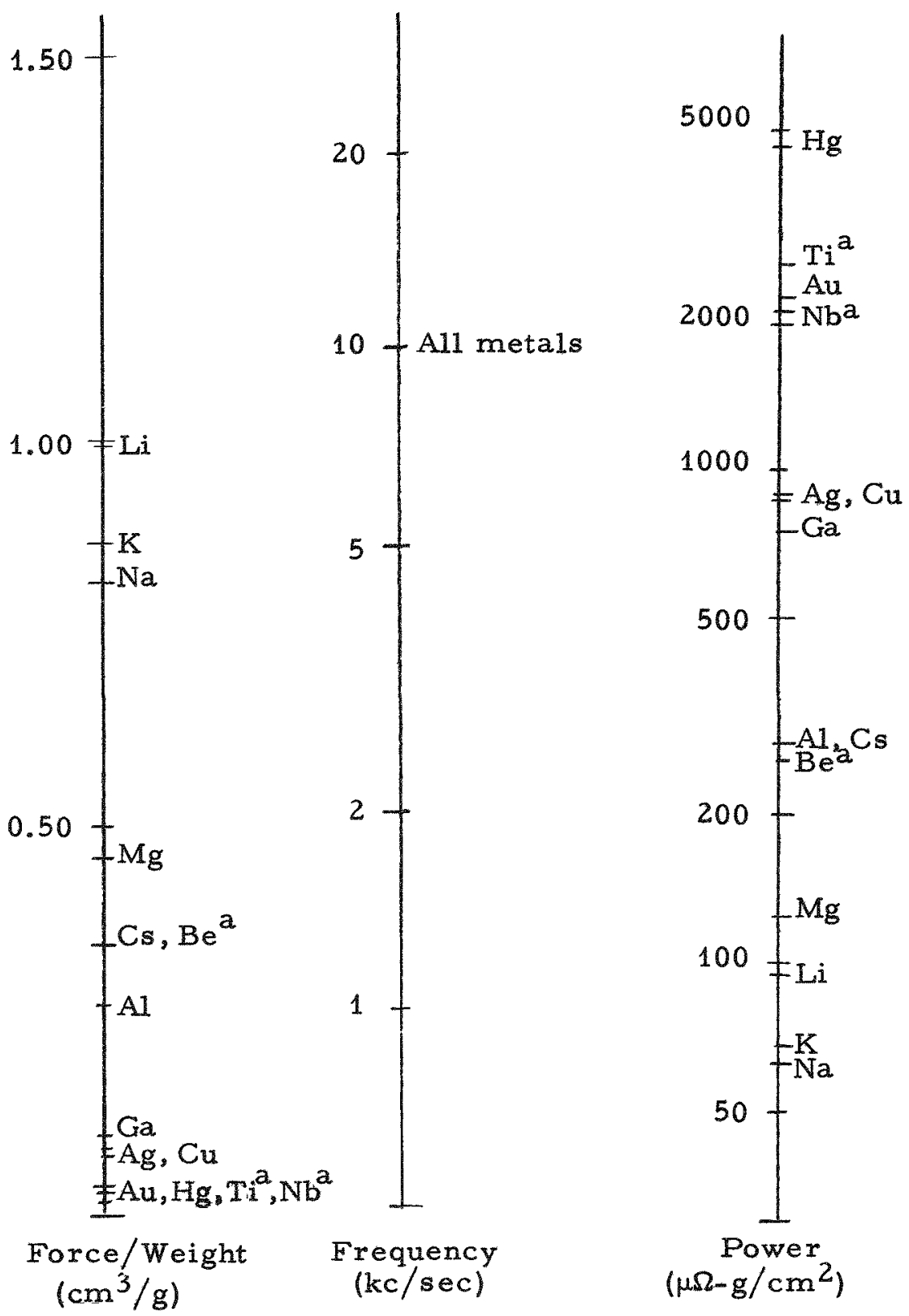

a. solid

Fig. 20. Levitation at constant frequency and radius with $R=1 \mathrm{~cm}$. Data is from Charts 7 through 12. 


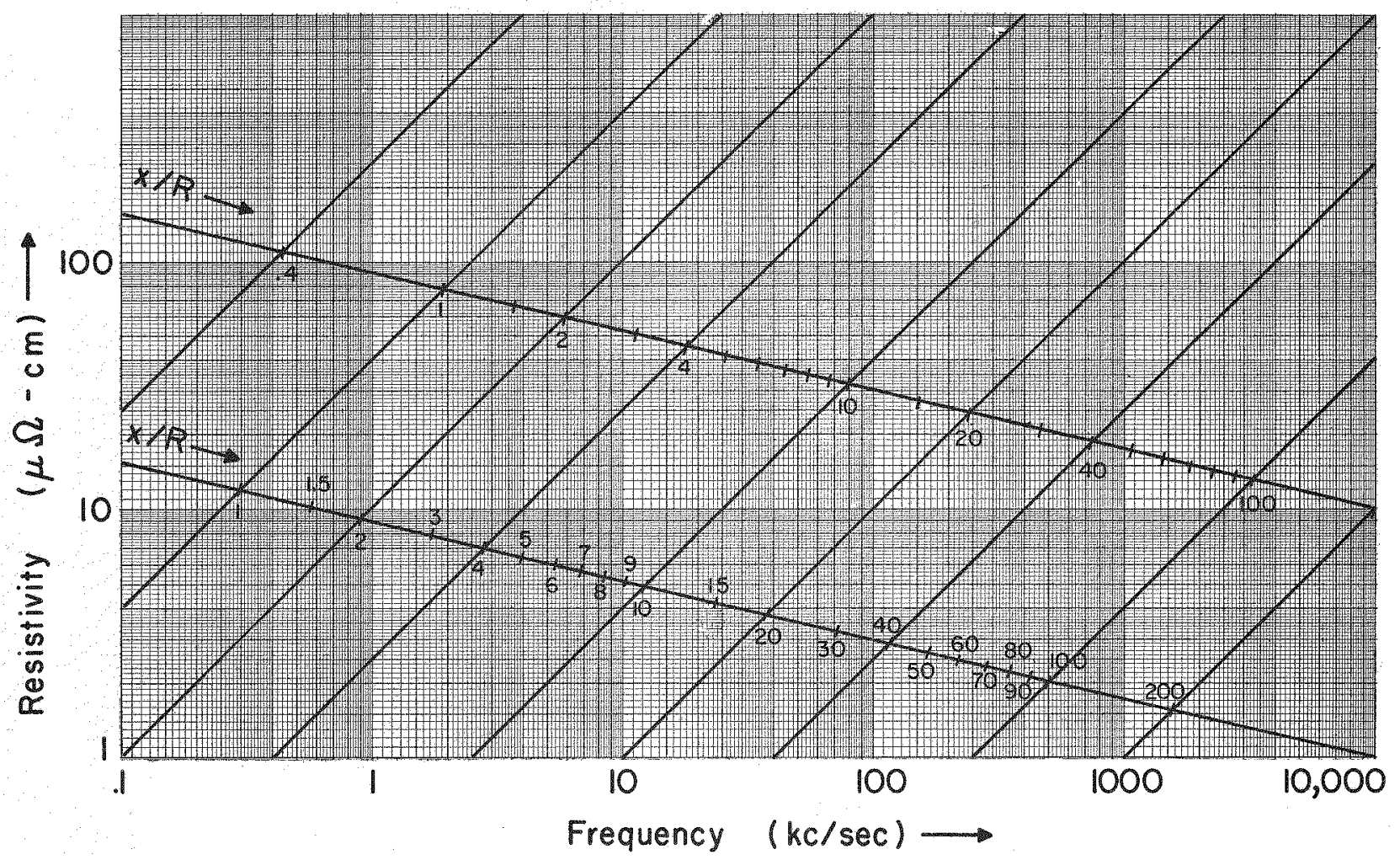

MUB - 3039

Fig. 21. Representation of parameter $x$ for the evaluation of the dimensionless functions $G(x), H(x)$, and $F(x)$. 


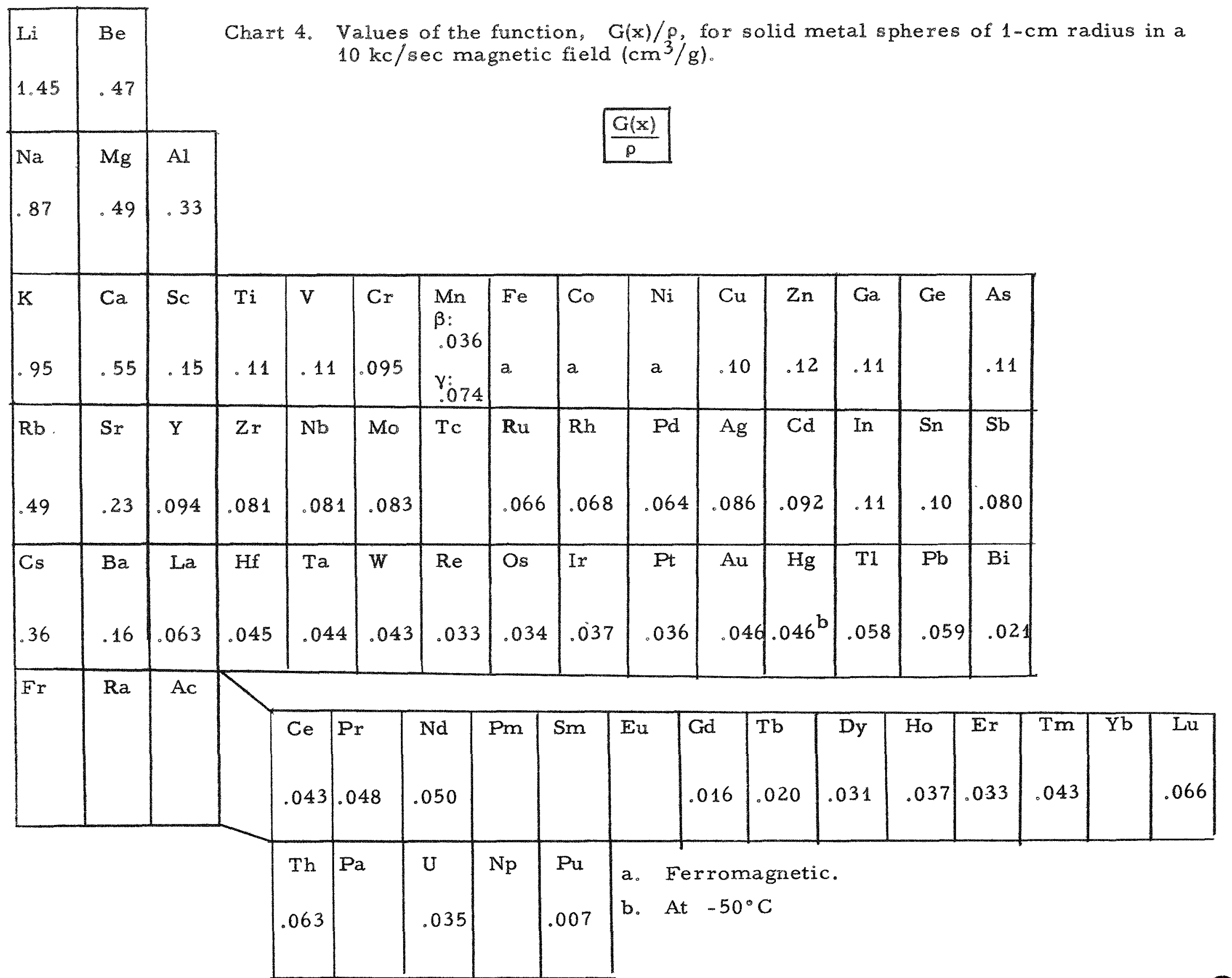




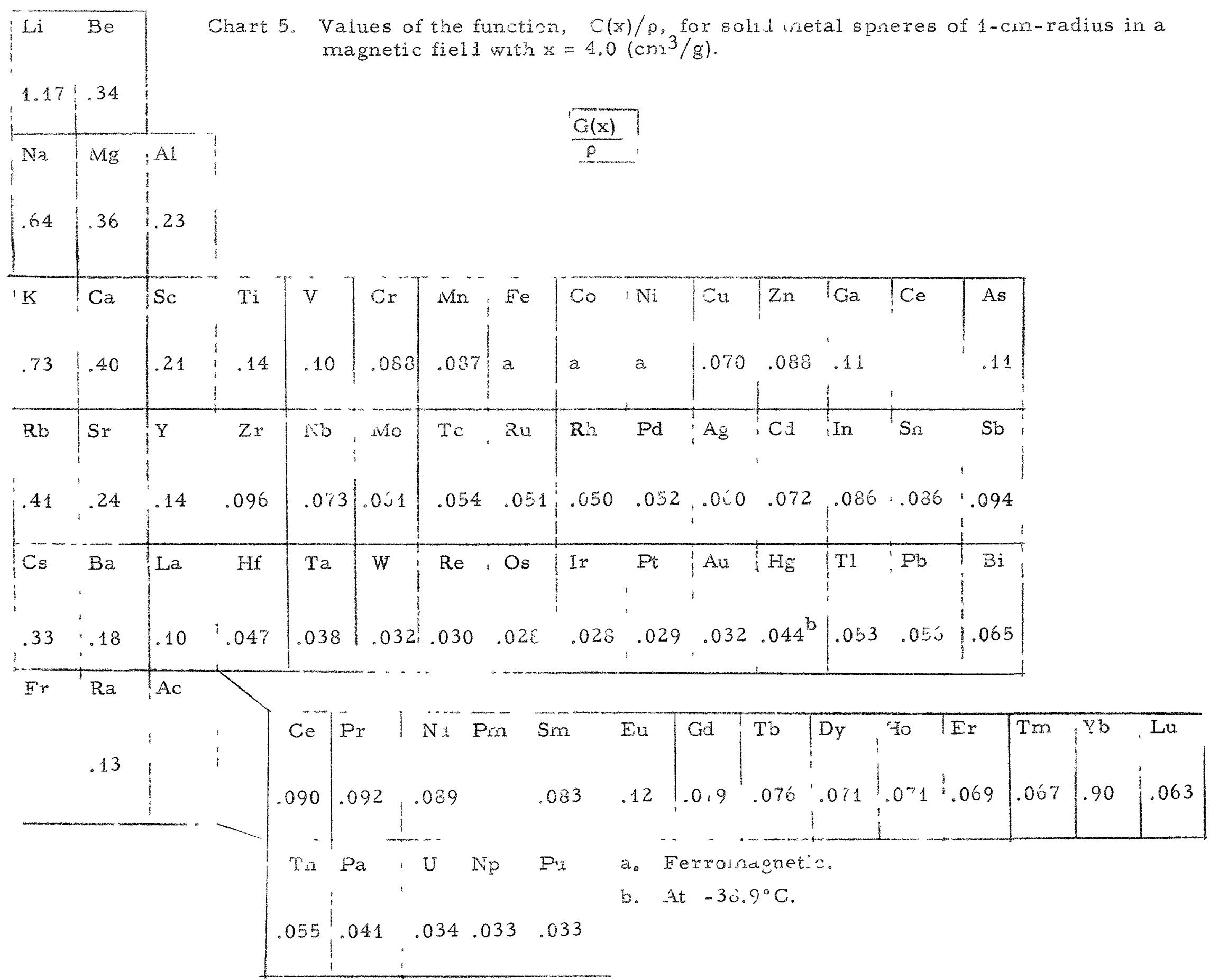




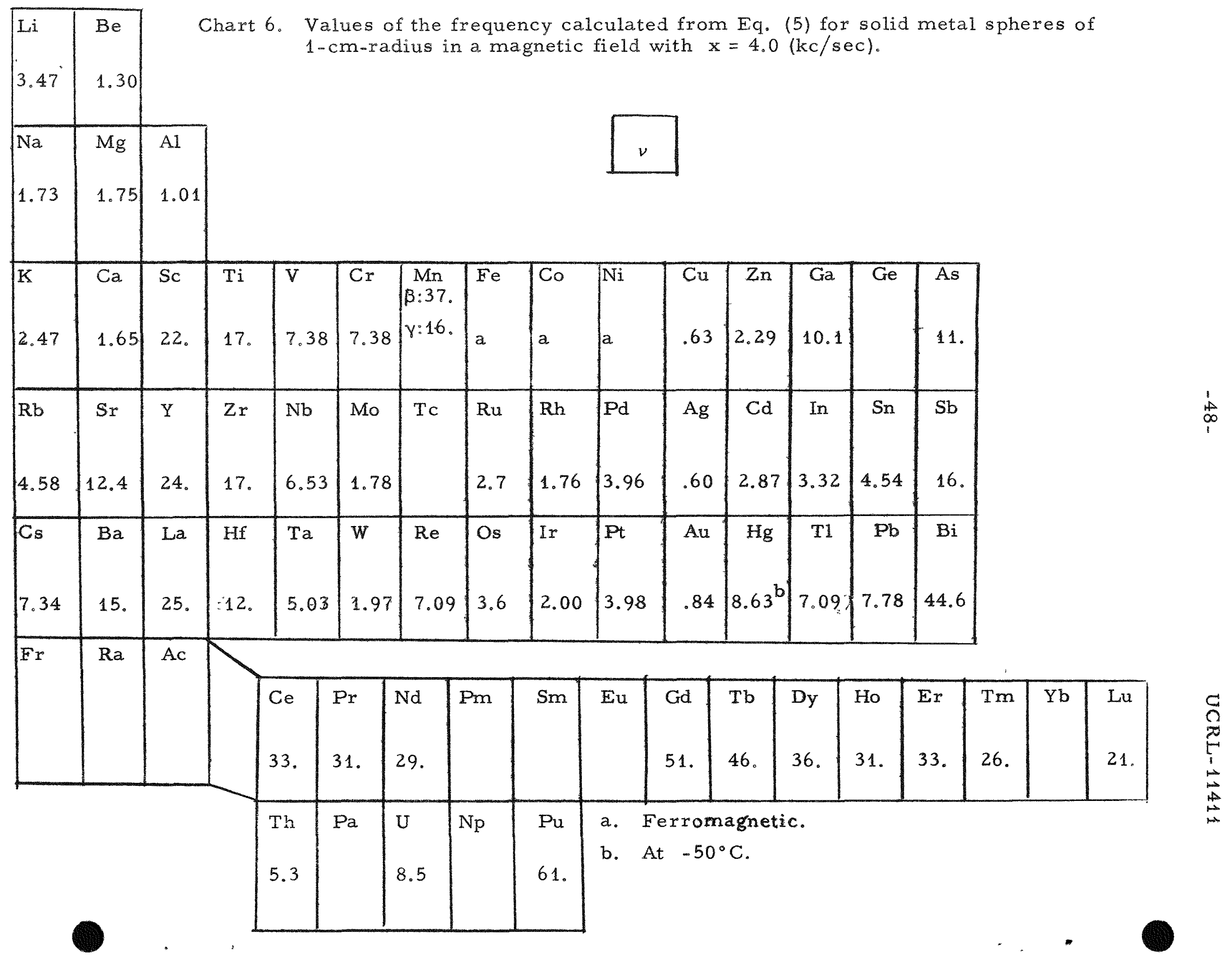




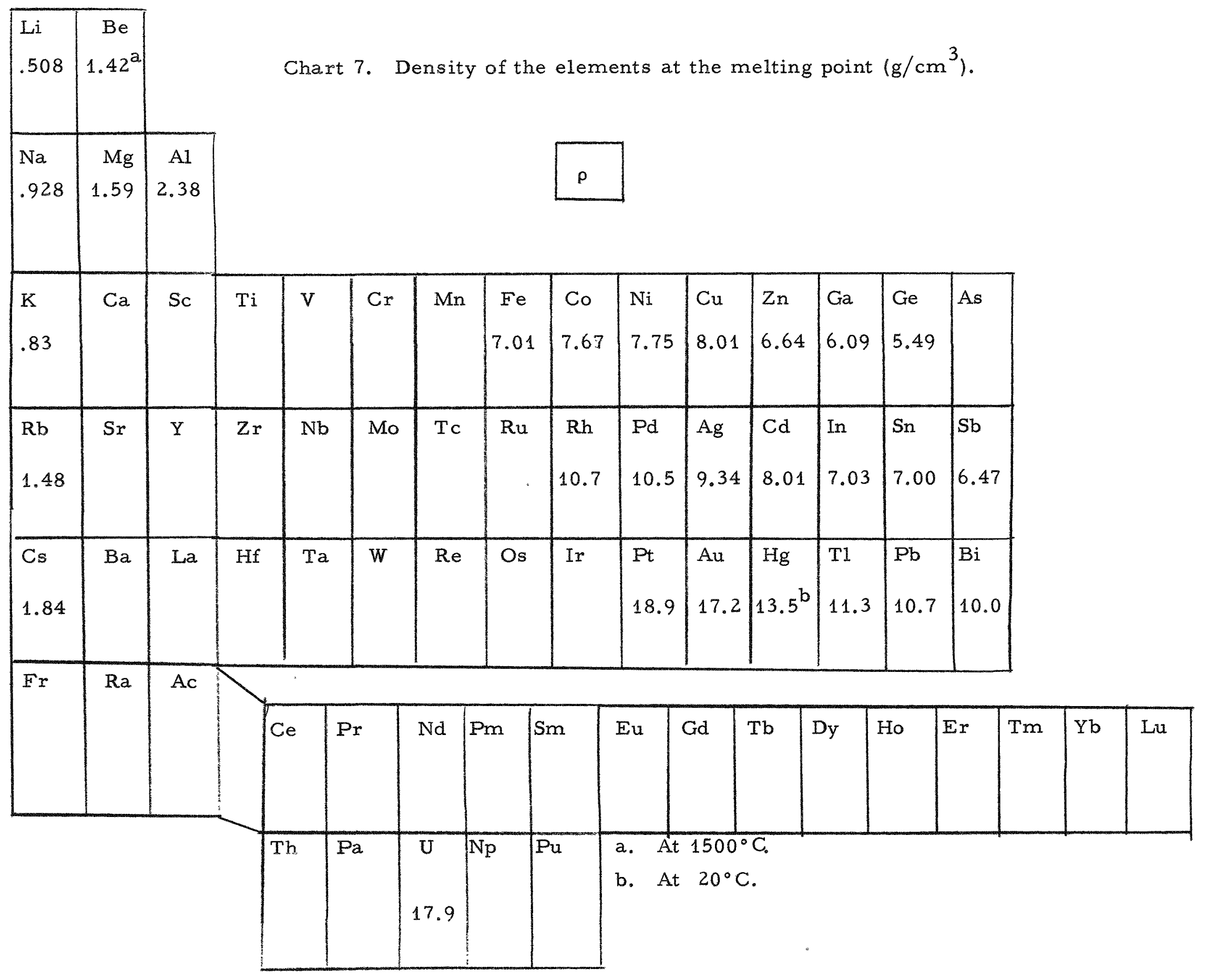




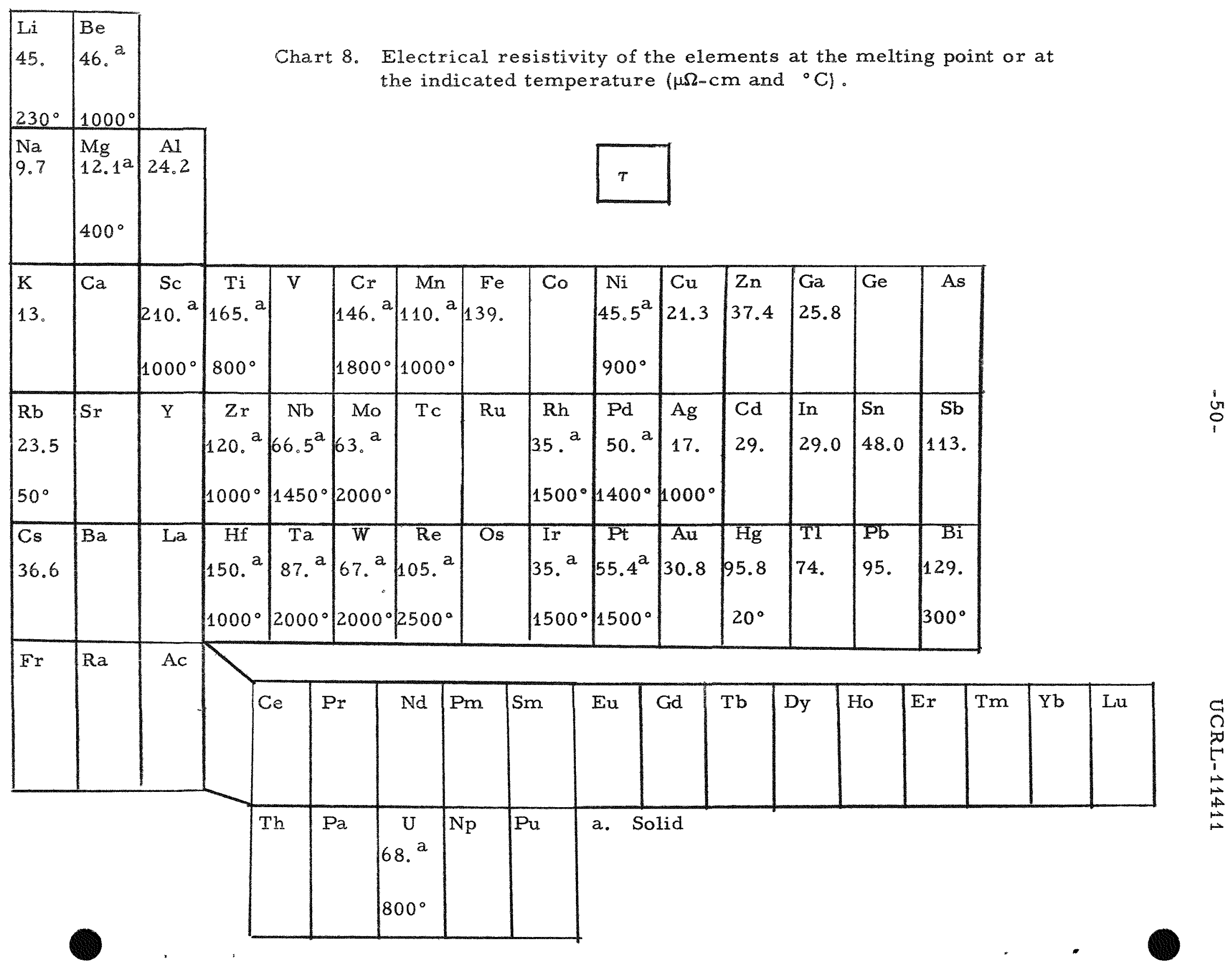




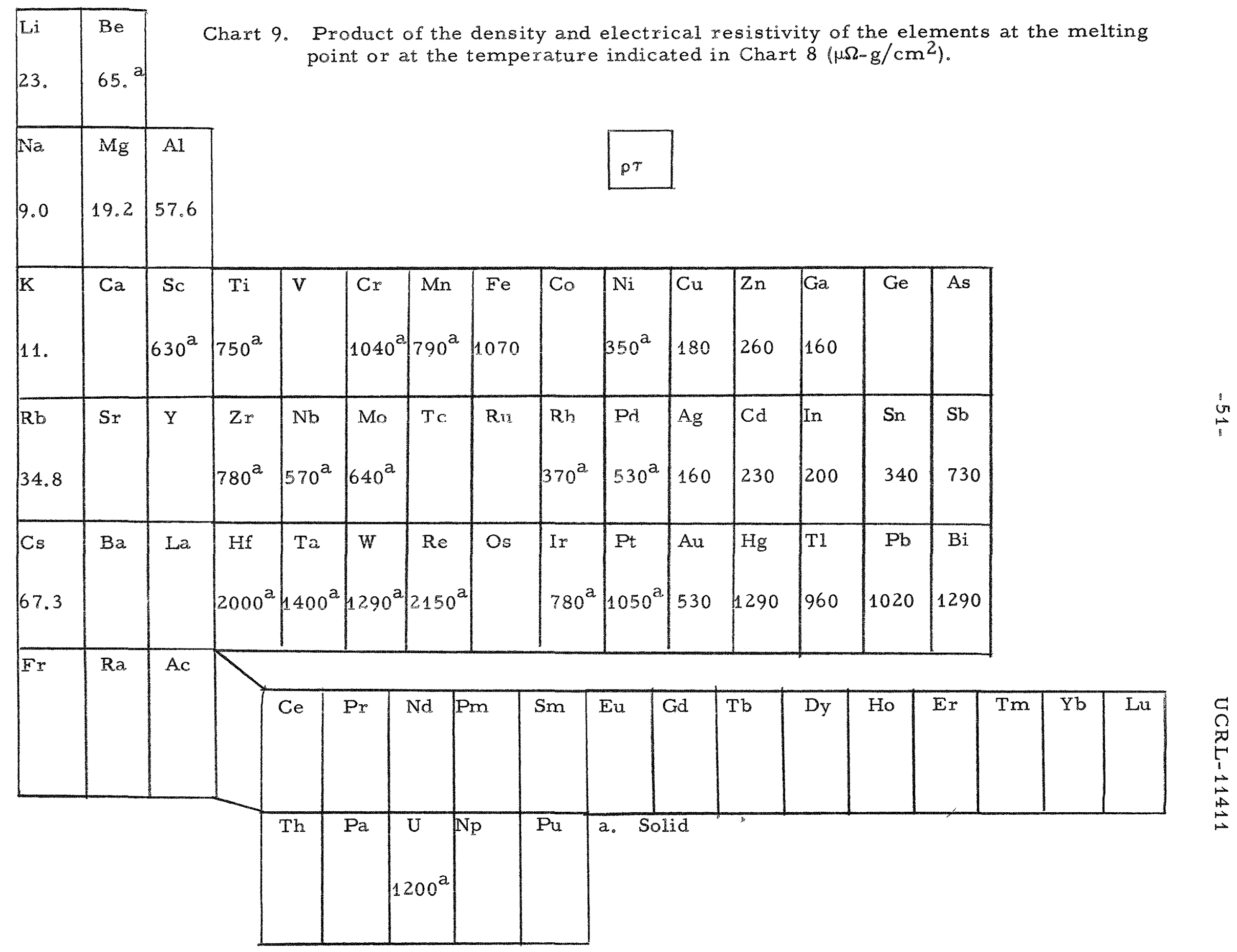




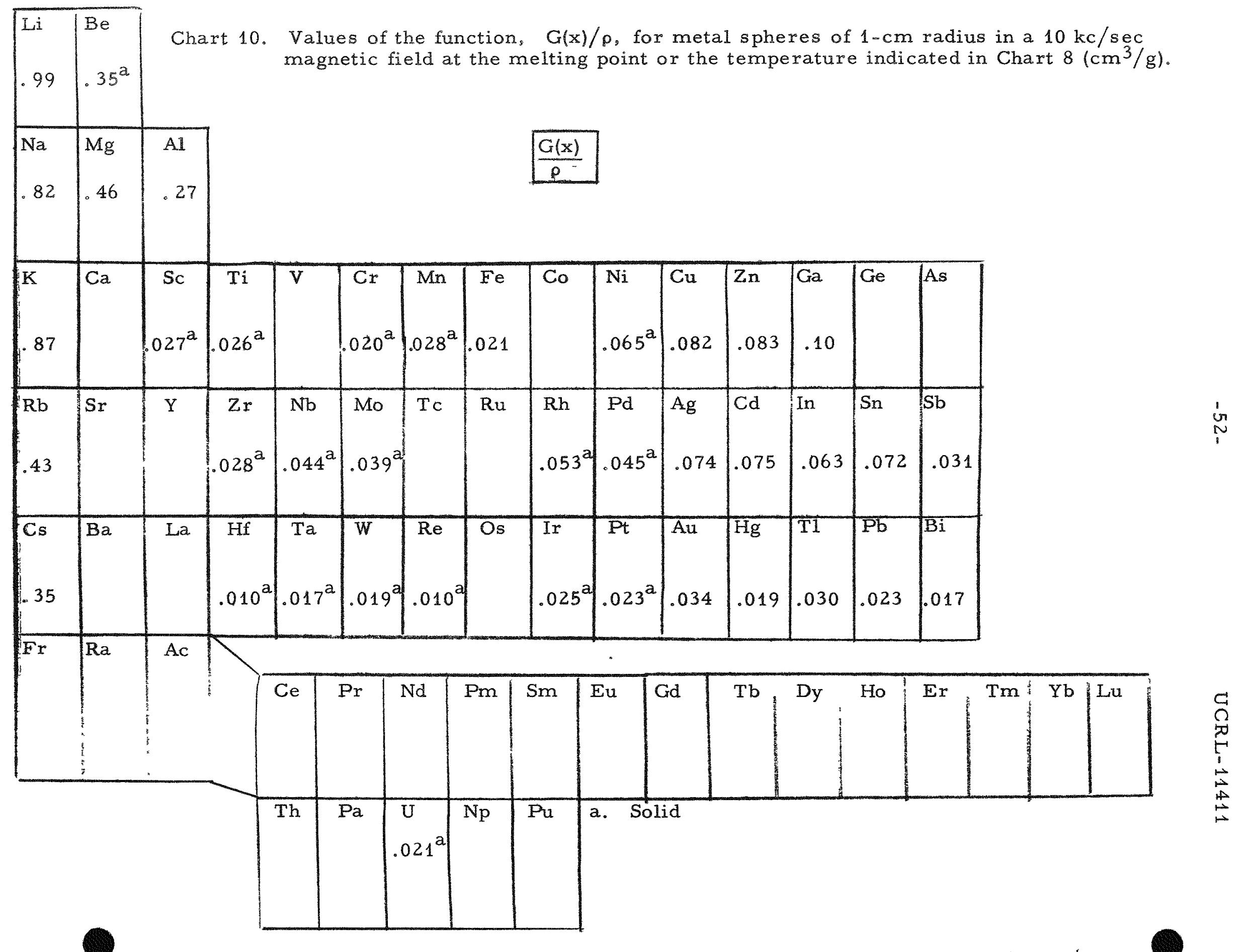




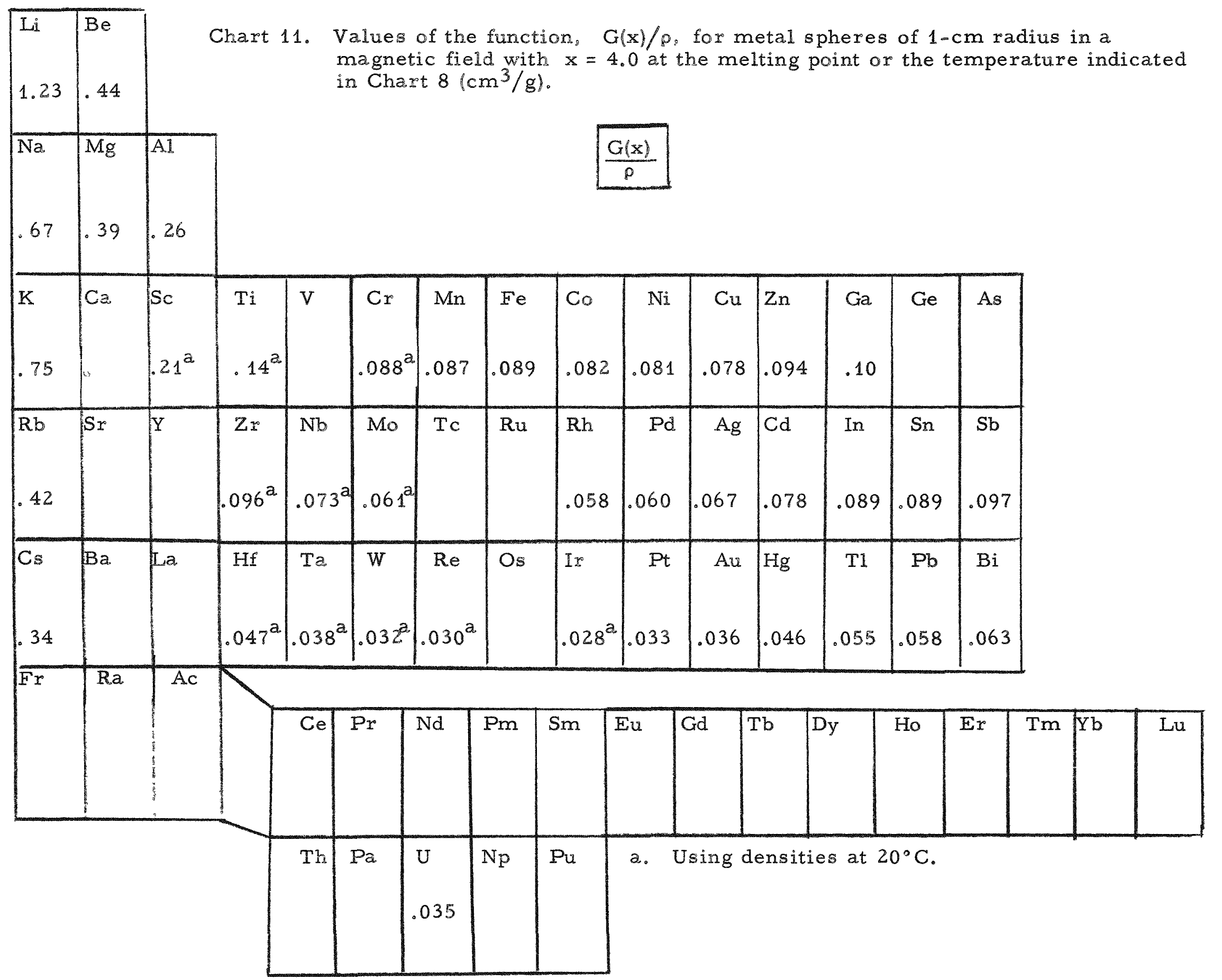




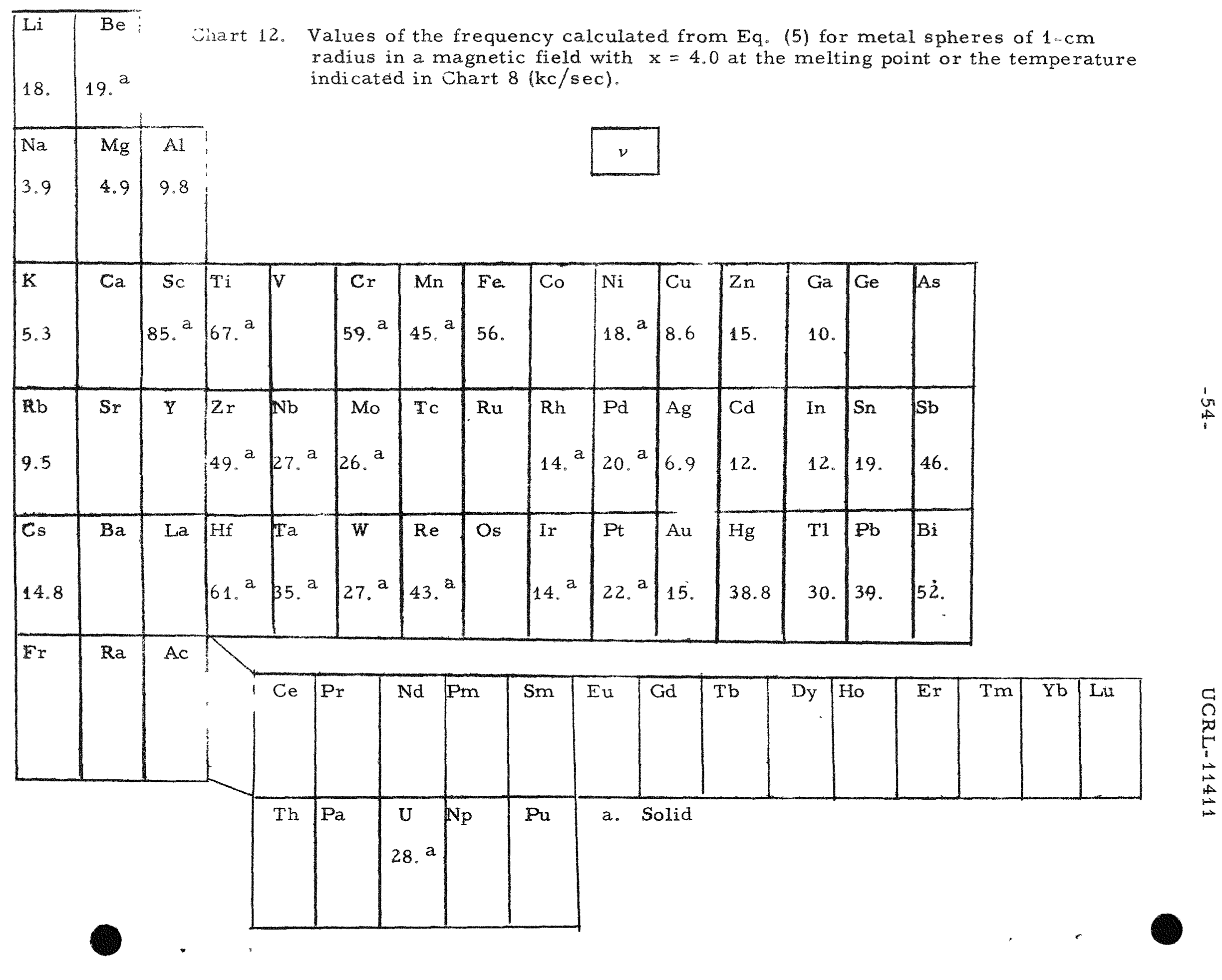


Comenetz and Salatka, ${ }^{9}$ Hulsey, ${ }^{20}$ Jenkins, ${ }^{21}$ Polonis et al., ${ }^{24,25}$ Begley et al., ${ }^{7}$ and Weisberg 26 are probably in the 250 to $500 \mathrm{G}$ range. Okress et a1. 19,27 appear to have used as much as $700 \mathrm{G}$ in their experiments. From Hulsey's calculations, typical field gradients are of the order of 50 to $500 \mathrm{G} / \mathrm{cm}$, depending upon the number of turns, their diameter, and their spatial distribution, as well as the magnitude of the coil current.

The exact magnitude of the field and field gradient near the levitated metal depends on its exact position within the magnetic field where it is levitated. Thus, the figures given above must be used with caution. Nevertheless, they do give the correct order of magnitude for the quantities involved.

Chart 13 gives the values of the magnetic field required to levitate $1-\mathrm{cm} \sim$ radius spheres of the solid metal elements at $0^{\circ} \mathrm{C}$, as calculated from Eq. (49),

$$
R=\frac{B^{2}}{4 \rho g \mu_{0}}
$$

This equation is the result of integrating the magnetic pressure normal to the metal surface over the lower hemisphere area and then equating the force obtained to the total weight of the sphere. The values obtained in Chart 13 are extremely reasonable. They are most valid for conditions in which the skin depth is much less than the sphere radius, i.e., at high frequencies.

An ac gauss meter, which reads as much as $3000 \mathrm{G}$ at frequencies up to $30 \mathrm{kc} / \mathrm{sec}^{28}$ should be of considerable use in determining whether a given inhomogeneous magnetic field can levitate a chosen metal sample. 


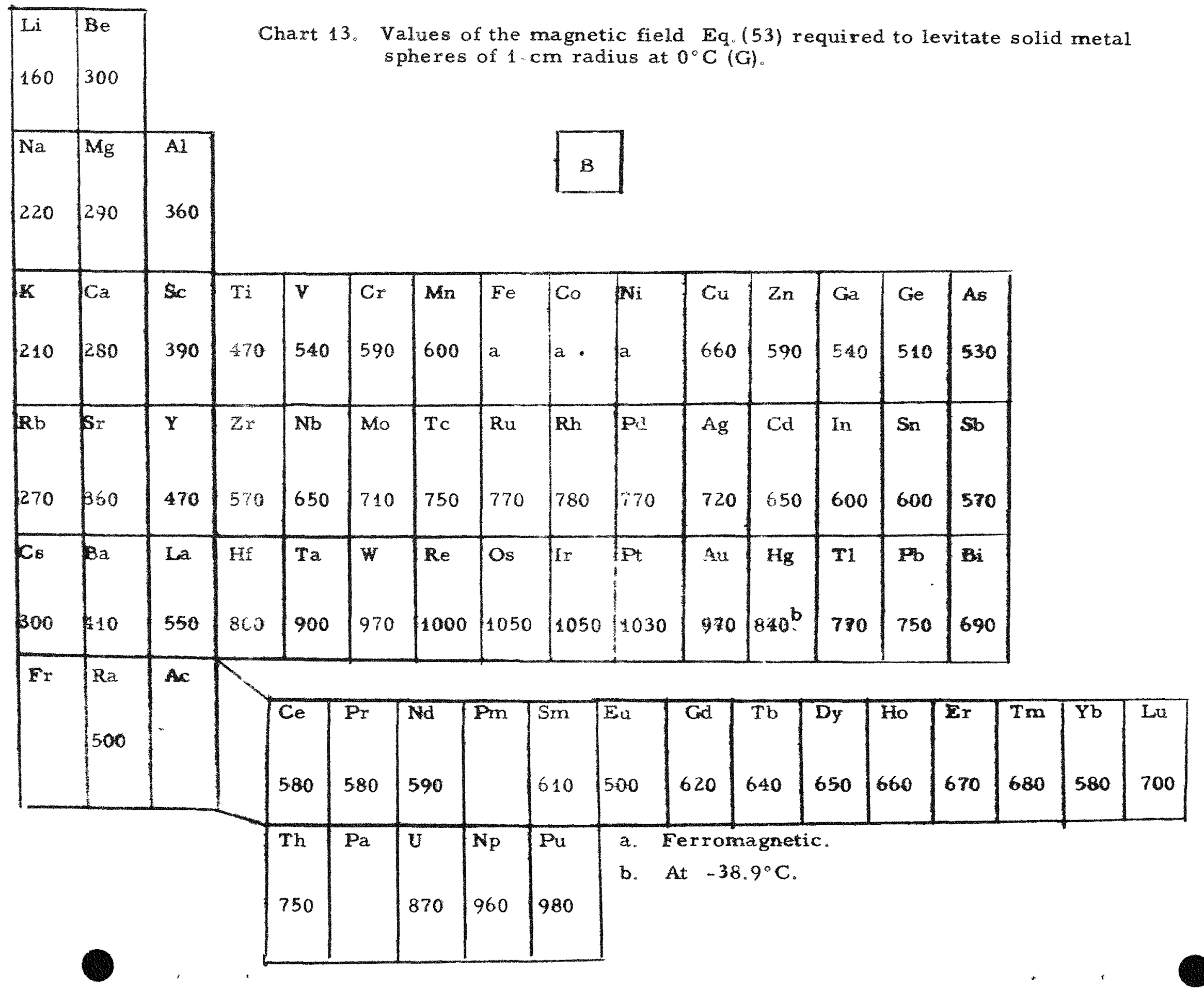


IV. THEORY OF THE ELECTROMAGNETIC LEVITATION OF LIQUID METALS

All of the previously derived equations do not apply to the case of determining the stability of a nonrigid levitated material such as a liquid metal. In such a case, although Eq. (3) gives general guidelines, a more detailed description of the point-to-point variation in the gravitational, surface tension and magnetic "pressures" over the surface of the liquid sample is required.

Consider a liquid-metal sample levitated in an alternating magnetic field with a field distribution such that the metal assumes a completely sphexical shape. It is assumed that the skin depth is much less than the sphere radius. Under these conditions [according to Colgate et al。 ${ }^{29}$ ] "a mean hydrostatic pressure $\mathrm{B}^{2} / 2 \mu_{0}$ is exerted normal to the metal surface." The gravitational, surface tension, and magnetic pressures exerted on the liquid metal at the lowest point of the sphere are

$$
\begin{aligned}
& P_{g}=g h\left(\rho-\rho_{0}\right) \\
& P_{s}=\frac{2 \gamma}{R} \\
& P_{m}=\frac{B_{\text {bottom }}^{2}-B_{\text {top }}^{2}}{2 \mu_{0}}
\end{aligned}
$$

In the absence of electromagnetic forces, the radius of the sphere when the gravitational and surface tension pressures are approximately equal to each other is given by the formula

$$
\mathrm{R}^{2}=\frac{\gamma}{\rho \mathrm{g}} \text {. }
$$

In the absence of surface-tension forces, the radius of the sphere when the gravitational and magnetic pressures approximately balance each other is calculated below with the as sumption that there is only a small field at the top 
of the sphere,

$$
R=\frac{B^{2}}{4 \rho g \mu_{0}}
$$

where $\rho$ is the metal density, $\rho_{C}$ is the density of tic external dielectric mediurs, $\gamma$ is the surface tension, $B$ is i'ze rms rnagnelic-field strength, and $g$ is the gravitational constant (approximately $980 \mathrm{~cm} / \mathrm{sec}^{2}$. In vacuums, $\rho_{0}=0$. In these approximations, the heignt of the liquid-metal column is chosen to be equal to $2 R$.

The values of the surface tensions of the liquid elements at their respective melting points is given in Chart 14. The data have been obtained from the Handbook of Chemistry and Physics, ${ }^{30}$.Iandbook of Chemistry, 31 Metals Reference Book, ${ }^{22}$ recent references in Chenical Abstract, Mayer, 32 Taylor, ${ }^{33}$ or from the empirical formula of Strauss, 34,35 with neat of vaporization data obtained from Brewer. 36

Chart 15 summarizes the values of the radii calculated from Eqs. (52) and (53). The top number in each element box corresponds to Eq. (52), whereas the bottom one is calculated from Eq. (53) with a value of $1 \mathrm{kG}$ assumed for the field strength. The density used in these calculations is that for the solid metal elements at $20^{\circ} \mathrm{C}$, a.value from $2 \%$ to $15 \%$ higher than the actual density of the liquid metals at their respective melting points. No great accuracy is claimed for the figures given in Chart 15. The numbers semiquantitatively indicate how the metals differ from each other.

Unfortunately, these calculations are naive and may not even come close to predicting the true amount of a particular metal that can be levitate 1 in the liquid state. The flaw in the arguments given above is that, to deter mine the stability of the levitated metal system, both the static and dynamic aspects must be considered. Static stability is concerned with the production 
of restoring forces and corresponds to the above considerations, for which the gravitational, surface tension, and electromagnetic forces are balanced. Dynamic stability is concerned with the oscillations that are set up in the system as a result of these restoring forces. If these oscillations are damped, then the system is dynamically stable. If they are divergent, then the system is dynamically unstable. Dynamic instability may occur with either static stability or static instability。

If the levitated metal is dynamically unstable, the existence of this condition can be noticed almost immediately; the metal may separate into smaller liquid drops, oscillate violently, or fly apart. This condition should not be confused with the effects mentioned in Sec. III. B, where, as the metal gradually increases in temperature, the force decreases until the metal no longer can be sustained by the field. According to Colgate, "29 "the presence of appreciable surface tension may be expected to inhibit the growth of instabilities, especially those having short wavelengths. "This fact explains why stable levitation of liquid-metal samples of moderate size can be achieved.

The exact criteria for dynamic stability, of a levitated system, in volving factors such as the metal size and shape, skin depth, magnetic field distribution and intensity, density, and surface tension, have not yet been explicitly stated. The calculation of these criteria will involve many of the techniques now used to determine the stability of high-energy plasmas in controlled-nuclear-fusion research. The analogy between the two physical problems is quite close. It can even be speculated that some of the techniques used to stabilize and confine gaseous plasmas, such as the application of a static magnetic field in a suitable direction, may be applicable to metal levitation. The possibility of such an approach awaits further calculations. 


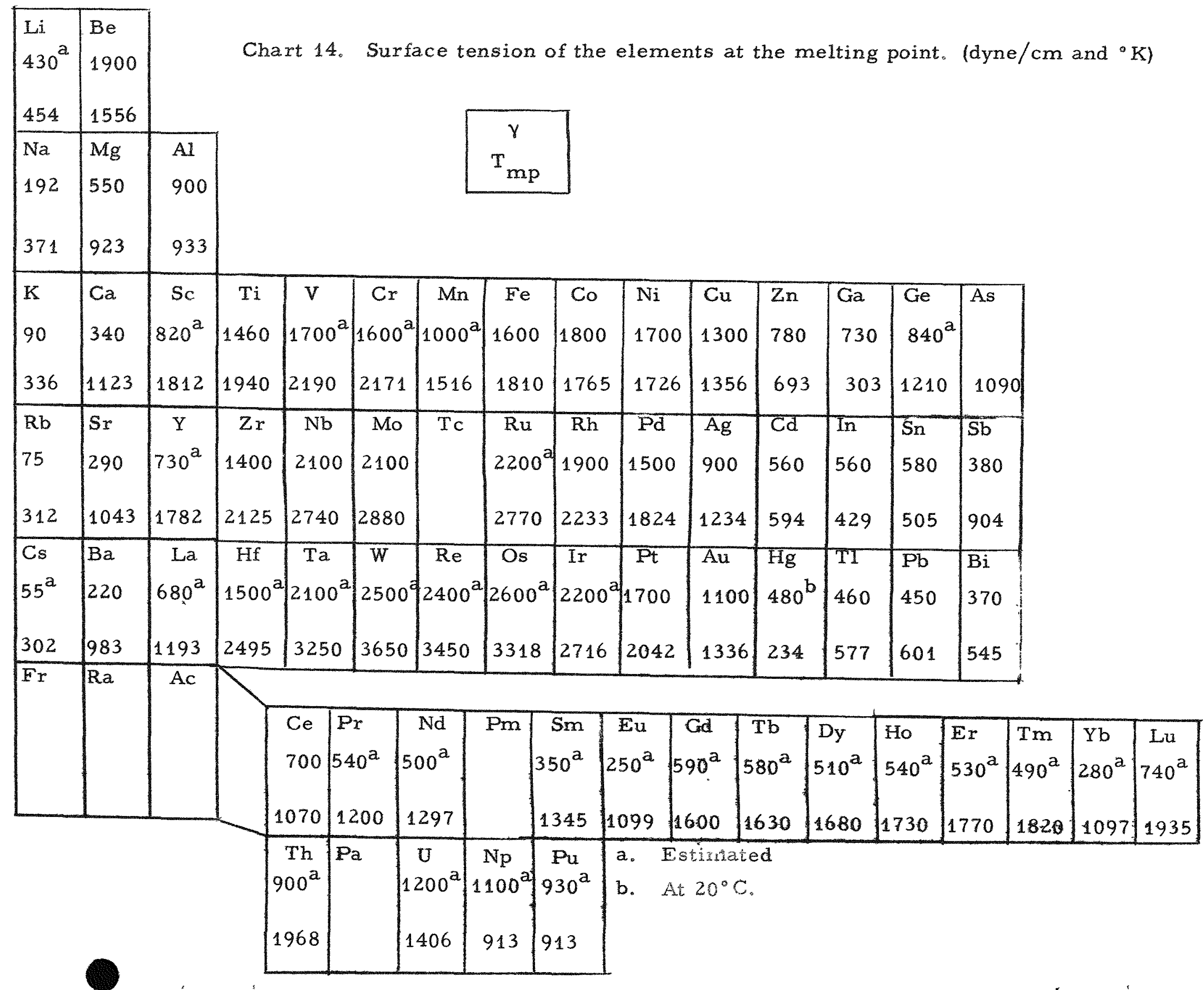




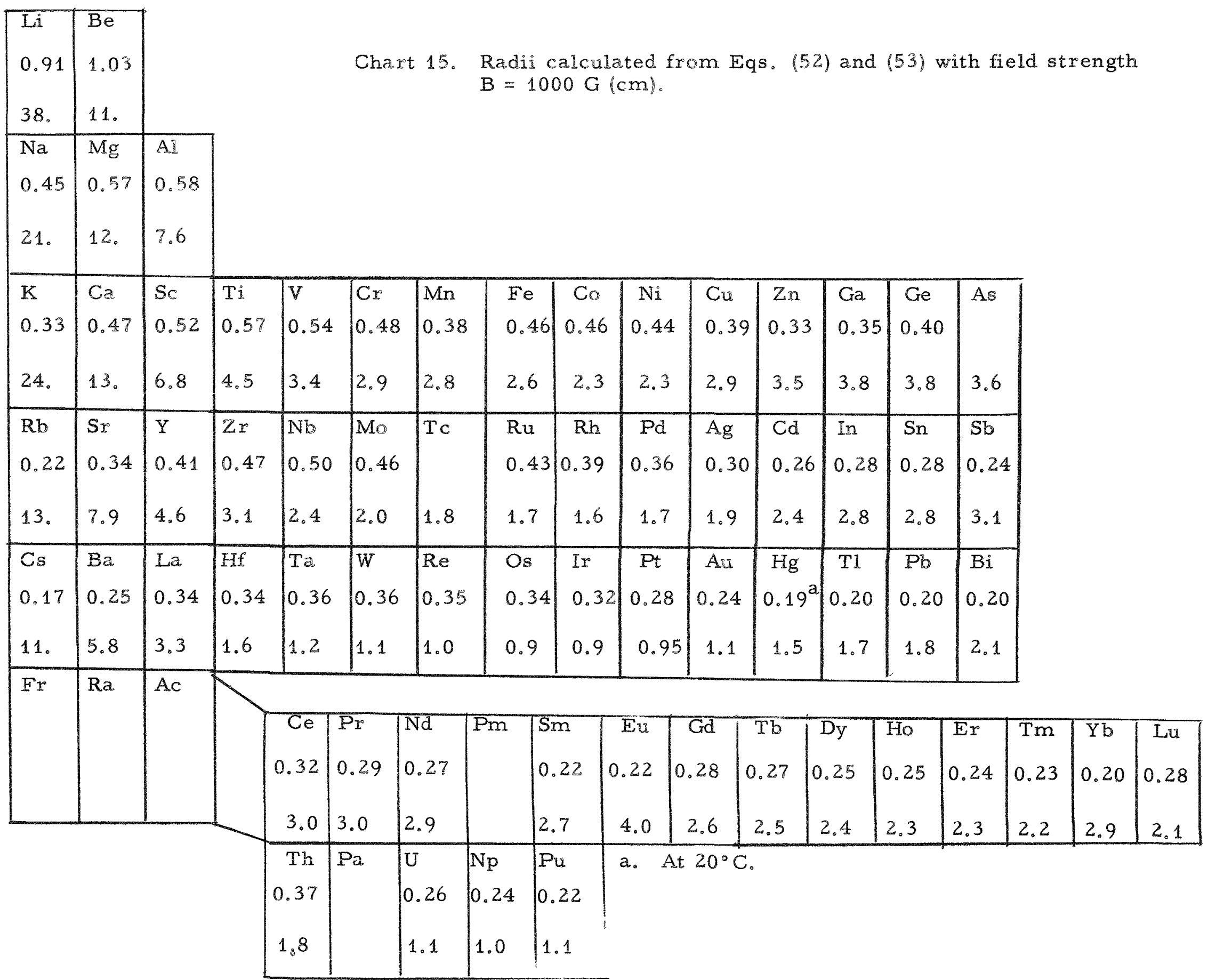


Even without considerations of dynamic stability, it can be concluded that levitation will probably not be widely applicable to the full-scale commercial production of pure metals. A possible exception which appears at present is the development of statically and dynamically stable levitation techniques for large plane metal sheets, long rods, or other systems designed to minimize the hydrostatic head of metal that needs to be balanced by the electromagnetic field.

\section{FUTURE DEVELOPMENTS}

A. Improved Induction Heatexs

In previous chapters it has been shown that separate control of both the frequency and magnitude of the sinusoidally alternating magnetic field is very desirable for laboratory-scale levitation work. Unfortunately, no commercial induction heater exists that can be either continuously or decade tuned over frequency range that spans even two orders of magnitude, such as $500 \mathrm{cps}$ to $50 \mathrm{kc} / \mathrm{sec}$. The uswal induction heating devices are (a) line frequency heaters for large metal pieces, (b) motor generators, which commonly operate in the 1 to $10 \mathrm{kc} / \mathrm{sec}$ range, or (c) electron-tube oscillators, which generally operate at fixed frequencies such as $100 \mathrm{kc} / \mathrm{sec}, 450 \mathrm{kc} / \mathrm{sec}$, or higher into the $r$ range. At best, the frequency tuning range for any of these devices is limited.

This situation is unfortunate, since there is an optimum frequency for the levitation of a given metal sample, depending not only on its size, shape, but also on its resistivity at the desired operating temperature. This point has been illustrated for solid-metal spheres of $1 \mathrm{~m} \mathrm{~cm}$ radius in Charts 6 and 12. If too high a frequency is used, simplicity, low cost, and power are sacrificed. The coil losses at high frequencies are very large, although Litz wire can occasionally be used to minimize them. 
In Chart 12, it is shown that the highest frequency needed to levitate a 1-cm-radius liquid sample of most metals is $60 \mathrm{kc} / \mathrm{sec}$. For many of the common metals, frequencies as $10 \mathrm{w}$ as $20 \mathrm{kc} / \mathrm{sec}$ are very satisfactory. Thus, there is no real advantage to levitating metals at $450 \mathrm{kc} / \mathrm{sec}$, as is done by the two commercial levitation devices, unless (a) small samples are used, or (b) high powers are desired. Jenkins, ${ }^{11,21}$ Weisberg, ${ }^{26}$ and others have commented on the fact that temperature control in vacuum levitation work is generally poor, with the result that the levitated metal samples have a tendency to "burn up." These problems can be reduced by operating at lower frequencies.

What is the ideal levitation device? From previous calculations, it has been shown that fields as high as several $\mathrm{kG}$ are quite desirable if they can be conveniently generated over toeasonable field volumes, although a device providing $500 \mathrm{G}$ over a $75 \mathrm{~cm}^{3}$ volume is probably more than adequate for most laboratory applications. This field can be generated through the use of (a) high current, low voltage, and few-turn air-corecoils, (b) lowcurrent, high-voltage, multiturn air-core coils, or perhaps (c) multiturn coils wound Qver magnetic cores. Ferrites or low-loss laminated-core materials such as Silectron ${ }^{37}$ can be used at frequencies as high as $50 \mathrm{kc} / \mathrm{sec}$ as transformers and perhaps to produce the field distribution directly. ${ }^{4}$ The frequency of this device would be adjustable in the range from at least $500 \mathrm{cps}$ to $50 \mathrm{kc} / \mathrm{sec}$, with a decade capacitor bank provided to tune the levitationcoil inductance.

In this frequency ronge, the use of motor generators with variablespeed control and/or frequency multipliers is most inconvenient. Fortun ately, recent advances in high-power solid-state active devices have made it possible to construct different and better induction heaters. Simpson 38 
has discussed the use of a transistor inverter as an induction heater. This is a switching device capable of converting dc to ac power of the order of at least several kilowatts. The resulting waveform is a square wave, the fundamental mode being the dominant one in induction heating and levitation. It is useful to quote Simpson on these points:

"Frequencies as high as $12 . \mathrm{kc}$ have been reported, and it is possible that higher frequencies could be achieved with improved transistors. Power ratings can be increased by utilizing transistors in series, enabling a higher input voltage to be used. Even with transistors available at present, an inverter of several kilowatts at $10 \mathrm{kc}$ and 300 to 500 volts is a practical possibility. Efficiencies of these inverters are very high (95 per cent), and the future potentialities of combining them with high-efficiency silicon diode dc power supplies are very great. The weight and cost would be low, and the variable frequency factor of transistor inverters gives them a great advantage over rotary equipment."

High-frequency power transistors have been further improved, and other efficient $s$ witching devices, such as the silicon-controlled rectifier (SCR) and the gate turn-off switch (GTO), have been discovered. The first two devices have definite limitations, the power transistor being inherently a. low-voltage device, and the SCR being difficult to turn off cnce it has been pulsed into conduction. The gate turn-off switch, the most recently discovered device, shows promise of considerable improvement over the other two. It can be turned off and on from a single gate terminal, operates at frequencies as high as $100 \mathrm{kc} / \mathrm{sec}$, needs only low-power pulses to turn it on or off, and can handle much higher load power for the same current 
rating since it is inherently a high-voltage device. Its main drawback at present is the fact that it is still a low-power device, the largest commercial one being rated at 7.5 amperes and 500 to 600 volts. Grafham ${ }^{39}$ gives further details.

The day when laboratory-scale induction heating will be revolutionized is not far off. Such devices will do much to simplify the application of induction heaters to levitation problems.

\section{B. Levitation of Dielectric Materials}

Levitation techniques have found their most useful applications in the research laboratory, where physicochemical studies of gas-metal reactions, vapor plating, sintering, vaporization phenomena, thermodynamics, and alloy preparation can be performed relatively easily on small levitated metal specimens. Analogous methods for levitating solic and liquid dielectrics in a cusped geometry similar to that used in metal levitation have not been found.

A method for levitating and heating solid dielectrics such as refractory oxides, nitrides, and carbides, other metal salts, and polymers is achieved by surrounding a light and unreactive metal sphere of high electrical conductivity by the dielectric material desired, and then levitating the composite sphere as outlined in the rest of the paper. This method involves the same temperature control problems as does the levitation of metals. If these problems can be met by suitable coil design, the use of high-thermalconductivity gases, or low frequencies, levitation techniques can be applied to physicochemical studies of these materials up to their melting, softening, or decomposition temperatures. Metal salt-gas reactions studied by this technique may have advantages over other methods. 
The most suitable metals for the core materials are those that are easiest to levitate, are unreactive, and have a high melting point. Beryllium meets these conditions, although low-melting-point metals such as aluminum, magnesium, sodium, lithium, and potassium may be used for specialized applications. A double dielectric coating can be used on these latter metals, one to render the metal inactive, and the other the one to be studied. A molten electrolyte may remain on the surface of the suspended sphere as a consequence of its surface tension and adhesion properties.

\section{VI, SUMMARY AND CONCLUSIONS}

1. Levitation melting techniques may not have widespread commercial application due both to the difficulties in scaling up the magnetic fields to levitate larger samples and to possible dynamic-instability problems. They have primary applicability in the research laboratory, where they can be used for a variety of metallurgical problems and physicochemical studies.

2. A possible exception to item 1 is the use of levitation heating or melting of large plane sheets of moderate thickness or long metal rods of moderate diameter. In this way, larger quantities can be melted without increasing the hydrostatic head of liquid, provided that these configurations are not dynamically unstable. One of the coil configurations developed by Foge1 ${ }^{40}$ may be useful for such applications.

3. More attention should be given to the improvement of commercial levitation equipment for the laboratory through the use of variable frequencies, solid-state circuits, and high-permeability magnetic-core materials with low power losses. The ideal levitation power supply would have a variable frequency control for the range of $500 \mathrm{cps}$ to 
50 or $100 \mathrm{kc} / \mathrm{sec}$, a decade capacitor bank, and the capability of producing a square or sine-wave magnetic field up to perhaps several kilogauss over a $75 \mathrm{~cm}^{3}$ volume. Gate turn-off switches seem most promising for this application.

4. An approach similar to the one taken in this paper can be made for zone refining techniques and other applications of induction heating.

5. For research into various problems connected with levitation--such as the shape of the liquid-metal specimen in the confining magnetic field, the minimum magnetic field needed for levitation, new coil geometries, and the use of magnetic cores to produce the magnetic-field distribution-the most suitable system is that of liquid sodium surrounded by a mineral ox silicone oil of nearly the same density. This minimizes the magnitude of the magnetic field and the frequency needed to study the phenomena.

6. To determine whether a given coil, field strength, and frequency will levitate a number of liquid metals, either liquid mercury or gallium surrounded by a coolant can be used to determine the characteristics of the system. If liquid mercury can be stably levitated, most other liquid metals can be also, provided that adequate temperature control is maintained. This substitutes easy-to-perform low-temperature experiments for high-temperature ones.

7. For vacuum metallurgy work in which a metal specimen is to be levitated for considerable time, levitation techniques can be used only with difficulty. Both the force on and the temperature of the levitated metal cannot be controlled with the power-control knob alone. For best results, separate control of both the frequency and the field intensity is needed. The power absorbed by the liquid metal is minimized by 
operating at the lowest frequency possible. Certain metals, because of their high density and electrical resistivity, are very difficult to levitation melt in a vacuum for considerable periods of time.

8. If the metallurgical studies can be performed in an inert-atmosphere environment, then the composition or pressure of a helium-argon mixture can be used to control the temperature of the liquid-metal specimen. Stable levitation of liquid metals can be achieved in this manner for considerable periods of time.

9. Levitation techniques can be applied to semiconductors and insulators at high temperatures, when their electrical resistivities are low.

10. A solid dielectric material can be levitated and heated under controlled conditions if it surrounds a light unreactive metal of high electrical conductivity and melting point. This technique permits physicochemical studies of dielectric materials similar to those performed for levitated metals.

11. There is a greater force on a levitated metal sample if the axis of a symmetrical levitation coil is horizontal instead of vertical. The power is greater also.

12. High-frequency magnetic-core materials may have possible application to the production of magnetic-field distributions in levitation work.

13. The criteria for the dynamic stability of a levitated liquid-metal sample have yet to be explicitly stated.

14. Techniques used to stabilize and confine gaseous plasmas may have applications to metal levitation. 


\section{APPENDIX}

This appendix describes in detail the derivation of the fundamental levitation equations. Consider a homogeneous body of arbitrary size and shape, constant permittivity $\epsilon$, permeability $\mu$, and conductivity $\sigma$, surrounded by a nonconducting medium characterized by the free-space permittivity $\epsilon_{0}$ and permeability $\mu_{0}$. The fundamental laws of electromagnetic theory (in rationalized mks units), which apply to both the body and the surrounding medium, are given by Maxwell's equations,

$$
\begin{aligned}
& \nabla \times \frac{\mathrm{E}}{m}=-\frac{\partial \mathrm{B}}{\partial \mathrm{t}} \\
& \nabla \times \underset{m}{\mathrm{H}}=\mathrm{J}+\frac{\partial \mathrm{D}}{\partial t} \\
& \nabla \underset{m}{\mathrm{~B}}=0 \\
& \nabla \cdot \underset{m}{\mathrm{D}}=\rho^{\prime},
\end{aligned}
$$

the assumed linear constiuctive relationships between the various vector quantities within the homogeneous body,

$$
\begin{aligned}
& \mathrm{D}=\epsilon \underset{\infty}{\mathrm{E}} \\
& \mathrm{B}=\mu \mathrm{H} \\
& \mathrm{J}=\sigma \mathrm{m},
\end{aligned}
$$

and the rigorously linear conet" "ve relationships between the various vector quantities in the surrounding nonconducting medium,

$$
\begin{aligned}
& D=\epsilon_{0} \underset{m}{\mathrm{E}} \\
& \mathrm{B}=\mu_{0} \underset{\infty}{\mathrm{H}} .
\end{aligned}
$$

The electric and magnetic fields are now assumed to be steady-state sinusoidally alternating fields. In spherical coordinates, the fields are of the form

$$
V=\vec{V}^{\prime}(r, \theta, \phi) \cos [\omega t-a(r, \theta, \phi)]
$$


where it is noticed that both the peak amplitude $\vec{V}^{\prime}$ and phase $a$ of the vector field may vary from point to point. This somewhat awkward representation for the field can be simplified by the use of the complex exponential $e^{j(\omega t-a)}$, whose time dexivative is

$$
\frac{\partial}{\partial t} e^{j(\omega t-a)}=j \omega e^{j(\omega t-a)},
$$

where $\quad j=(-1)^{1 / 2}$

$\omega=$ radian frequency

$t=$ time

Thus,

$$
V=\operatorname{Re}\left[\vec{V}^{\imath}(r, \theta, \phi) e^{j[\omega t-a(r, \theta, \phi)]}\right]=\operatorname{Re}\left[\vec{V}^{\prime}(r, \theta, \phi) e^{-a(r, \theta, \phi)} e^{j \omega t}\right] .
$$

For simplicity of terminology, the $\operatorname{Re}$ symbol is omitted and $V$ is simplified to

$$
V=\vec{V}(r, \theta, \phi) e^{j \omega t}
$$

where

$$
\vec{V}(r, \theta, \phi)=\vec{V}^{\prime}(r, \theta, \phi) e^{-a(r, \theta, \phi)} \text {. }
$$

Similarly,

$$
\rho^{\prime}=\operatorname{Re}\left[\rho^{\prime}(r, \theta, \phi) \mathrm{e}^{-a\left(r_{0}, \theta, \phi\right)} \mathrm{e}^{j \omega t}\right]=\rho(r, \theta, \phi) \mathrm{e}^{j \omega t} .
$$

For further discussions of these points, Adler, Chu, and Fano ${ }^{41}$ or Moon and Spencer ${ }^{42}$ should be consulted.

Equations (A-1) simplify to

$$
\begin{aligned}
& \nabla \times \vec{E}=-j \omega \mu \vec{H} \\
& \nabla \times \vec{H}=\vec{J}+j \omega \in \vec{E} \\
& \nabla \cdot \vec{B}=0 \\
& \nabla \cdot \vec{D}=\rho,
\end{aligned}
$$

as do the constitutive relationships for the homogeneous body [Eq. (A-2)] , 


$$
\begin{aligned}
& \vec{D}=\epsilon \overrightarrow{\mathrm{E}} \\
& \vec{B}=\mu \overrightarrow{\mathrm{H}} \\
& \vec{J}=\sigma \overrightarrow{\mathrm{E}}
\end{aligned}
$$

and the constitutive relationships for the surrounding medium [Eq. $(A-3)]$,

$$
\begin{aligned}
& \overrightarrow{\mathrm{D}}=\epsilon_{0} \overrightarrow{\mathrm{E}} \\
& \overrightarrow{\mathrm{B}}=\mu_{0} \overrightarrow{\mathrm{H}} .
\end{aligned}
$$

If it is assumed that no free charges exist either inside or outside the body, $\rho=0$ and the above equations reduce to the vector Helmholtz equations for a homogeneous body,

$$
\begin{aligned}
& \nabla^{2} \overrightarrow{\mathbf{E}}+\left(\omega^{2} \epsilon \mu-j \omega \mu \sigma\right) \overrightarrow{\mathbf{E}}=0 \\
& \nabla^{2} \overrightarrow{\mathrm{B}}+\left(\omega^{2} \epsilon \mu-j \omega \mu \sigma\right) \overrightarrow{\mathrm{B}}=0 \\
& \nabla^{2} \overrightarrow{\mathrm{D}}+\left(\omega^{2} \epsilon \mu-j \omega \mu \sigma\right) \overrightarrow{\mathrm{D}}=0 \\
& \nabla^{2} \overrightarrow{\mathrm{H}}+\left(\omega^{2} \epsilon \mu-j \omega \mu \sigma\right) \overrightarrow{\mathrm{H}}=0 \\
& \nabla^{2} \overrightarrow{\mathrm{J}}+\left(\omega^{2} \epsilon \mu^{\prime}-j \omega \mu \sigma\right) \overrightarrow{\mathrm{J}}=0
\end{aligned}
$$

and for the surrounding nonconducting medium,

$$
\begin{aligned}
& \nabla^{2} \vec{E}+\omega^{2} \epsilon_{0} \mu_{0} \vec{E}=0 \\
& \nabla^{2} \vec{B}+\omega^{2} \epsilon_{0} \mu_{0} \vec{B}=0 \\
& \nabla^{2} \vec{D}+\omega^{2} \epsilon_{0} \mu_{0} \vec{D}=0 \\
& \nabla^{2} \vec{H}+\omega^{2} \epsilon_{0} \mu_{0} \vec{H}=0,
\end{aligned}
$$

where advantage has been taken of the vector identity.

$$
\nabla \times \nabla \times \vec{V}=\nabla(\nabla \cdot \vec{V})-\nabla^{2} \vec{V}
$$

In order to solve these equations, it is useful to define a vector potential, A,

$$
\begin{aligned}
& B=\nabla \times \underset{\infty}{B}= \\
& E=-\frac{\partial A}{\partial t}
\end{aligned}
$$


where it is assumed that $\nabla V=0$. A will also vary sinusoidally, as in

$$
A=\vec{A} e^{j \omega t} \text {, }
$$

so that

$$
\vec{E}=-j \omega \vec{A}
$$

and

$$
\nabla^{2} \vec{A}+\left(\omega^{2} \epsilon \mu-j \omega \mu \sigma\right) \vec{A}=0
$$

For metals, $\sigma$ is so high that for any ordinary frequency

$$
j \omega \mu \sigma>>\omega^{2} \mu \epsilon \text {. }
$$

Thus, Eq. $(A-17)$ reduces to

$$
\nabla^{2} \overrightarrow{\mathrm{A}}=j \omega \mu \sigma \overrightarrow{\mathrm{A}},
$$

which is the basic relationship used to derive the levitation equations.

Consider now a metal sphere of $\sigma, \mu$, and $R$ placed in a sinusoidal alternating $\mathrm{z}$-directed magnetic field,

or

$$
\underset{m}{B}=B e^{j \omega t} \vec{e}_{z}, \quad \text { cylindrical coordinates }
$$

$$
\underset{m}{B}=\left[B \cos \theta \vec{e}_{r}-B \sin \theta \vec{e}_{\theta}\right] e^{j \omega t} \text { spherical coordinates. }
$$

From Eq. $(A-14)$, the vector potential for this $z$-directed magnetic field is

$$
A_{m}=A_{\phi}(r, \theta) e^{j \omega t} \vec{e}_{\phi}=\frac{1}{2} B r \sin \theta e^{j \omega t} \vec{e}_{\phi} .
$$

The fact that the vector potential has a $\phi$ component only simplifies the mathematics considerably and is the main reason for introducing it rather then solving Eq. $(A-11)$ for $B$ directly. The left-hand side of $(A-19)$ now becomes

$$
\nabla^{2} \overrightarrow{\mathrm{A}}_{\phi}=\overrightarrow{\mathrm{e}}_{\phi} \nabla^{2} \mathrm{~A}_{\phi}+\mathrm{A}_{\phi} \nabla^{2} \overrightarrow{\mathrm{e}}_{\phi}=\left[\nabla^{2} \mathrm{~A}_{\phi}-\frac{\mathrm{A}_{\phi}}{\mathrm{r}^{2} \sin ^{2} \theta}\right] \overrightarrow{\mathrm{e}}_{\phi},
$$


where $\nabla \vec{e}_{\phi}^{2}=-\frac{1}{r^{2} \sin ^{2} \theta} \vec{e}_{\phi}$. Therefore

$$
\nabla^{2} A_{\phi}-\frac{A_{\phi}}{r^{2} \sin ^{2} \theta}-j \omega \mu \sigma A_{\phi}=0
$$

or, by expansion of $\nabla^{2} \mathrm{~A}_{\phi}$,

$$
\frac{1}{r^{2}} \frac{\partial}{\partial r}\left(r^{2} \frac{\partial A_{\phi}}{\partial r}\right)+\frac{1}{r^{2} \sin \theta} \quad \frac{\partial}{\partial \theta}\left(\sin \theta \frac{\partial A_{\phi}}{\partial \theta}\right)-\frac{A_{\phi}}{r^{2} \sin ^{2} \theta}-j \omega \mu \sigma A_{\phi}=0 .
$$

If the substitution $u=\cos \theta$ is made, Eq. $(A-24)$ becomes

$$
\frac{1}{r^{2}} \frac{\partial}{\partial r}\left(r^{2} \frac{\partial A_{\phi}}{\partial r}\right)+\frac{\left(1-u^{2}\right)^{1 / 2}}{r^{2}} \frac{\left.\partial^{2}\left[1-u^{2}\right)^{1 / 2} A_{\phi}\right]}{\partial u^{2}}-j \omega \mu \sigma A_{\phi}=0 .
$$

The solution to Eq. $(A-25)$ is of the form,

$$
A_{\phi}=r^{-1 / 2} R \Theta
$$

Substituting into the equation, multiplying by $\mathrm{r}^{2}$, and dividing by $\mathrm{A}_{\phi}$, gives

$$
\frac{r^{2}}{R} \frac{\partial^{2} R}{\partial r^{2}}+\frac{x}{R} \frac{\partial R}{\partial r}-\mu j \omega \mu \sigma r^{2}=-\frac{\left(1-u^{2}\right)^{1 / 2}}{\Theta} \frac{\partial^{2}\left[\left(1-u^{2}\right)^{1 / 2} \Theta\right]}{\partial u^{2}} .
$$

By setting both sides equal to $n(n+1)$ and expanding the derivatives, the equations become

$$
\begin{aligned}
& \left(1-u^{2}\right) \frac{d^{2} \Theta_{n}}{d u^{2}}-2 u \frac{d \Theta_{n}}{d u}-\frac{\Theta_{n}}{1-u^{2}}+n(n+1) \Theta_{n}=0 \\
& \frac{d^{2} R_{n}}{d x^{2}}+\frac{1}{r} \frac{d R_{n}}{d r}-\left[j \omega \mu \sigma+\frac{n(n+1)+\frac{1}{4}}{r^{2}}\right] R_{n}=0 .
\end{aligned}
$$


Equation $(A-28)$ is the differential equation for associated Legendre functions $^{17}$ whereas Eq. (A-29) is the differential equation for modified Bessel functions. 17 The final solution for the interior region of the metal sphere is $A_{\phi}=r^{-1 / 2}\left[A_{n} P_{n}^{1}(u)+B_{n} Q_{n}^{1}(u)\right]\left[C_{n} I_{n+1 / 2}(j \omega \mu \sigma)^{1 / 2} r+D_{n} K_{n+1 / 2}(j \omega \mu \sigma)^{1 / 2} r\right]$.

If $n$ is an integer, $I_{-(n+1 / 2)}$ can be used in place of $K_{n+1 / 2}$.

In the region outside the metal sphere where the conductivity of the medium is zero, the vector equation analogous to Eq. (A-19) is

$$
\nabla^{2} \vec{A}+\omega^{2} \epsilon_{0} \mu_{0} \vec{A}=0 \text {. }
$$

which, when expanded and the substitution $u=\cos \theta$ made, becomes identical to Eq. $(A-25)$ with the exception that $-j \omega \mu \sigma$ is replaced by $\omega^{2} \epsilon_{0} \mu_{0}$. Consequently, the solution is the same form as Eq. (A-26),

$$
A_{\phi}^{\prime}=r^{-1 / 2} R^{\prime} \Theta
$$

where Eq. (A-28) again applies. With the substitution of $\omega^{2} \epsilon_{0} \mu_{0}$ for $-j \omega \mu \sigma$ into Eq. (A-29), it becomes the differential equation for Bessel functions. ${ }^{17}$ The final solution for the nonconducting region exterior to the metal sphere is

$$
\begin{aligned}
& A_{\phi}^{\prime}=r^{-1 / 2}\left[A_{n}^{\prime} P_{n}^{1}(u)+B_{n}^{\prime} Q_{n}^{1}(u)\right]\left[C_{n}^{\prime \prime} J_{n+1 / 2}\left(\omega^{2} \epsilon_{0} \mu_{0}\right)^{1 / 2} r\right. \\
& \left.+D_{n}^{n J}-(n+1 / 2)^{\left(\omega^{2} \epsilon_{0} \mu_{0}\right)^{1 / 2}} \mathrm{r}\right]
\end{aligned}
$$

At ordinary frequencies, $\left(\omega^{2}{ }_{0} \mu_{0}\right)^{1 / 2}$ is a very small number, i. e. , it is equal to only 0.0033 meter $^{-1}$ when $\omega=10^{6} \mathrm{rad} / \mathrm{sec}$. Consequently, for the nonconducting exterior region, it is a very good approximation to remove the

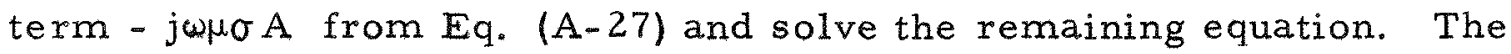


solution now takes the form

$$
A_{\phi}^{\prime}=R^{\prime} \Theta
$$

Again, the value of $\Theta_{n}$ can be obtained from Eq. (A-28), which remains unchanged. Equation (A-29) becomes

$$
\frac{d}{d r}\left(r^{2} \frac{d R_{n}^{\prime}}{d r}\right)-n(n+1) R_{n}^{\prime}=0
$$

which has a solution

$$
R_{n}^{\prime}=C_{n}^{\prime} r^{n}+D_{n}^{\prime} r^{-n-1}
$$

Thus, the simplified solution for the nonconducting region is

$$
A_{\phi}^{\prime}=\left[A_{n}^{1} P_{n}^{1}(\cos \theta)+B_{n}^{\prime} Q_{n}^{1}(\cos \theta)\right]\left[C_{n}^{\prime} r^{n}+D_{n}^{\prime} r^{-n-1}\right]
$$

where $u=\cos \theta$. By use of the definition of $P_{1}^{1}(\cos \theta)$,

$$
\mathrm{P}_{1}^{1}(\cos \theta)=\left(1-\cos ^{2} \theta\right)^{1 / 2}=\sin \theta
$$

and comparison of Eqs. $(A-22)$ and $(A-37)$, it is seen that, for the present system, $\mathrm{n}=1$ and $\mathrm{B}_{1}^{1}=0$.

The solutions $(A-30)$ and $(A-37)$ are subject to four boundary conditions, which determine the values of the unknown constants:

1. Outside the metal sphere, the vector potential due to the eddy currents must vanish at infinity

$$
A_{\phi}^{\prime}=\frac{1}{2} B r \sin \theta \quad \text { at } r=\infty .
$$

2. Inside the metal sphere, the eddy-current-vector potential must remain finite at the center

$$
A_{\phi}=\text { finite at } r=0 \text {. }
$$


3. Across the phase boundary, the vector potential must be continuous

$$
\vec{A}=\vec{A}^{\prime} \quad \text { at } r=R \text {. }
$$

4. Across the phase boundary, the tangential component of $\mathrm{H}$, the magnetic-field intensity, must be continuous

$$
\vec{n} \times \frac{\nabla \times \vec{A}}{\mu}=\vec{n} \times \frac{\nabla \times \vec{A}}{\mu_{0}} \quad \text { at } r=R \text {. }
$$

From the first boundary condition,

$$
A_{1}^{\prime} C_{1}^{\prime}=\frac{1}{2} B
$$

and from the second,

$$
\mathrm{D}_{1}=0
$$

Equations $(A-30)$ and $(A-37)$ simplify to

$$
\begin{aligned}
& A_{\phi}=\frac{1}{2} B C r^{-1 / 2} \sin \theta I_{3 / 2}(j \omega \mu \sigma)^{1 / 2} r \\
& A_{\phi}^{\prime}=\frac{1}{2} B r \sin \theta+D_{1}^{\prime} r^{-2} \sin \theta
\end{aligned}
$$

where $C=C_{1} / C_{1}$. By comparison of Eqs. $(A-22)$ and $(A-46)$, it is seen that Eq. (A-46) can be written,

$$
\begin{aligned}
A_{\phi}^{\prime} & =\left\{\begin{array}{l}
\text { vector potential due to the } \\
z \text { mirected magnetic field }
\end{array}\right\}+\left\{\begin{array}{l}
\text { vector potential due to } \\
\text { the eddy currents }
\end{array}\right\} \\
& =A_{\phi}+A_{\phi}^{\prime \prime},
\end{aligned}
$$

where

$$
A_{\phi}^{\prime \prime}=D_{1}^{\prime} r^{-2} \sin \theta
$$

which is a fundamental result for levitation theory.

From page 270 or Smythe, 17 the vector potential for a very small circular loop of radius $R$ is given by

$$
\vec{A}=\mu_{0} \frac{\vec{M} \times \vec{r}}{4 \pi r^{3}}=\mu_{0} \frac{M \sin \theta}{4 \pi r^{2}} \vec{e}_{\phi},
$$


where $M$, the magnetic moment of the loop, is

$$
M=\pi R^{2} I \text {. }
$$

Thus, the eddy-current field of a metal sphere is like that of a small magnetic dipole loop of radius $R$ carrying a current $I e^{j \omega t}$, where

$$
M=\frac{4 \pi D_{1}^{\prime}}{\mu_{0}} .
$$

All that is necessary now is to determine the constant $D_{1}^{\prime}$ from the remaining two boundary conditions (A-41) and (A-42),

$$
\begin{aligned}
& A_{\phi}=A_{\phi}^{\prime} \\
& \mu_{0} \frac{\partial}{\partial r}\left(r \sin \theta A_{\phi}^{\vdots}\right)=\mu \frac{\partial}{\partial r}\left(r \sin \theta A_{\phi}^{\prime}\right),
\end{aligned}
$$

or

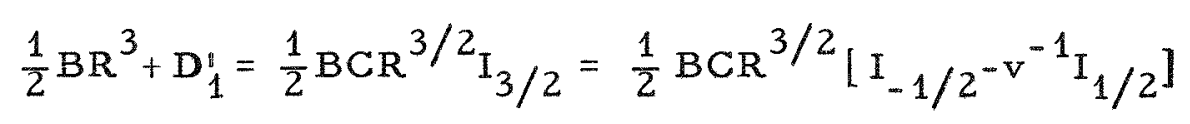

$$
\begin{aligned}
& \mathrm{BR}^{3}-\mathrm{D}_{1}^{\prime}=\frac{1}{2} \mathrm{BCR}^{3 / 2} \frac{\mu_{0}}{\mu}\left[\frac{1}{2} \mathrm{I}_{3 / 2}+\mathrm{vI}_{3 / 2}^{\prime}\right]=\frac{1}{2} \mathrm{BCR}^{3 / 2} \\
& \left\{\frac{\mu_{0}}{\mu}\left[\left(v+v^{-1}\right) I_{1 / 2^{-I}-1 / 2}\right]\right\}
\end{aligned}
$$

where $I_{3 / 2}, I_{1} / 2, I_{-1 / 2}$, and $v$ are Smythe's shorthand notations

$$
\begin{gathered}
\mathrm{v}=(j \omega \mu \sigma)^{1 / 2} \mathrm{R} \\
I_{3 / 2}=I_{3 / 2}(\mathrm{v})=\left(\frac{2}{\pi \mathrm{v}}\right)^{1 / 2}\left[\cosh \mathrm{v}-\frac{1}{\mathrm{v}} \sinh \mathrm{v}\right] \\
I_{1 / 2}=I_{1 / 2}(\mathrm{v})=\left(\frac{2}{\pi v}\right)^{1 / 2} \sinh \mathrm{v} \\
I_{-1 / 2}=I_{-1 / 2}(v)=\left(\frac{2}{\pi v}\right)^{1 / 2} \cosh \mathrm{v} .
\end{gathered}
$$


The solutions for $C$ and $D_{1}^{\prime}$ are

$$
\begin{aligned}
& C=\frac{3 R^{3 / 2} \mu v}{\left(\mu-\mu_{0}\right) v I_{-1 / 2}+\left[\mu_{0}\left(1+v^{2}\right)-\mu\right] I_{1 / 2}} \\
& D_{1}^{\prime}=\frac{\left(2 \mu+\mu_{0}\right) v I_{-1 / 2}-\left[\mu_{0}\left(1+v^{2}\right)+2 \mu\right] I_{1 / 2}}{\left(\mu-\mu_{0}\right) v I_{-1 / 2}+\left[\mu_{0}\left(1+v^{2}\right)-\mu\right] I_{1 / 2}} \frac{1}{2} B R^{3} .
\end{aligned}
$$

In order to simplify $C$ and $D_{1}^{\prime}$ in Eqs. (A-57), the following hyperbolic, trigonometric, and modified Bessel function identities are needed:

$$
\begin{aligned}
& v=(j \omega \mu \sigma)^{1 / 2} R=(1+j) x \\
& v^{*}=(-j \omega \mu \sigma)^{1 / 2} R=(1-j) x \\
& \mathrm{x}=\left(\frac{\omega \mu \sigma}{2}\right)^{1 / 2} \mathrm{R} \text {; } \\
& \sinh (1 \pm j) x=\sinh x \cos x \pm j \cosh x \sin x \\
& \cosh (1 \pm j) x=\cosh x \cos x \pm j \sinh x \sin x \\
& \cosh ^{2} x-\sinh ^{2} x=1 \\
& \sin ^{2} x+\cos ^{2} x=1 \\
& \sin 2 x=2 \sin x \cos x \\
& \sinh 2 x=2 \sinh x \cosh x \\
& \sinh ^{2} x+\sin ^{2} x=\frac{1}{2}(\cosh 2 x-\cos 2 x) ; \\
& \left.I_{1 / 2} I_{-1 / 2}=I_{1 / 2}(v) I_{-1 / 2^{(v)}} v^{* *}\right)=\frac{1}{\pi x 2^{1 / 2}}(\sinh 2 x+j \sin 2 x) \\
& I_{1 / 2}^{*} I_{-1 / 2}=\frac{1}{\pi \times 2^{1 / 2}}(\sinh 2 x-j \sin 2 x)
\end{aligned}
$$

(A-60 continued) 


$$
\begin{aligned}
& I_{1 / 2} I_{1 / 2}^{* *}=\frac{2^{1 / 2}}{\pi x}\left(\sinh ^{2} x+\sin ^{2} x\right) \\
& I_{-1 / 2} I_{-1 / 2}^{*}=\frac{2^{1 / 2}}{\pi x}\left(\cosh ^{2} x-\sin ^{2} x\right)
\end{aligned}
$$

$$
\begin{aligned}
\int_{0}^{\mathrm{R}} r \mathrm{I}_{3 / 2}(\ddot{v}) I_{3 / 2}\left(\widetilde{v}^{* *}\right) d r & =\frac{1}{2 \omega \mu \sigma}\left[v^{* *} I_{-1 / 2}^{*} I_{1 / 2}+v I_{-1 / 2} I_{1 / 2}^{*}-2 I_{1 / 2} I_{1 / 2}^{*}\right] \\
& =\frac{x(\sinh 2 x+\sin 2 x)-2\left(\sinh ^{2} x+\sin ^{2} x\right)}{(\omega \mu \sigma)^{3 / 2} \pi R}(A-61)
\end{aligned}
$$

where the asterisk ${ }^{*}$ denotes the complex conjugate and $\widetilde{v}=(j \omega \mu \sigma)^{1 / 2}{ }_{x}$. From the definition of the dimensionless parameter $x$ given in Eq. (A-58), it is evident that $\mathrm{x}$ is the ratio of the radius of the metal sphere to the skin depth

$$
x=\frac{R}{\delta}
$$

This dimensionless group is of fundamental importance not only to levitation melting but to the entire area of induction heating.

For nonferrous metals, $\mu=\mu_{0}$, and Eq. $(\mathrm{A}-57)$ becomes

$$
\begin{aligned}
-\frac{D_{1}^{\prime}}{\frac{1}{2} B R^{3}} & =1-3 \frac{v I-1 / 2-I_{1 / 2}}{v^{2} I_{1 / 2}}=1-3 \frac{v \cosh v-\sinh v}{v^{2} \sinh v} \\
& =1-\frac{3}{4 x} \frac{\sinh 2 x-\sin 2 x}{\sinh ^{2} x+\sin ^{2} x}=G(x) .
\end{aligned}
$$

For ferrous metals, $\mu>>\mu_{0}$ and

$$
\frac{D_{1}^{1}}{\frac{1}{2} B R^{3}}=2-\frac{3 \mu_{0} v^{2} I_{1 / 2}}{\mu v I_{-1 / 2}+\left[\mu_{0} v^{2}-\mu\right] I_{1 / 2}} \text {. }
$$


If $\mu>>\mu_{0} v^{2}$,

$$
\begin{aligned}
\frac{D_{1}^{\prime}}{\frac{1}{2} B R^{3}} & \approx 2-\frac{3 \mu_{0}}{\mu} \frac{v^{2} I_{1 / 2}}{v I_{-1 / 2}-I_{1 / 2}}=2-\frac{3 \mu_{0}}{\mu} \frac{\sinh ^{2} x+\sin ^{2} x}{\sinh 2 x-\sin 2 x} \\
& \approx 2 .
\end{aligned}
$$

The calculation of the force on the metal sphere follows the plan first suggested by R. C. Mason ${ }^{19}$ and also the derivation for an analogous problem, a dielectric sphere in an inhomogenous field, given by Abraham and Becker. ${ }^{43}$ The simplification applied is stated in Sec. II.B in the main body of the paper. The force is given by the simple formula

$$
\vec{F}=(\vec{M} \cdot \nabla) \vec{B} \text {. }
$$

By substitution of Eq. (A-62), this equation becomes, for nonferrous metals,

$$
\vec{F}=-\frac{2 \pi R^{3}}{\mu_{0}} G(x)(\vec{B} \cdot \nabla) \vec{B},
$$

where it is noted that the vectors $\vec{M}=M \vec{n}$ and $\vec{B}$ point in the same direction. For ferrous metals, Eq. (A-64) substituted into Eq. (A-65) leads to

$$
\overrightarrow{\mathrm{F}}=\frac{4 \pi \mathrm{R}^{3}}{\mu_{0}}(\overrightarrow{\mathrm{B}} \cdot \nabla) \overrightarrow{\mathrm{B}}
$$

Since the term $(\vec{B} \cdot \nabla) \vec{B}$ is negative and since

$$
\begin{aligned}
& \vec{F}=+(\text { repulsive force) } \\
& \vec{F}=-(\text { attractive force) }
\end{aligned}
$$

the forces in Eqs. (A-66) and (A-67) are repulsive and attractive respectively for nonferrous and ferrous spheres located near a loop of wire carrying an alternating current of low frequency. Equation (A-67) also indicates that, for low frequencies, the attractive force between the ferrous-metal sphere and the current loop is independent of the parameter $x$, which is a function of the frequency and the metal radius, conductivity, and permeability. 
Since a repulsive force is needed for levitation, calculations need be made for nonpermeable materials only. These calculations obviously also hold for permeable metals above their Curie points.

The power absorbed by the metal sphere is given by the formulas

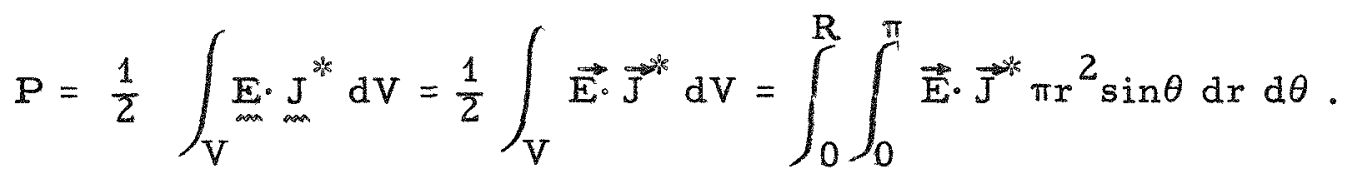

From Eqs. (A-9) and (A-16),

$$
P=\pi \omega^{2} \int_{0}^{R} \int_{0}^{\pi} A_{\phi} A_{\phi}^{*} r^{2} \sin \theta d r d \theta
$$

and from Eq. $(A-45)$

$$
\begin{aligned}
P & =\frac{\pi \omega^{2} \sigma B^{2}}{4} C C^{*} \int_{0}^{R} \int_{0}^{\pi} I_{3 / 2} I_{3 / 2^{*} \sin ^{3} \theta d r d \theta}^{R} \\
& =\frac{\pi \omega^{2} \sigma B^{2}}{3} C C^{*} \int_{0}^{R} I_{3 / 2} I_{3 / 2}^{*} r d r
\end{aligned}
$$

The value of the product of $C$ and its complex conjugate $C^{*}$ is needed to calculate the power absorbed by the sphere

$$
C C^{*}=\frac{9 R^{3} \pi}{x 2^{3 / 2}\left(\sinh ^{2} x+\sin ^{2} x\right)}=\frac{9 R^{3}}{v v^{*} I_{1 / 2} I^{*} 1 / 2}
$$

By substitution into Eq. $(A-70)$ the values of the integral and the product $\mathrm{CC}^{* \%}$ given in Eqs. $(A-61)$ and $(A-71)$, respectively, the final form for the power absorbed by the metal sphere is obtained

$$
P=\frac{3 \pi R B^{2}}{\sigma \mu_{0}^{2}}\left[\frac{x(\sinh 2 x+\sin 2 x)}{2\left(\sinh ^{2} x+\sin ^{2} x\right)}-1\right]=\frac{3 \pi R B^{2}}{\sigma \mu_{0}^{2}} H(x)
$$


where

$$
H(x)=\frac{x(\sinh 2 x+\sin 2 x)}{2\left(\sinh ^{2} x+\sin ^{2} x\right)}-1=\frac{x(\sinh 2 x+\sin 2 x)}{\cosh 2 x-\cos 2 x}-1
$$

which is again identical to the formula for $H(x)$ given by Okress et al. 19 Equation $(\mathrm{A}-72)$ can be rewritten, becoming

$$
P=\frac{3 \pi R}{\sigma \mu_{0}^{2}}(\vec{B} \cdot \vec{B}) H(x)
$$

To summarize, the key relationships of levitation theory for nonferrous metals are given by the equations describing the force on the sphere, the power absorbed by it, and the magnitude of the dimensionless group $\mathrm{x}$ :

$$
\begin{aligned}
& \vec{F}=-\frac{2 \pi R^{3}}{\mu_{0}}(\vec{B} \cdot \nabla) \vec{B} G(x) \\
& P=\frac{3 \pi R}{\sigma \mu_{0}^{2}}(\vec{B} \cdot \vec{B}) H(x) \\
& x=\left(\frac{1}{2} \omega \mu \sigma\right)^{1 / 2} R .
\end{aligned}
$$

Equations $(A-66)$ and $(A-73)$, the fundamental equations of levitation, show that the properties of the levitated metal sphere have been separated from the spatial characteristics of the applied alternating magnetic field. Since the frequency has also been separated, the terms $\vec{B} \cdot \vec{B}$ and $(\vec{B} \cdot \nabla) \vec{B}$ can be obtained from simple magnetostatic calculations. It must be emphasized that Eqs. (A-66) and (A-73) are approximations, being most valid for metal spheres of small size. 


\section{NOTATION}
a
Y
$\delta$
$€$
0
$\theta$
$\Theta$
$x_{1} * x_{2}$
$\mu$
$\mu_{0}$
$v$
$p$
$P_{0}$
$\sigma$
$\tau$
$\phi$
$\omega$
$\nabla$

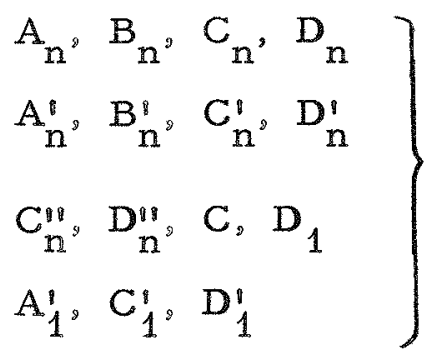
A
$A_{\phi}, A_{\phi}^{\prime}, A_{\phi}^{\prime \prime \prime}$
$\mathrm{B}$
D
Phase
Surface tension
Skin depth
Permittivity
Permittivity of free space
Coordinate direction
Specified function of $\theta$
Functions dependent on spatial characteristics
of magnetic field
Permeability
Permeability of free space
Frequency
Density; reduced radial distance
Density of the external dielectric medium
Electrical conductivity
Electrical resistivity
Coordinate direction
Radian frequency
Del operator
Integration constants
Vector potential
Vector potential in $\phi$ direction
Magnetic induction
Electric displacement 
d

E

E, $E_{I}, E_{I I}$

$\overrightarrow{\mathrm{e}}_{\mathrm{r}}, \overrightarrow{\mathrm{e}}_{z}, \overrightarrow{\mathrm{e}}_{\phi}, \overrightarrow{\mathrm{e}}_{\theta}$

F

$F(x), G(x), H(x)$

g

H

h

I

$\left.\begin{array}{l}I_{n+1 / 2}, K_{n+1 / 2}, I_{1 / 2}, \\ I_{-1 / 2}, I_{3 / 2}, I_{-3 / 2}\end{array}\right\}$

$J_{n+1 / 2}, J^{J}-(n+1 / 2)$

j

$\mathrm{K}, \mathrm{K}_{\mathrm{I}}, \mathrm{K}_{\mathrm{II}}$

$k, k_{I}, k_{\text {II }}$

M

$\mathrm{m}, \mathrm{n}$

$\overrightarrow{\mathrm{n}}$

P

$P_{n}^{1}(u), Q_{n}^{1}(u)$

$\mathrm{R}$

$\mathrm{R}^{\mathrm{P}}$

$\mathrm{R}_{1}$

r

t
One-half the distance between two coaxial coils

Electric intensity

Elliptic integrals of the second kind

Unit vectors in direction indicated

Force

Specified functions of $\mathrm{x}$

Gravitational constant

Magnetic - field intensity

Height of liquid-metal column

Current

Modified Bessel functions

Electric current density

Bessel functions

$(-1)^{1 / 2}$

Elliptic integrals of the first kind

Elliptic integral moduli

Magnetic dipole moment

Undefined powers of $R$

Unit normal vector

Power

Associated Legendre functions

Sphere radius; specified function of $r$

Specified function or $r$

Coil radius

Coordinate direction

Time 
u

$\mathrm{V}$

V

$v^{2 x}$

W

$\mathrm{x}$

$\mathrm{y}, \mathrm{y}_{\mathrm{I}}, \mathrm{y}_{\mathrm{II}}$

z
$\operatorname{Cos} \theta$

Arbitrary vector; scalar potential

Function of skin depth, sphere radius, and imaginary number

Complex conjugate of above quantity

Weight

Ratio of skin depth to sphere radius

Reduced axial distances

Coordinate direction 


\section{FOOTNOTES AND REFERENCES}

*Work performed under the auspices of the U.S. Atomic Energy Commission.

Presented at the Seventh Annual Vacuum Metallurgy Conference held

June 29 through July 1, 1964 in New York City and sponsored by the Vacuum Metallurgy Division of the American Vacuum Society.

1. NRC Equipment Corporation, 160 Charlemont Street, Newton 61, Massachusetts。

2. Vacuum Industries, Inc., 34 Linden Street, Somerville 43, Massachusetts.

3. Lepel High Frequency Laboratories, Inc., 55th Street and 37th Avenue, Woodside 77, L.I., New York, New York.

4. Tocco Division, The Ohio Crankshaft Company, 3800 Harvard Ave.,

Cleveland 5, Ohio.

5. Melting Metals in Mid.-Air。 Life (June 16, 1952), p. 49.

6. A. E. El-Mehairy, S. Y. Shiraishi, and R. G. Ward, Measurement of the Densities of Liquid Metals by a Levitated Drop Technique, J. Metals (Trans. Met. Soc. AIME) 16, 107 (1964).

7. R. T. Begley, G. Comenetz, P.A. Flinn, and J. W. Salatka, Vacuum Levitation Melting, Rev. Sci. Instr. 30, 38 (1959).

8. R. F. Bunshah (Metallurgy Department, Lawrence Radiation Laboratory, Livermore, California), private communication.

9. G. Comenetz and J.W. Salatka, Ten-Gram Levitation-Melted Ingots, J. Electrochem. Soc. 105, 673 (1958).

10. G.W. Toop and F.D. Richardson, Reactions Between Gases and Levitated Metals, J. Metals (Trans. Met. Soc. AIME) 16, 106 (1964). 
11. A.E. Jenkins, The Physico-Chemical Applications of Electromagnetic Levitation, Proceedings of the XVIII International Congress of Pure and Applied Chemistry (Montreal, Quebec, 1961), page 218; Temperature Control Improves Levitation, Chem. Eng. News 39, 38 (Aug. 21, 1961).

12. L. S. Baker, N.A. Warner, and A. E. Jenkins, Kinetics of Decarburization of Liquid Iron in an Oxidizing Atmosphere Using the Levitation Technique, J. Metals (Trans. Met.Soc. AIME) 16, 106 (1964).

13. A. Robertson and A. J. Rostron, Reaction of Molten Aluminum and of Aluminum-20\% Uranium Alloy with Water Vapor, J. Metals (Trans. Met. Soc, AIME) 16, 107 (1964)。

14. E.T. Turkdogan, The Theory of Enhancement of Diffusion-Limited Vaporization Rates by a Convection-Condensation Process: Part I -Theoretica1, J. Metals (Trans, Met. Soc. AIME) 16, 106 (1964).

15. E. T. Turkdogan and K. C. Mills, The Theory of Enhancement of Diffusion-Limited Vaporization Rates by a Convection-Condensation Process: Part II - Experimental, J. Metals, (Trans. Met. Soc. A.IME) 16,107 (1964).

16. K. C. Mills and K. Kinoshita, A Thermodynamic Study of the Iron-Nickel System Using Electromagnetic Levitation, J. Metals (Trans. Soc. Met, AIME) 16, 107 (1964)。

17. William R. Smythe, Static and Dynamic Electricity, (McGraw-Hill Book Company, Inc. New York, 1950), page 396.

18. J. Van Blade1, Electromagnetic Fields, IMcGraw-Hill Book Company, Inc., New York, 1964), page 274. 
19. E.C. Okress, D. M. Wroughton, G. Comenetz, P. H. Brace, and J. C. R. Kelly, Electromagnetic Levitation of Solid and Molten Metals, J. Appl. Phys. 23, 545, 1413 (1952).

20. W. J. Hulsey, The Design and Performance of Levitation Melting Coils, AEC Research and Development Report Y-1413, Apri1 26, 1963 (unpublished). Union Carbide Nuclear Company, Y-12 Plant, Oak Ridge, Tennessee.

21. B. Harris, and A. E. Jenkins, Controlled Atmosphere Levitation System, J. Sci. Inst. 36,238 (1959).

22. Colin J. Smithells, Metals Reference Book, (Butterworth and Co. , Ltd., Washington, D.C., 1962)。

23. R. B. Stewart and V.J. Johnson, A Compendium of the Properties of Materials at Low Temperature (Phase II), National Bureau of Standards, Boulder, Colorado. WADD Technical Report 60-56 Part IV, December 1961 (unpublished).

24. D. H. Polonis, R. G. Butters, and J. Gordon Parr, Some Techniques for Melting Reactive Metals, Research (London) 7, 273 (1954).

25. D. H. Polonis, R. G. Butters, and J. Gordon Parr, Levitation Melting Titanium and Titanium Alloys, Research (London) 7, S10 (1954).

26. L. R. Weisberg, Levitation Melting of $\mathrm{Ga}$, In, Au, and Sb, Rev. Sci. Inst. 30,135 (1959).

27. D. M. Wroughton, E.C. Okress, P.H. Brace, G. Comenetz, and J.C.R. Kelly, A Technique for Eliminating Crucibles in Heating and Melting of Metals, J. Electrochem. Soc. 97, 205 (1952)。

28. F.W. Bell Inc., 1356 Norton Avenue, Columbus 12, Ohio.

29. S. A. Colgate, H. P. Furth, and F.O. Halliday, Hydromagnetic Equilibrium Experiments with Liquid and Solid Sodium, Rev. Mod. Phys. 32, 744 (1960)。 
30. C. D. Hodgman, Handbook of Chemistry and Physics, (Chemical Rubber Publishing Co., Cleveland, Ohio, 1954), page 1989.

31. N. A. Lange, Handbook of Chemistry, (McGraw-Hill Book Company, Inc., New York, 1961), page 100.

32. S. W. Mayer, Calculation of Metal Surface Tensions. Ionic-Salt and Monatomic Models for Liquid Metals, J. Chem. Phys. 35, 1513 (1961).

33. J.W. Taylor, An Estimation of Some Unknown Surface Tensions for Metals, Atomic Energy Research Establishment, Harwell, Berks, England Report AERE-M/TN-24 (1954); Metallurgia 50, 161 (1954)。

34. S. W. Strauss, The Surface Tensions of Liquid Metals at their Melting Point, Nuc1. Sci. Eng。 8, 362 (1960).

35. S. W. Strauss, An Empirical Surface Tension-Temperature Relation for Liquid Metals, Reactor Science and Technology 15, 28 (1961).

36. Leo Brewer (Department of Chemistry and Inorganic Materials Research Division, Lawrence Radiation Laboratory, Berkeley, California), private communication.

37. The Arnold Engineering Company, Marengo, Mllinois.

38. P. G. Simpson, Induction Heating, (McGraw-Hill Book Company, Inc., New York, 1960), page 270.

39. D. R. Grafham, Now the Gate Turn Off Switch Speeds up D-C Switching, Electronics 37, 64 (March 23, 1964).

40. A. A. Fogel, Melting of Laboratory Specimens of Metals in Vacuum or an Inert Gas by Levitation Technique, Izv. Akad. Nauk SSSR, OTN, Metall. i Topl。2, 24 (1958); Henry Brutcher Technical Translation No. 4702, P. O. Box 157, Altadena, California.

41. Richard B. Adler, Lan Jen Chu, and Robert M. Fano, Electromagnetic Energy Transmission and Radiation, (John Wiley \& Sons, Inc., New York, 1960), page 15. 
42. Parry Moon and Domina Eberle Spencer, Foundations of Electrodynamics, (D. Van Norstrand Company, Inc。, Princeton, New Jersey, 1960), Page 118.

43. Max Abraham and Richard Becker, The Classical Theory of Electricity and Magnetism, Second Edition (Blackie \& Son Limited, London, 1950), page 90.

44. Michiyusu Takagi (Central Research Laboratory, Tokyo Shibaura Electric Co., Ltd, , 1 Komukai Toshiba-Cho, Kawasaki-Shi, Kanagawa-Ken, Japan), private communication. 
This report was prepared as an account of Government sponsored work. Neither the United States, nor the Commission, nor any person acting on behalf of the Commission:

A. Makes any warranty or representation, expressed or implied, with respect to the accuracy, completeness, or usefulness of the information contained in this report, or that the use of any information, apparatus, method, or process disclosed in this report may not infringe privately owned rights; or

B. Assumes any liabilities with respect to the use of, or for damages resulting from the use of any information, apparatus, method, or process disclosed in this report.

As used in the above, "person acting on behalf of the Commission" includes any employee or contractor of the Commission, or employee of such contractor, to the extent that such employee or contractor of the Commission, or employee of such contractor prepares, disseminates, or provides access to, any information pursuant to his employment or contract with the Commission, or his employment with such contractor. 